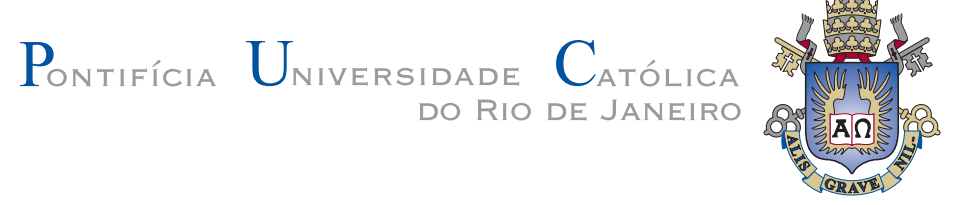

Jennifer Alexandra Mendez Rangel

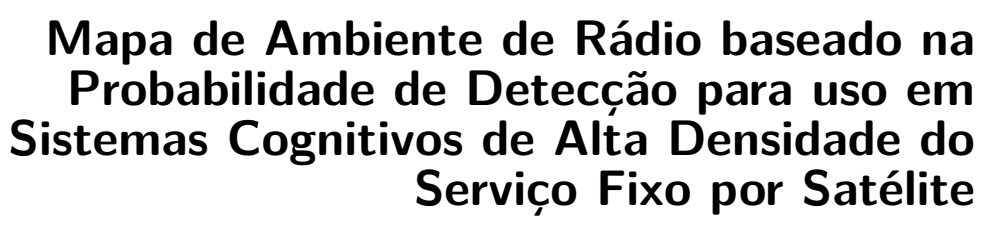

Tese de Doutorado

Tese apresentada como requisito parcial para obtenção do grau de Doutor pelo Programa de Pós-graduação em Engenharia Elétrica da PUC-Rio.

Orientador: Prof. José Mauro Pedro Fortes 


\title{
Mapa de Ambiente de Rádio baseado na Probabilidade de Deteccão para uso em Sistemas Cognitivos de Alta Densidade do Serviço Fixo por Satélite
}

Tese apresentada como requisito parcial para obtenção do grau de Doutor pelo Programa de Pós-graduação em Engenharia Elétrica da PUC-Rio. Aprovada pela Comissão Examinadora abaixo assinada.

\author{
Prof. José Mauro Pedro Fortes \\ Orientador \\ Departamento de Engenharia Elétrica - PUC-Rio \\ Prof. Raimundo Sampaio Neto \\ Pontifícia Universidade Católica do Rio de Janeiro - PUC-Rio \\ Prof. Paulo Roberto Rosa Lopes Nunes \\ Instituto Militar de Engenharia - IME \\ Prof. Pedro Henrique Gouvêa Coelho \\ Universidade do Estado do Rio de Janeiro - UERJ \\ Prof. Weiler Alves Finamore \\ Universidade Federal de Juiz de Fora - UFJF \\ Dr. Mauro Vieira de Lima \\ Instituto Nacional de Metrologia, Qualidade e Tecnologia - \\ INMETRO \\ Prof. Márcio da Silveira Carvalho \\ Coordenador Setorial do Centro Técnico Científico - PUC-Rio
}


Todos os direitos reservados. É proibida a reprodução total ou parcial do trabalho sem autorização da universidade, do autor e do orientador.

\section{Jennifer Alexandra Mendez Rangel}

Graduou-se em Engenharia de Telecomunicações na Universidad de San Buenaventura Bogotá, Colômbia. Fez mestrado no Departamento de Engenharia Elétrica da Pontifícia Universidade Católica do Rio de Janeiro, especializando-se na área de Sistemas de Comunicações.

Ficha Catalográfica

Mendez Rangel, Jennifer Alexandra

Mapa de Ambiente de Rádio baseado na Probabilidade de Detecção para uso em Sistemas Cognitivos de Alta Densidade do Serviço Fixo por Satélite / Jennifer Alexandra Mendez Rangel; orientador: José Mauro Pedro Fortes. - 2018.

108 f.: il. color. ; $30 \mathrm{~cm}$

Tese (doutorado) - Pontifícia Universidade Católica do Rio de Janeiro, Departamento de Engenharia Elétrica , 2018.

Inclui bibliografia

1. Engenharia Elétrica - Teses. 2. Mapa de Ambiente de Rádio;. 3. Comunicações por Satélite;. 4. HDFSS;. 5. Rádio Cognitivo;. I. Pedro Fortes, José Mauro. II. Pontifícia Universidade Católica do Rio de Janeiro. Departamento de Engenharia Elétrica. III. Título.

CDD: 621.3 
Amiga; incondicional, apasionada por tu profesión, con alma compasiva, defensora de la causa justa, amante de los aminales, profesante de amor infinito a tu familia. Quiero que sepas que fue $y$ siempre será un orgullo haberte conocido. 


\section{Agradecimentos}

A Deus e sua sábia vontade, por tudo o que eu sou, por meu passado, meu presente e meu futuro.

A minha família, sem dúvida, que tem me oferecido sempre seu infinito amor. Obrigada especial ao meu pai Lucas, minha mãe Alicia e minhas irmãs Catherine e Paula por serem a minha grande motivação, porque tudo foi obtido como uma vitória conjunta, resultado de todos os ensinamentos e do apoio que vocês têm me irradiado ao longo dos anos.

Ao amor da minha vida Mauricio, seu amor e suporte durante todo este tempo foram imprescindíveis para completar esta travessia.

A meu orientador e excelente professor José Mauro P. Fortes, por acreditar no meu trabalho e nas minhas habilidades, orientando-me de forma brilhante, por que cada minuto que trabalhei com ele, aprendi muito.

A PUC-Rio, ao seu corpo docente, por abrir-me as suas portas e deixarme crescer academicamente, concedendo-me uma excelente formação da mais alta qualidade.

A todas as pessoas que encontrei no caminho, entre eles compatriotas, colegas e amigos do CETUC, pela ajuda, pelos conselhos e pela amizade brindada.

Da mesma forma agradeço o apoio financeiro provido pelo $\mathrm{CNPq}$, oferecendo grandes oportunidades aos profissionais que desejam especializar-se e qualificar-se na formação de nível superior. 


\section{Resumo}

Mendez Rangel, Jennifer Alexandra; Pedro Fortes, José Mauro. Mapa de Ambiente de Rádio baseado na Probabilidade de Detecção para uso em Sistemas Cognitivos de Alta Densidade do Serviço Fixo por Satélite. Rio de Janeiro, 2018. 108p. Tese de Doutorado - Departamento de Engenharia Elétrica , Pontifícia Universidade Católica do Rio de Janeiro.

Este trabalho analisa um problema específico envolvendo o compartilhamento de frequências entre o Serviço Fixo Terrestre (FS) e uma aplicação de alta densidade do Serviço Fixo por Satélite (HDFSS). Este problema, identificado pela primeira vez durante as discussões sobre sistemas de alta densidade ocorridas durante a Conferência Mundial de Radiocomunicações da União Internacional de Telecomunicações de 2003 tem, desde então, sido objeto de diversos estudos. No cenário geral considerado neste trabalho, sistemas do FS e do HDFSS compartilham a mesma faixa de frequências em uma mesma região geográfica. Os sistemas do FS operam como usuários primários e os sistemas HDFSS operam, utilizando técnicas de Rádio Cognitivo, como usuários secundários. Para facilitar a convivência entre os dois serviços, o uso de um Mapa de Ambiente de Rádio (REM - Radio Environment Map) é considerado. Este tipo de mapa, produzido por um Centro de Base de Dados (Data Base Center) a partir de informações recebidas dos usuários secundários, indica em que partes da região geográfica considerada um determinado canal está disponível para uso pelos usuários secundários. Neste trabalho, um novo método de geração de REM é proposto e avaliado. No desenvolvimento do método é utilizada uma modelagem original que considera uma densidade espectral de potência genérica para os sinais transmitidos pelos usuários primários. Além disso, métricas adequadas para avaliar a qualidade de REMs e o desempenho dos métodos de geração são definidas e propostas. Estas métricas são utilizadas numa análise comparativa de desempenho envolvendo o método de geração de REM proposto e um método de geração existente. A análise é feita com base nos resultados obtidos para cenários específicos que, diferentemente dos cenários utilizados em outros trabalhos, considera a existência de múltiplos enlaces do FS na região de interesse.

\section{Palavras-chave}

Mapa de Ambiente de Rádio; Comunicações por Satélite; HDFSS; Rádio Cognitivo; 


\section{Abstract}

Mendez Rangel, Jennifer Alexandra; Pedro Fortes, José Mauro (Advisor). A Detection Probability based Radio Environment Map for use in Cognitive High Density Fixed Satellite Systems. Rio de Janeiro, 2018. 108p. Tese de Doutorado - Departamento de Engenharia Elétrica, Pontifícia Universidade Católica do Rio de Janeiro.

This work analyzes a specific problem involving the frequency sharing between the Fixed Service (FS) and a High Density application in the Fixed Satellite Service (HDFSS). This problem was first identified through discussions on high density systems held at the 2003 World Radiocommunication Conference and, since then, it has been the object of several studies. This study considers a general scenario involving a geographical area where FS systems shares its operating frequency band with HDFSS systems. The FS systems operate as primary users and the HDFSS systems operate as secondary users using Cognitive Radio techniques. To facilitate the frequency sharing between these two systems, the use of a Radio Environment Map (REM) is considered. This kind of map, produced by a Data Base Center (DBC), is based on the collaboration data received from all secondary users and indicates those locations (inside the considered geographical area) where a given frequency channel is available for use by secondary users. In this work, a new REM generation method is proposed and evaluated. In developing the method, an original mathematical modeling, that considers a generic power spectrum density for the primary users transmissions, is used. Furthermore, appropriate metrics are defined and proposed aiming to assess the REM quality and the REM generation method performance. These metrics are used in a comparative performance analysis involving the proposed REM generation method and an existing generation method. This analysis is based on results obtained for specific scenarios that, unlike those evaluated in other studies, consider the existence of multiple FS links in the geographical area of interest.

\section{Keywords}

Radio Environment Map; Satellite Communications; HDFSS; Cognitive Radio; 


\section{Sumário}

1 Introdução $\quad 16$

2 Descrição do problema $\quad 19$

2.1 Sistemas de Alta densidade do Serviço Fixo por Satélite 19

2.2 Compartilhamento de frequências entre aplicações HDFSS e o FS 22

3 Modelagem Matemática $\quad 25$

3.1 Densidade de potência interferente produzida pelas transmissões de usuários primários $\quad 26$

3.2 Teste de hipóteses binário $\quad 27$

3.3 Caracterização do ruído de observação 29

3.4 Caracterização do sinal produzido pelas emissões dos usuários primários 32

3.4.1 Sinal Determinístico 32

3.4.2 Processo Estocástico gaussiano ESA 33

3.5 Modelagem do vetor de observações 35

3.6 Critério de detecção de Neyman-Pearson 36

3.7 Métricas de desempenho do detector 39

3.7.1 Funções densidade de probabilidade condicionais da variável de decisão 41

3.7.2 Probabilidade de falso alarme e probabilidade de detecção 44

3.8 Estimador da potência média da envoltória complexa do sinal do usuário primário $\quad 45$

3.8.1 Estimação a partir do vetor de observações $\mathbf{r} \quad 46$

3.8.2 Estimação a partir da observação da variável de decisão $v \quad 46$

3.9 Caso particular: PE gaussiano ESA com DEP plana na faixa 47

3.9.1 Variável de decisão 48

3.9.2 Funções densidade de probabilidade condicionais da variável de decisão 49

3.9.3 Probabilidade de falso alarme e probabilidade de detecção 51

3.9.4 Estimador da potência média da envoltória complexa do sinal do usuário primário $\quad 52$

4 Mapas de Ambiente de Rádio $\quad \mathbf{5 4}$

4.1 Métodos de Geração dos Mapas de Ambiente de Rádio 54

4.1.1 Método 0 54

4.1.2 Método $1 \quad 56$

4.2 Qualidade dos Mapas de Ambiente de Rádio 59

5 Resultados Numéricos $\quad 61$

5.1 Parâmetros técnicos dos sistemas do Serviço Fixo Terrestre (usuários primários) 62

5.2 Parâmetros técnicos dos enlaces HDFSS (usuários secundários) 64

5.3 Esquema de Simulação 66

5.4 Desempenho dos métodos de geração de REM 68

5.4.1 Cenário 1: apenas um usuário primário operando na região de interesse 69

5.4.2 Cenário 2: três usuários primários operando na região de interesse 80 
5.4.3 Comentários adicionais relativos aos resultados obtidos nos cenários 1 e 2

6 Conclusões $\quad 93$

$\begin{array}{lll}7 & \text { Referências bibliográficas } & 96\end{array}$

A Soma de variáveis aleatórias com função densidade de probabilidade exponencial e estatisticamente independentes

A.1 Procedimento 1: utilizando o método dos resíduos 101

A.2 Procedimento 2: utilizando um caso particular da série convergente proposta em [1]

B Estimadores utilizados pelos USs e no DBC

B.1 Estimador com base na observação da variável de decisão $v$ (sinais de UPs com densidade espectral de potência genérica)

B.2 Estimador com base na observação da variável de decisão $v$ (sinais de UPs com densidade espectral de potência plana)

B.3 Utilização do estimador de potência para sinais com DEP plana em situações onde a DEP dos sinais é não plana

B.3.1 Estimação feita nos usuários secundários

B.3.2 Estimação feita no Centro de Base de Dados 


\section{Lista de figuras}

Figura 2.1 Cenário de interesse FS/HDFSS 22

Figura 3.1 Cenário de referência para o cálculo da densidade de potência interferente $I_{r} \quad 26$

Figura 3.2 Densidade espectral de potência do ruído filtrado $n_{f}(t) \quad 30$

Figura 3.3 Densidade espectral de potência da envoltória complexa do ruído filtrado $\tilde{n}_{f}(t) \quad 30$

Figura 3.4 Função autocorrelação $R_{\tilde{n}_{f}}(\tau)$

Figura 3.5 Valores do limiar a ser utilizado no teste de hipóteses em função da potência média $\beta$.

Figura 4.1 Diagrama em blocos do Método_0 de geração do REM 55

Figura 4.2 Distribuição geográfica marcada dos usuários secundários

Figura 4.3 Exemplo de resultados para o Método_0 e $N_{\mathrm{US}}=900 \quad 56$

Figura 4.4 Diagrama em blocos do Método_1 de geração do REM 56

Figura 4.5 Distribuição geográfica marcada dos USs cooperadores 58

Figura 4.6 Exemplo de resultados para o Método_1 e $N_{\text {US }}=900 \quad 58$

Figura 4.7 Mapas REM de referência e mapa REM gerado $\quad 59$

Figura 5.1 Enlaces do Serviço Fixo Terrestre e enlaces do HDFSS em um cenário de compartilhamento de frequências

Figura 5.2 Diagrama de radiação da antena transmissora do FS

Figura 5.3 Módulo e fase da função autocorrelação $R_{\tilde{s}}(\tau)(a=$ $2,4725 \times 10^{5} \mathrm{~Hz}$ e $\left.f_{d}=0,2 \mathrm{MHz}\right)$.

Figura 5.4 Distribuição dos USs na região de interesse para $\lambda_{p p p}=$ 1 pontos $/ \mathrm{km}^{2}$

Figura 5.5 Diagrama de radiação da antena receptora do terminal HDFSS

Figura 5.6 Esquema de simulação utilizado na obtenção das métricas de desempenho dos métodos de geração de REM avaliados

Figura 5.7 Localização do UP na região de interesse

Figura 5.8 Densidade de potência interferente agregada produzida pelo transmissor FS na saída da antena receptora do terminal HDFSS em função da sua localização.

Figura 5.9 Mapa de Ambiente de Rádio de referência $\left(S_{d_{r e f}}\right)$ para um nível de $I_{\max }=-139 \mathrm{~dB}(\mathrm{~W} / \mathrm{MHz})$.

Figura 5.10 Pares $\left(T_{f a}, T_{d}\right)$ obtidos para o Cenário 1, Caso 1 com $\lambda_{p p p}=0,5$ pontos $/ \mathrm{km}^{2}$

Figura 5.11 Pares $\left(T_{f a}, T_{d}\right)$ obtidos para o Cenário 1, Caso 1 com $\lambda_{p p p}=1$ pontos $/ \mathrm{km}^{2}$

Figura 5.12 Pares $\left(T_{f a}, T_{d}\right)$ obtidos para o Cenário 1, Caso 1 com $\lambda_{p p p}=1,5$ pontos $/ \mathrm{km}^{2}$

Figura 5.13 Desempenho dos métodos de geração de REM: efeito da densidade de USs, Cenário 1, Caso 1 
Figura 5.14 Pares $\left(T_{f a}, T_{d}\right)$ obtidos para o Cenário 1, Caso 2 com $\lambda_{p p p}=0,5$ pontos $/ \mathrm{km}^{2}$

Figura 5.15 Pares $\left(T_{f a}, T_{d}\right)$ obtidos para o Cenário 1, Caso $2 \mathrm{com}$ $\lambda_{\text {ppp }}=1$ pontos $/ \mathrm{km}^{2}$

Figura 5.16 Pares $\left(T_{f a}, T_{d}\right)$ obtidos para o Cenário 1, Caso 2 com $\lambda_{p p p}=1,5$ pontos $/ \mathrm{km}^{2}$

Figura 5.17 Desempenho dos métodos de geração de REM: efeito da densidade de USs, Cenário 1, Caso 2

Figura 5.18 Pares $\left(T_{f a}, T_{d}\right)$ obtidos para o Cenário 1, Caso 3 com $\lambda_{p p p}=0,5$ pontos $/ \mathrm{km}^{2}$

Figura 5.19 Pares $\left(T_{f a}, T_{d}\right)$ obtidos para o Cenário 1, Caso 3 com $\lambda_{p p p}=1$ pontos $/ \mathrm{km}^{2}$

Figura 5.20 Pares $\left(T_{f a}, T_{d}\right)$ obtidos para o Cenário 1, Caso $3 \mathrm{com}$ $\lambda_{p p p}=1,5$ pontos $/ \mathrm{km}^{2}$

Figura 5.21 Desempenho dos métodos de geração de REM: comparação dos casos 1 e 3, Cenário 1

Figura 5.22 Localização dos três UPs na região de interesse

Figura 5.23 Densidade de potência interferente agregada produzida pelos transmissores FS na saída da antena receptora do terminal HDFSS em função da sua localização.

Figura 5.24 Mapa de Ambiente de Rádio de referência $\left(S_{d_{r e f}}\right)$ para um nível de $I_{\max }=-139 \mathrm{~dB}(\mathrm{~W} / \mathrm{MHz})$.

Figura 5.25 Pares $\left(T_{f a}, T_{d}\right)$ obtidos para o Cenário 2, Caso 1 com $\lambda_{p p p}=0,5$ pontos $/ \mathrm{km}^{2}$

Figura 5.26 Pares $\left(T_{f a}, T_{d}\right)$ obtidos para o Cenário 2, Caso 1 com $\lambda_{p p p}=1$ pontos $/ \mathrm{km}^{2}$

Figura 5.27 Pares $\left(T_{f a}, T_{d}\right)$ obtidos para o Cenário 2, Caso 1 com $\lambda_{p p p}=1,5$ pontos $/ \mathrm{km}^{2}$

Figura 5.28 Desempenho dos métodos de geração de REM: efeito da densidade de USs, Cenário 2, Caso 1

Figura 5.29 Pares $\left(T_{f a}, T_{d}\right)$ obtidos para o Cenário 2, Caso 2 com $\lambda_{p p p}=0,5$ pontos $/ \mathrm{km}^{2}$

Figura 5.30 Pares $\left(T_{f a}, T_{d}\right)$ obtidos para o Cenário 2, Caso $2 \mathrm{com}$ $\lambda_{p p p}=1$ pontos $/ \mathrm{km}^{2}$

Figura 5.31 Pares $\left(T_{f a}, T_{d}\right)$ obtidos para o Cenário 2, Caso 2 com $\lambda_{p p p}=1,5$ pontos $/ \mathrm{km}^{2}$

Figura 5.32 Desempenho dos métodos de geração de REM: efeito da densidade de USs, Cenário 2, Caso 2

Figura 5.33 Pares $\left(T_{f a}, T_{d}\right)$ obtidos para o Cenário 2, Caso $3 \mathrm{com}$ $\lambda_{p p p}=0,5$ pontos $/ \mathrm{km}^{2}$

Figura 5.34 Pares $\left(T_{f a}, T_{d}\right)$ obtidos para o Cenário 2, Caso 3 com $\lambda_{p p p}=1$ pontos $/ \mathrm{km}^{2}$

Figura 5.35 Pares $\left(T_{f a}, T_{d}\right)$ obtidos para o Cenário 2, Caso $3 \mathrm{com}$ $\lambda_{p p p}=1,5$ pontos $/ \mathrm{km}^{2}$

Figura 5.36 Desempenho dos métodos de geração de REM: comparação dos casos 1 e 3, Cenário 2

Figura 5.37 Desempenho dos métodos de geração de REM: comparação dos cenários 1 e 2, Caso 1 
Figura 5.38 Desempenho dos métodos de geração de REM: comparação dos cenários 1 e 2, Caso 2

Figura 5.39 Desempenho dos métodos de geração de REM: comparação dos cenários 1 e 2, Caso 3 


\section{Lista de tabelas}

Tabela 2.1 Faixas de frequências identificadas para a operação de sistemas HDFSS

Tabela 5.1 Coordenadas do transmissor e receptor do enlace FS considerado.

Tabela 5.2 Coordenadas dos transmissores e receptores dos três enlace FS considerados.

Tabela B.1 Média aritmética dos valores de $\Delta \bar{T}_{d}$ e $\Delta \bar{T}_{f a}$ para cada um dos casos e cenários abordados na Seção 5.4

Tabela B.2 Estatísticas do erro de estimação para vários valores de $\beta=B \mathrm{~dB}(\mathrm{~W} / \mathrm{MHz})$ 


\title{
Lista de siglas
}

\author{
DBC - Data Base Center \\ DEP - Densidade Espectral de Potência \\ ESA - Estacionário no Sentido Amplo \\ FS - Fixed Service \\ FSS - Fixed Satellite Service \\ GEO - Geosynchronous Equatorial Orbit \\ HDFSS - High Density application in the Fixed Satellite Service \\ ML - Maximum Likelihood \\ PE - Processo Estocástico \\ PPP - Processo Pontual de Poisson \\ RC - Rádio Cognitivo \\ REM - Radio Environment Map \\ ROC - Receiver Operating Characteristics \\ $\mathrm{RR}$ - Regulamento de Radiocomunicações \\ UIT - União Internacional de Telecomunicações \\ UP - Usuário Primário \\ UPs - Usuários Primários \\ US - Usuário Secundário \\ USs - Usuários Secundários \\ WRC - World Radiocommunication Conference
}




\section{Notação}
$\mathbf{A}^{\mathcal{H}}$
Operador Hermitiano da matriz A
I
Matriz identidade
$\mathcal{F}^{-1} \quad$ Transformada inversa de Fourier
$\operatorname{diag}\left(\left\{a_{i}\right\}_{1}^{M}\right) \quad$ Matriz diagonal cuja diagonal contém os elementos $\left\{a_{i}, i=1, \ldots, M\right\}$ $\mathbb{R}$
O conjunto dos números reais 


\section{1 \\ Introdução}

Nos últimos anos, a demanda por recursos do espectro de radiofrequências em sistemas de comunicações sem fio tem apresentado um crescimento exponencial que está relacionado ao aumento massivo de novos usuários e ao surgimento de aplicações que consomem grandes bandas de frequência (aplicações de banda larga). O espectro de radiofrequências disponível para atender esses requisitos, que já é limitado, está se tornando escasso. Além disso, devido à atual alocação fixa e à segmentação, várias porções do espectro licenciado não são utilizadas durante períodos de tempo significativos. [2-5]. Uma possível solução para a escassez de espectro é permitir que usuários não licenciados, ou seja Usuários Secundários (USs), obtenham acesso dinâmico ao espectro disponível em certos canais de frequência. O Rádio Cognitivo (RC) apresenta-se como a tecnologia que possibilita esta alternativa, avaliando constantemente o estado destes canais e fazendo uso deles quando se encontram desocupados.

O foco de pesquisa em Rádio Cognitivo, que tem sido amplamente desenvolvido para redes terrestres, começou recentemente a ser estudado visando aplicações na área de comunicações por satélite [6-10]. Estes estudos têm identificado cenários adequados para um possível uso de rádios cognitivos em comunicações por satélite. O cenário envolvendo uma região geográfica na qual enlaces do Sistema Fixo Terrestre (FS - Fixed Service), operando como serviço primário, e sistemas de alta densidade do Serviço Fixo por Satélite (HDFSS - High Density application in the Fixed Satellite Service), operado como serviço secundário, compartilham a mesma faixa de frequências, por exemplo, tem recebido grande atenção [7,11-13].

As técnicas sugeridas para aplicação em cenários envolvendo rádios cognitivos possuem usualmente duas etapas, com diferentes finalidades. Na primeira etapa (denominada spectrum awareness), a ocupação do espectro é avaliada visando identificar canais de frequência disponíveis, em instantes e localizações geográficas especificas, que poderiam ser utilizados de forma compartilhada pelos USs. Esta etapa provê informação sobre os níveis de interferência em cada um destes canais para estabelecer se os usuários secundários estão sujeitos a interferência prejudicial. A segunda etapa (denominada spectrum exploitation) [14], está relacionada ao aproveitamento dos canais de frequência 
previamente identificados como disponíveis e utiliza técnicas cognitivas para mitigar, a um nível aceitável, as interferências previamente identificadas na etapa de spectrum awareness. Esta mitigação pode ser obtida, por exemplo, utilizando-se técnicas de formação de feixe (beam-forming) [15] e de realocação dos recursos de frequência disponíveis [16]. Este trabalho considera o cenário particular FS/HDFSS e concentra-se na etapa de spectrum awareness, onde usualmente são adotadas duas técnicas: (i) sensoriamento de espectro spectrum sensing e (ii) uso de um Centro de Base de Dados (DBC - Data Base Center).

O sensoriamento de espectro é uma técnica para adquirir conhecimento sobre a utilização do espectro do usuário primário em uma localização geográfica especifica. As técnicas mais comuns são a Detecção de energia, Detecção por Cicloestacionariedade e Detecção por Filtro Casado [7,17,18]. Quando se trata do cenário FS/HDFSS os terminais do sistema HDFSS podem potencialmente empregar técnicas de sensoriamento de espectro para obter informação sobre a interferência produzida por enlaces do serviço FS em sua localização geográfica especifica, identificando os canais de frequência disponíveis para eles usarem [13]. Assumindo que a sensibilidade da técnica de sensoriamento de espectro é suficientemente alta para detectar o nível máximo de interferência que o terminal HDFSS pode tolerar, a decisão (identificação de canais disponíveis de frequência) pode ser baseada unicamente na observação do sensoriamento de espectro feita pelo próprio terminal [12].

O uso de uma base de dados aparece como outra opção para o spectrum awareness. Sob esta abordagem as informações sobre as características técnicas e operacionais de enlaces do FS potencialmente interferentes na faixa de frequência de interesse (localizados em uma área geográfica específica) são armazenadas em uma base de dados. A informação detalhada sobre os canais de frequência, larguras de banda, localizações dos terminais FS, diagramas de radiação e de polarização, ganhos de antena e potência de transmissão também podem ser incluídos nesta base [13]. Toda esta informação é centralizada em um Centro de Base de Dados (DBC -Data Base Center) que, utilizando modelos de propagação conhecidos, calcula a interferência produzida pelos transmissores FS em qualquer ponto da região geográfica de interesse. Desta forma os terminais HDFSS podem consultar o DBC a fim de verificar os canais de frequência que não estão expostos à interferência prejudicial do FS $[12,19]$. Embora eficiente, esta técnica requer informação detalhada e confiável sobre os enlaces do FS, que pode não estar disponível devido a informações imprecisas ou incompletas ou mesmo devido a restrições de confidencialidade. Neste caso, o DBC teria que estimar a interferência do FS 
com base nas informações fornecidas individualmente pelo sensoriamento de espectro dos terminais HDFSS. Esta técnica combinada de spectrum awareness pode fornecer uma decisão mais precisa e confiável sobre o uso do espectro.

Note que a informação vinda apenas de um US pode não abordar o problema de caracterização do espectro com a confiabilidade necessária e, por esta razão, a cooperação entre diferentes terminais cognitivos localizados na área de interesse pode ser necessária (sensoriamento de espectro cooperativo cooperative spectrum sensing). A ideia é que os terminais do HDFSS possam executar o sensoriamento de espectro de forma individual, enviando ao DBC informação que pode ser usada por uma técnica de sensoriamento cooperativo. Neste contexto, o trabalho em [11] propõe uma técnica em que cada US executa o sensoriamento de espectro (por meio da técnica de detecção de energia) e decide se existe ou não sinal interferente de UP no canal de frequência analisado, enviando ao DBC a informação da sua decisão juntamente com sua localização. Com base nos dados recebidos de todos os USs cooperadores, o DBC produz uma estimativa da distribuição espacial da interferência gerada pelos UPs, que é utilizada para obter um mapa binário indicando as regiões nas quais um US pode operar. Este mapa binário é usualmente conhecido como mapa de ambiente de rádio (REM - Radio Environment Map), e é utilizado pelo DBC para autorizar a operação de novos terminais HDFSS.

Conforme já mencionado, o cenário considerado neste trabalho, que diz respeito ao compartilhamento de frequências entre uma aplicação do HDFSS operando na direção espaço-Terra e enlaces do Serviço Fixo Terrestre operando na mesma área geográfica, já havia sido identificado pela Resolução 143 da Conferência Mundial de Comunicações de 2003 [20]. Devido às características especificas do sistema HDFSS (eg. grande número de terminais e implantação ubíqua), que dificultam a coordenação entre os sistemas, é considerada uma situação em que os enlaces do FS operam como serviço primário e a aplicação do HDFSS como serviço secundário. No Capítulo 2, o problema de compartilhamento de frequências é identificado. Expressões matemáticas aplicáveis à modelagem matemática do problema são desenvolvidas no Capítulo 3. No Capítulo 4, são especificados dois métodos de geração de REM: o método proposto em [11] e um método original, proposto neste trabalho. Além disso, são definidas duas métricas adequadas para a avaliação de métodos ne geração de REMs. No Capítulo 5, uma avaliação dos dois métodos de geração de REM considerados é feita com base em resultados numéricos obtidos pela aplicação destes métodos a cenários específicos. Finalmente no Capítulo 6 são apresentadas as conclusões resultantes do desenvolvimento deste trabalho. 


\section{2 \\ Descrição do problema}

Neste capítulo é descrito o problema do compartilhamento de frequências entre aplicações de alta densidade do Serviço Fixo por Satélite (HDFSS) e sistemas do Serviço Fixo Terrestre (FS). Inicialmente, na Seção 2.1, é apresentado um breve histórico identificando, entre outras coisas, recomendações e resoluções do Setor de Radiocomunicações da União Internacional de Telecomunicações (ITU-R) que abordam a utilização de sistemas HDFSS. São indicadas as características específicas dos sistemas HDFSS (e.g. terminais de baixo custo, alta densidade de terminais e ubiquidade) que dificultam o compartilhamento de frequências entre eles e sistemas de outros serviços. Na Seção 2.2 é descrito um cenário particular de estudo em que uma aplicação do HDFSS compartilha, em caráter secundário, uma faixa de frequências com sistemas do Serviço Fixo Terrestre, estes operando em caráter primário. Neste cenário, considera-se a utilização de técnicas de Rádio Cognitivo na mitigação da interferência causada pelos transmissores FS nos terminais HDFSS.

\section{1}

\section{Sistemas de Alta densidade do Serviço Fixo por Satélite}

A demanda por serviços de banda larga tem aumentado muito ultimamente, tanto para fins residenciais como comerciais, sendo refletida, por exemplo, no crescimento do acesso em banda larga via satélite [21]. Os serviços de banda larga via satélite oferecem uma alternativa bastante viável aos sistemas de banda larga terrestres, com aplicações não só nas áreas urbanas quanto também nas áreas suburbanas e rurais. Considerando-se que este tipo de sistema requer um tempo maior de implementação em comparação com sistemas terrestres, garantias regulamentares que evidenciem a viabilidade da implantação de um sistemas do Serviço Fixo por Satélite (FSS - Fixed Satellite Service) são essenciais. Atualmente, diferentes redes do Serviço Fixo por Satélite planejam operar na Banda K (sub-bandas Ku e Ka) e muitas dessas redes têm como finalidade fornecer, em partes desta banda, serviços envolvendo estações terrenas de pequeno porte (terminais) e implantadas de maneira ubíqua (HDFSS - High Density application in the Fixed Satellite Service). Assim, o Setor de Radiocomunicações da União Internacional de Telecomunicações (UIT-R) questionou 
quais seriam as características técnicas dos terminais do FSS implantados com uma alta densidade (Questão UIT-R 266/4 [22]), visando definir recomendações contendo diretrizes para facilitar sua operação e ajudar na realização de estudos de compartilhamento de frequências entre este tipo de redes e outros serviços, especificando os parâmetros técnicos a serem utilizados nestes estudos.

Como resultado desta questão, foram realizados estudos que produziram, inicialmente, a Recomendação UIT-R S.1594 [23], que define os níveis máximos de emissão dos terminais de uma aplicação de alta densidade no Serviço Fixo por Satélite (HDFSS - High Density application in the Fixed Satellite Service) na direção da órbita de satélites geoestacionários (GEO) e de requisitos associados. Uma outra recomendação resultou da Questão ITU-R 266/4. A Recomendação UIT S.1783 [24], que define as características dos sistemas via satélite que podem ser caracterizados como uma aplicação de alta densidade no Serviço Fixo por Satélite (HDFSS), indicando algumas de suas características gerais, entre elas,

- A implementação dos terminais do sistema HDFSS é flexível, rápida é ubíqua;

- Os terminais HDFSS são implantados na área de serviço em um número muito grande, resultando numa alta densidade geográfica;

- Os terminais HDFSS são de baixo custo e empregam antenas pequenas (e.g. diâmetro menor a 1.8 metros [23]);

- A distribuição geográfica dos terminais pode ser feita em nível urbano, suburbano e rural;

- As redes HDFSS fornecem acesso a uma ampla variedade de aplicações de telecomunicações de banda larga, incluindo a Internet e;

- Aplicações de alta densidade no Serviço Fixo por Satélite podem fazer uso de satélites localizados na orbita de satélites geoestacionários (GEO) ou em órbita de satélites não geoestacionários (não-GEO).

Na Conferência Mundial de Radiocomunicações de 2003 (WRC'03 World Radiocommunication Conference) foram feitas modificações no Artigo 5 do Regulamento de Radiocomunicações da UIT (RR) [25], que define a alocação do espectro de radiofrequências aos diversos serviços. Foi adicionada, no artigo, a Nota No. 5.516B que identifica porções das faixas de frequência inicialmente alocadas para o Serviço Fixo por Satélite para o uso por sistemas HDFSS. Na Tabela 2.1 são identificadas estas faixas de frequência.

De acordo com o Artigo 5 do RR [25], é possível identificar que algumas das faixas de frequência da Tabela 2.1, são compartilhadas, em caráter 
Tabela 2.1: Faixas de frequências identificadas para a operação de sistemas HDFSS

\begin{tabular}{|c|c|c|c|}
\hline Direção & Região 1 & Região 2 & Região 3 \\
\hline \multirow{7}{*}{ Espaço-terra } & 17.3-17.7GHz & \multirow{3}{*}{ 18.3-19.3 GHz } & \\
\hline & $39.5-40 \mathrm{GHz}$ & & \\
\hline & $47.5-47.9 \mathrm{GHz}$ & & \\
\hline & $48.2-48.54 \mathrm{GHz}$ & \multirow{2}{*}{$40.5-42 \mathrm{GHz}$} & \\
\hline & $48.2-48.54 \mathrm{GHz}$ & & \\
\hline & \multicolumn{3}{|c|}{$19.7-20.2 \mathrm{GHz}$} \\
\hline & \multicolumn{3}{|c|}{$40-40.5 \mathrm{GHz}$} \\
\hline \multirow{6}{*}{ Terra-espaço } & \multirow{4}{*}{$27.5-27.82 \mathrm{GHz}$} & $28.35-28.45 \mathrm{GHz}$ & \\
\hline & & $29.25-29.46 \mathrm{GHz}$ & \\
\hline & & $48.2-50.2 \mathrm{GHz}$ & \\
\hline & & $28.94-29.1$ & $\mathrm{~Hz}$ \\
\hline & \multicolumn{3}{|c|}{$28.45-28.94 \mathrm{GHz}$} \\
\hline & \multicolumn{3}{|c|}{ 29.46-30 GHz } \\
\hline
\end{tabular}

primário com outros serviços. Isto significa que a identificação das faixas de frequências para o uso por sistemas HDFSS, não impede o uso dessas faixas por outros serviços ou por outras aplicações FSS. Além disso, esta identificação não estabelece prioridade entre os diferentes serviços, que podem utilizar estas faixas fazendo-se necessária coordenação entre os diferentes sistemas. Entretanto, dadas as características de alta densidade e ubiquidade do HDFSS, este tipo de coordenação torna-se impraticável.

Conforme identificado na Resolução 143 da UIT [20] aprovada na WRC'03, o compartilhamento de frequências entre terminais do HDFSS e serviços terrestres (fixos, móveis ou de radiodifusão) na mesma área geográfica é um processo difícil, e pode, em alguns casos, ser facilitado através da implementação de possíveis técnicas de mitigação de interferência sem a necessidade de coordenação individual entre todos os terminais do HDFSS e as estações do serviço terrestre.

Por este motivo, embora as faixas de frequência da Tabela 2.1 estejam alocadas ao FSS em caráter primário, é aventada a possibilidade da utilização das faixas por aplicações HDFSS, em caráter secundário, como uma forma de mitigação dos problemas. Um caso específico, abordado na próxima seção diz respeito ao compartilhamento de frequências entre aplicações HDFSS e o Serviço Fixo Terrestre (FS). 


\section{2}

\section{Compartilhamento de frequências entre aplicações HDFSS e o FS}

O presente trabalho abordada uma situação particular do problema de compartilhamento do HDFSS com outros serviços, identificado na Resolução 143 [20]. Mais especificamente, é analisado o compartilhamento de frequências entre terminais de um sistema HDFSS e sistemas do serviço terrestre, operando na mesma área geográfica. É considerado um cenário envolvendo uma região geográfica onde o FS e uma aplicação do HDFSS operando na direção espaçoterra compartilham a mesma faixa de frequências. Conforme mencionado anteriormente, as características específicas do HDFSS (e.g. grande número de terminais e implantação ubíqua) sugerem que a coordenação individual entre todos os terminais do HDFSS e as estações do FS pode ser um processo difícil e longo. Para superar esse problema, embora essas faixas de frequência sejam alocadas para o FS e o FSS em caráter primário, é razoável considerar uma situação onde os transmissores do FS operam como Usuários Primários (UPs) e os terminais do HDFSS como Usuários Secundários (USs).

Nesta situação, conforme ilustrado na Figura 2.1, são identificados dois percursos de interferência. O primeiro percurso está relacionado à interferência que as transmissões do satélite podem gerar no receptor do enlace FS. Neste caso, a proteção do FS é dada pelos limites máximos de densidade de fluxo de potência que a transmissão de um satélite pode produzir na superfície da Terra, constantes do Artigo 21 do Regulamento de Radiocomunicações [25] da UIT. O segundo percurso identificado, corresponde à interferência que as emissões do transmissor do FS podem gerar no terminal do HDFSS que, dado o carácter secundário da vitima, não pode ser contestada.

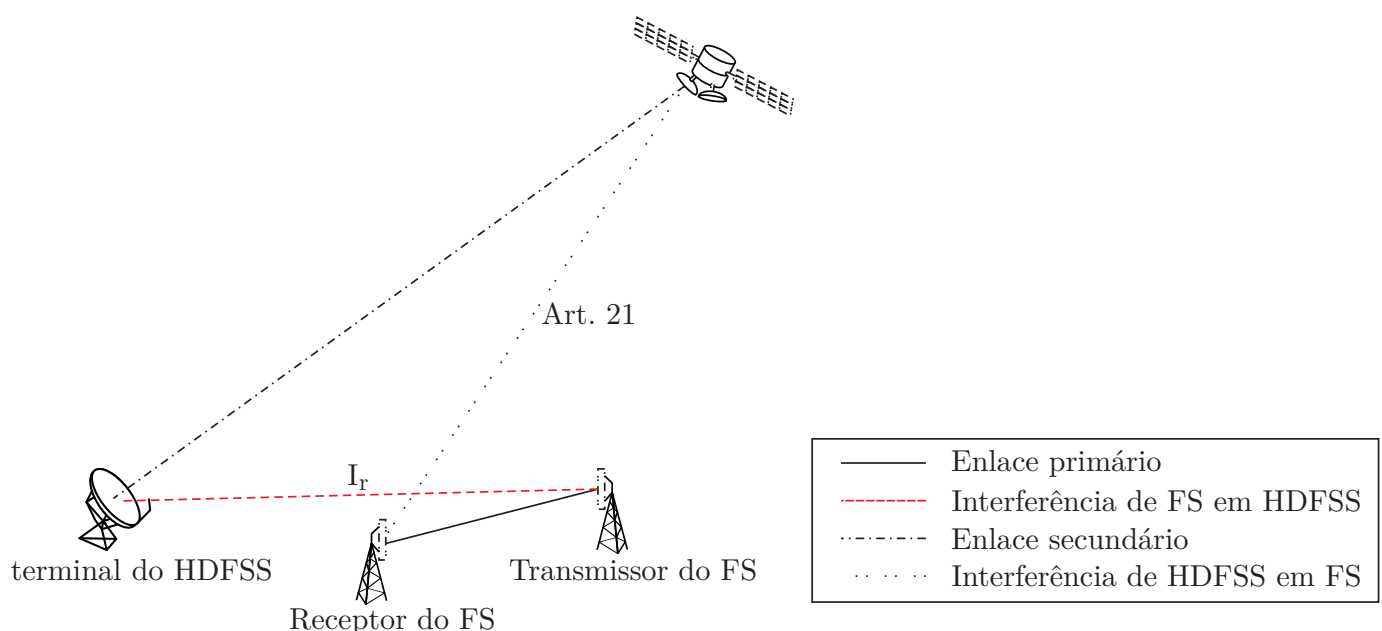

Figura 2.1: Cenário de interesse FS/HDFSS

Nesta situação o uso de técnicas de Rádio Cognitivo no sistema HDFSS 
poderia ser muito útil. Sob estas técnicas, por exemplo, antes de utilizar um determinado canal de frequência para transmitir informação (no sentido espaço-Terra) em direção a um terminal específico, o sistema HDFSS deve verificar se o canal candidato está livre de interferências prejudiciais produzidas pelos transmissores FS.

Com relação ao cenário FS/HDFSS, os terminais do sistema HDFSS podem potencialmente empregar técnicas de sensoriamento de espectro para obter informação sobre a potencial interferência produzida por enlaces do serviço FS em sua localização geográfica, identificando os canais de frequência disponíveis [13]. Assumindo que a sensibilidade da técnica de sensoriamento de espectro utilizada pelo terminal HDFSS é o suficientemente alta para detectar se a interferência produzida pelos enlaces FS é maior ou menor que o nível máximo de interferência que o terminal HDFSS pode tolerar, a decisão sobre a disponibilidade do canal poderia ser feita com base apenas na observação feita [12] por este terminal.

Note, porém, que a informação vinda de apenas um US pode não abordar o problema de sensoriamento do espectro com a confiabilidade necessária, devido, por exemplo, ao seu nível de ruído térmico. Para contornar este problema, é usual se utilizar a cooperação entre os vários usuários secundários operando na região de interesse. Esta técnica é conhecida como sensoriamento cooperativo de espectro (denominada cooperative spectrum sensing).

A ideia então é incorporar Rádios Cognitivos aos terminais do HDFSS para que cada um deles execute o sensoriamento de espectro na sua localização geográfica e envie, para um Centro de Base de Dados (DBC - Data Base Center), além das informações sobre sua localização, as informações sobre parâmetros relacionados à presença de transmissões de usuários primários na área de serviço do HDFSS. Com base nos dados recebidos, o DBC produz a estimativa de uma distribuição espacial que reflete a interferência gerada pelos UPs que operam na área de serviço do HDFSS. Com base nesta distribuição espacial, o DBC produz um mapa binário que indica as regiões da área de serviço HDFSS nas quais os USs podem operar, mesmo na presença da interferência produzida pelas transmissões dos UPs. Este mapa é denominado o Mapa de Ambiente de Rádio (REM) e é utilizado pelo DBC para autorizar ou não a operação de terminais HDFSS em um determinado canal. Note que é necessária a geração de vários REM, um para cada canal de recepção dos terminais HDFSS. Assim, antes de utilizar um determinado canal de frequência para transmitir informação (no sentido espaço-Terra) em direção a um terminal específico, o sistema HDFSS deve verificar se o canal candidato está livre de interferências prejudiciais produzidas pelos transmissores FS. 
O problema do sensoriamento cooperativo de espectro é abordado neste trabalho. Além de propor um novo método de geração de REM, o trabalho define métricas que podem ser utilizadas na determinação do desempenho e na análise comparativa de desempenho de métodos de geração de REM, feita através de curvas do tipo ROC (Receiver Operating Characteristics). 


\section{3 \\ Modelagem Matemática}

Neste capitulo são deduzidas todas as expressões matemáticas aplicáveis à modelagem matemática do problema em análise. Com base na geometria associada ao cenário de interferência HDFSS/FS, na Seção 3.1 é apresentada a expressão matemática que fornece o nível de densidade de potência interferente que as transmissões de um enlace FS produzem na saída da antena receptora de um terminal do serviço HDFSS. Na Seção 3.2, o problema de sensoriamento de espectro, executado por cada US, é modelado como um teste de hipóteses binário e a caracterização dos sinais envolvidos neste teste, ou seja do ruído de observação e do sinal produzido pelas emissões dos usuários primários, é apresentada nas seções 3.3 e 3.4, respectivamente.

Considerando que o sinal $s(t)$, produzido pelas transmissões de usuários primários, é modelado por um processo estocástico gaussiano estacionário no sentido amplo com densidade espectral de potência genérica, na Seção 3.5 é apresentada a caracterização estatística do vetor observado, formado por amostras da envoltória complexa do sinal atingindo a antena de recepção do terminal HDFSS. Devido à falta de informação sobre as probabilidades a priori no teste de hipóteses, este trabalho considera, como critério de detecção, o de Neyman-Pearson, definido na Seção 3.6. O desempenho deste critério é avaliado pelas métricas: probabilidade de falso alarme e probabilidade de detecção. Expressões para estas duas métricas são determinadas na Seção 3.7. O cálculo destas métricas pode requerer o conhecimento da potência media da envoltória complexa do sinal do UP. Nestes casos, optou-se por utilizar o estimador de máxima verossimilhança deste parâmetro, cujas expressões são desenvolvidas na Seção 3.8 .

Finalmente, na Seção 3.9, os resultados obtidos nas seções anteriores deste capítulo são particularizadas para o caso em que o sinal $s(t)$ produzido pelas transmissões dos usuários primários, é caracterizado por um processo estocástico gaussiano, estacionário no sentido amplo com densidade espectral de potência plana na faixa. 


\section{1}

\section{Densidade de potência interferente produzida pelas transmissões de usuários primários}

Nesta seção é apresentada a expressão matemática que descreve o nível de potência interferente, que a transmissão de um enlace FS, pode causar na saída da antena receptora de um terminal do serviço HDFSS.

Na Figura 3.1 é ilustrado o cenário de referência, e a partir da geometria associada é definida a expressão matemática que permite calcular o nível de densidade de potência interferente produzida pelas transmissões de um enlace do Serviço Fixo Terrestre. Primeiramente é considerado que um satélite na orbita geoestacionária transmite no lance de descida em direção a um terminal HDFSS localizado na mesma área geográfica onde encontra-se implantado um enlace do Serviço Fixo Terrestre.

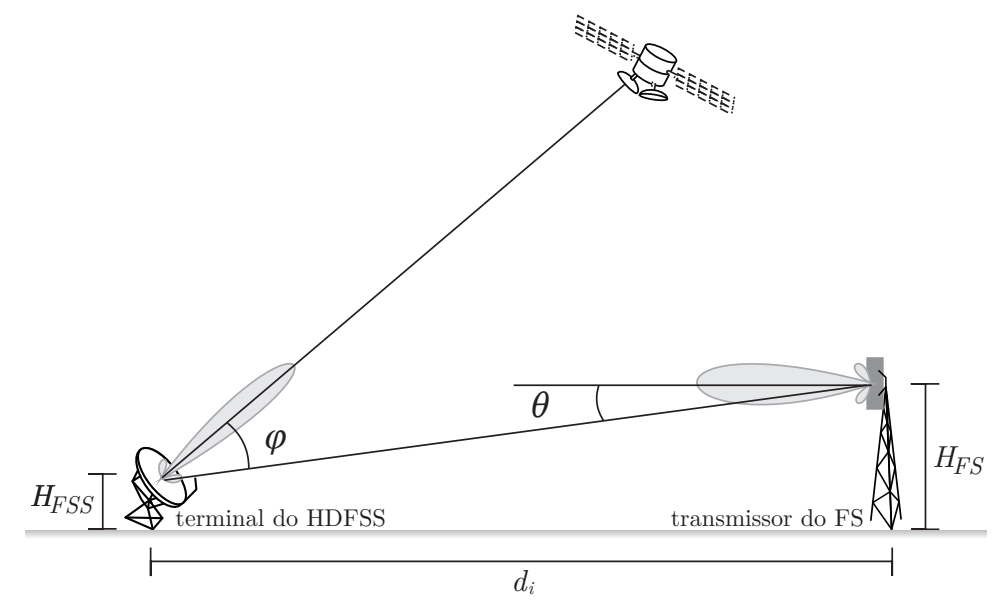

Figura 3.1: Cenário de referência para o cálculo da densidade de potência interferente $I_{r}$

Na Figura 3.1, $H_{F S}$ e $H_{F S S}$, expressas em metros, representam as alturas do transmissor do Serviço Fixo Terrestre e do terminal do HDFSS respectivamente. O valor $\theta$ representa o ângulo entre a direção do lóbulo principal do transmissor do FS e a direção do terminal HDFSS a partir da posição do transmissor do FS, da mesma forma $\phi$ representa o ângulo entre a direção de apontamento do terminal HDFSS e a direção do transmissor do FS a partir da posição do terminal.

Considerando-se duas antenas no espaço livre, como no cenário da Figura 3.1, separadas por uma distância $d_{i}$, a densidade de fluxo de potência interferente $d p i_{F S S}$, expressa em $\mathrm{W} /\left(\mathrm{MHz} \mathrm{m}^{2}\right)$, que atinge a antena receptora do terminal do HDFSS, separada uma distância $d_{i}$ da antena transmissora do FS, é dada por

$$
d p i_{F S S}=\frac{p_{F S}}{4 \pi d_{i}^{2}} g_{F S}(\theta)
$$


onde, $p_{F S}$ representa a densidade de potência de transmissão (W/MHz) e $g_{F S}(\theta)$ representa o ganho da antena do transmissor do FS na direção do terminal do HDFSS.

A densidade de potência interferente nos terminais da antena receptora do FSS é então dada por

$$
i_{r}=d p i_{F S S} A_{e f}(\phi)
$$

onde $A_{e f}(\phi)$ representa a área efetiva da antena receptora do FSS na direção corresponednte a $\phi$ graus fora do eixo. Esta área efetiva, dada por [26]

$$
A_{e f}(\phi)=\frac{\ell^{2}}{4 \pi} g_{F S S}(\phi)
$$

com $g_{F S S}(\phi)$ denotando o ganho da antena receptora do FSS numa direção $\phi$ graus fora do eixo e $\ell$ denotando o comprimento de onda associado ao canal de frequência em estudo. Assim, considerando (3-3) e (3-1), o nível de densidade de potência interferente nos terminais da antena receptora do HDFSS é dado por

$$
i_{r}=p_{F S} g_{F S}(\theta) g_{F S S}(\phi)\left(\frac{\ell}{4 \pi d_{i}}\right)^{2}
$$

ou ainda, quando expresso em dB (W/MHz), é dado por

$$
I_{r}=P_{F S_{d B}}+G_{F S_{d B}}(\theta)+G_{F S S_{d B}}(\phi)+20 \log _{10}\left(\frac{\ell}{4 \pi d_{i}}\right)
$$

\section{2}

\section{Teste de hipóteses binário}

O sensoriamento de espectro em Rádios Cognitivos visa obter conhecimento da utilização que fazem os usuários primários de certa faixa de frequência. Uma vez que os usuários do Rádio Cognitivo (usuários secundários) devem decidir sobre a presença ou ausência de usuários primários, com base na observação do sinal recebido na entrada de sua antena. Assim o problema em questão pode ser definido como um teste de hipóteses binário onde o sinal observado é dado por

$$
r(t)=\left\{\begin{array}{ll}
n(t) & : H_{0} \\
s(t)+n(t) & : H_{1}
\end{array} \quad ; \quad 0<t \leq T\right.
$$

com as hipóteses $H_{0}$ e $H_{1}$ representando, respectivamente, a ausência e a presença de sinal produzido pela transmissão de usuários primários. Note que em (3-6), o processo estocástico $n(t)$, representa o ruído de observação presente no sistema.

Usualmente nos sistemas de comunicações via rádio, o sinal da fonte de 
informação modula uma portadora de alta frequência antes da sua transmissão, ou seja, o sinal transmitido é caracterizado por um processo estocástico passafaixa com por exemplo frequência central $f_{0}$.

Conforme já mencionado, o objetivo do usuário secundário é detectar a presença do usuário primário a partir da observação do sinal recebido na entrada de sua antena. Este sinal passa inicialmente por um filtro passa-faixa de modo a minimizar os efeitos do ruído e das interferências externas fora da faixa.

Este filtro é usualmente modelado [27-31], por um filtro passa-faixa ideal com frequência central $f_{0}$ e largura de banda $W$. Assim, após o filtro de recepção, o sinal observado escreve-se

$$
r_{f}(t)=\left\{\begin{array}{ll}
n_{f}(t) & : H_{0} \\
s(t)+n_{f}(t) & : H_{1}
\end{array} \quad ; \quad 0<t \leq T\right.
$$

onde $n_{f}(t)$ representa o ruído de observação filtrado. Note que em (3-7), considerou-se que a largura $W$ do filtro é maior que a banda do sinal $s(t)$.

Uma maneira conveniente de se tratar sinais passa-faixa é através do conceito de envoltória complexa (definido em relação a uma dada frequência de referencia $f_{r}$ ) [32]. No caso de $f_{r}$ ser igual à frequência central $f_{0}$ do sinal passa-faixa, os sinais passa-faixa são associados a sinais complexos de baixa frequência (espectro em torno da origem), com a metade da largura de banda.

Assim, em termos de envoltória complexa, (3-7) pode ser escrita como

$$
\tilde{r}_{f}(t)= \begin{cases}\tilde{n}_{f}(t) & : H_{0} \\ \tilde{s}(t)+\tilde{n}_{f}(t) & : H_{1}\end{cases}
$$

onde $\tilde{r}_{f}(t), \tilde{s}(t)$ e $\tilde{n}_{f}(t)$ representam as envoltórias complexas em relação à frequência central $f_{0}$, do sinal passa-faixa.

Ao invés de se trabalhar com a observação analógica $\left\{\tilde{r}_{f}(t), \quad 0<t \leq T\right\}$ é comum em muitos casos $[28,29,31,33,34]$, utilizar, na deteç̧ão de $\tilde{s}(t)$, amostras do sinal observado, tomadas à taxa de Nyquist. Neste caso

$$
r_{i}=\tilde{r}_{f}(i \Delta) ; \quad i=0, \ldots, M-1
$$

onde

$$
\begin{aligned}
& \Delta=1 / W \\
& M=\left\lfloor\frac{T}{\Delta}\right\rfloor=\lfloor T W\rfloor
\end{aligned}
$$

Note que $\left\{r_{i}, \quad i=0, \ldots, M-1\right\}$ são variáveis aleatórias complexas que podem ser representadas pelo vetor aleatório complexo n-dimensional

$$
\mathbf{r}=\left(r_{0}, r_{1}, \ldots, r_{M-1}\right)^{\mathrm{T}}
$$

Assim a observação em (3-8) é equivalente à observação do vetor $\mathbf{r}$ dado por 


$$
\mathbf{r}= \begin{cases}\mathbf{n} & : H_{0} \\ \mathbf{s}+\mathbf{n} & : H_{1}\end{cases}
$$

onde

$$
\mathbf{n}=\left(\tilde{n}_{f}(0), \tilde{n}_{f}(\Delta), \ldots, \tilde{n}_{f}((M-1) \Delta)\right)^{\mathrm{T}}
$$

e

$$
\mathbf{s}=(\tilde{s}(0), \tilde{s}(\Delta), \ldots, \tilde{s}((M-1) \Delta))^{\mathrm{T}}
$$

\section{3}

\section{Caracterização do ruído de observação}

Neste trabalho considera-se que o ruído de observação $n(t)$ presente no sistema corresponde ao ruído térmico existente em sistemas eletrônicos. Normalmente este tipo de ruído é modelado por um Processo Estocástico (PE) Estacionário no Sentido Amplo (ESA) gaussiano com Densidade Espectral de Potência (DEP) plana (ruído branco) dada por

$$
S_{n}(f)=\frac{N_{0}}{2}
$$

e consequentemente com função autocorrelação dada por

$$
R_{n}(\tau)=\frac{N_{0}}{2} \delta(\tau)
$$

Assim, o ruído filtrado pelo filtro passa-faixa ideal de recepção, $n_{f}(t)$, é também um processo estocástico gaussiano de média nula e com densidade espectral de potência dada por

$$
S_{n_{f}}(f)=\frac{N_{0}}{2}\left[\operatorname{ret}_{W}\left(f-f_{0}\right)+\operatorname{ret}_{W}\left(f+f_{0}\right)\right]
$$

onde $\operatorname{ret}_{W}$ representa a função retângulo definida por

$$
\operatorname{ret}_{W}(f)= \begin{cases}1 & |f| \leq \frac{W}{2} \\ 0 & |f|>\frac{W}{2}\end{cases}
$$

e (3-17) é ilustrada na Figura 3.2. Portanto a densidade espectral de potência da envoltória complexa do ruído $n_{f}(t)$ em relação a frequência $f_{0}$ é dada por [?]

$$
S_{\tilde{n}_{f}}(f)=4 U\left(f+f_{0}\right) S_{n_{f}}\left(f+f_{0}\right)
$$

onde $U(f)$ é a função degrau dada por

$$
U(f)= \begin{cases}1 & f>0 \\ 1 / 2 & f=0 \\ 0 & f<0\end{cases}
$$

considerando-se (3-17), tem-se que a densidade espectral de potência da envoltória complexa $\tilde{n}_{f}(t)$ é dada por 


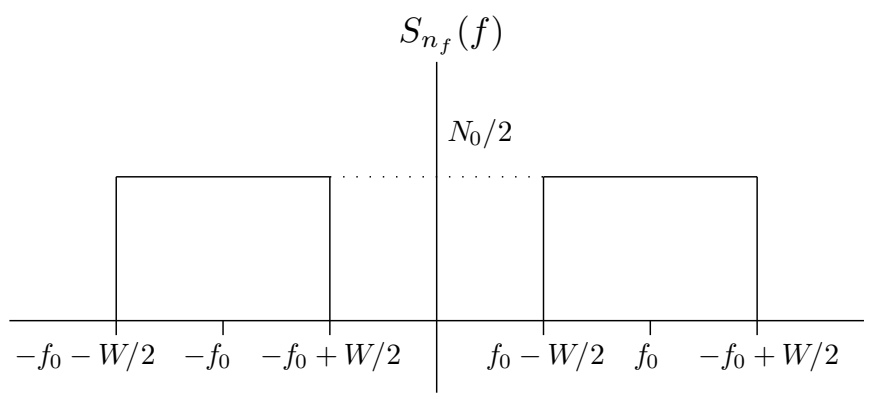

Figura 3.2: Densidade espectral de potência do ruído filtrado $n_{f}(t)$

$$
S_{\tilde{n}_{f}}(f)=2 N_{0} \operatorname{ret}_{W}(f)
$$

esta densidade é ilustrada na Figura 3.3, e em consequência a função autocorrelação de $\tilde{n}_{f}(t)$ é então dada por

$$
R_{\tilde{n}_{f}}(\tau)=2 N_{0} W \operatorname{sinc}(W \tau)
$$

com a função $\operatorname{sinc}(\cdot)$ definida por

$$
\operatorname{sinc}(x)=\left\{\begin{array}{lll}
1 & ; & x=0 \\
\frac{\operatorname{sen}(x \pi)}{x \pi} & ; & x \neq 0
\end{array}\right.
$$

A expressão (3-22) é ilustrada na Figura 3.4. A potência média da

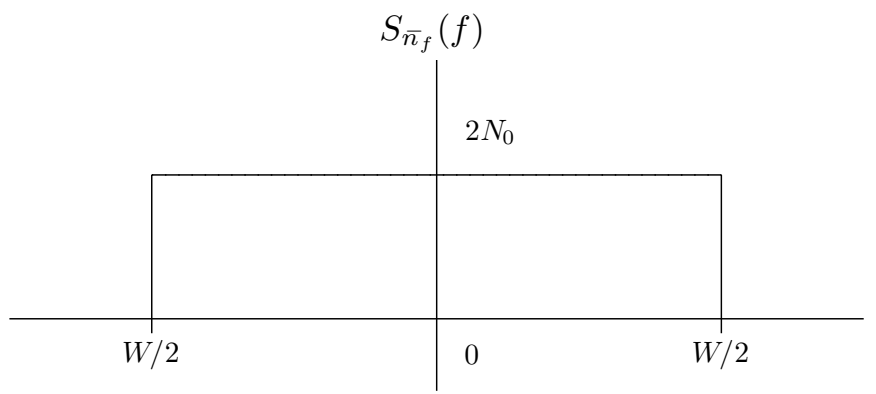

Figura 3.3: Densidade espectral de potência da envoltória complexa do ruído filtrado $\tilde{n}_{f}(t)$

envoltória complexa $\tilde{n}_{f}(t)$ é dada por

$$
P_{\tilde{n}_{f}}=\int_{-\infty}^{\infty} S_{\tilde{n}_{f}}(f) d f
$$

ou, como $\tilde{n}_{f}(t)$ é um processo estacionário no sentido amplo

$$
P_{\tilde{n}_{f}}=R_{\tilde{n}_{f}}(0)
$$

Assim, considerando-se (3-22) tem-se 


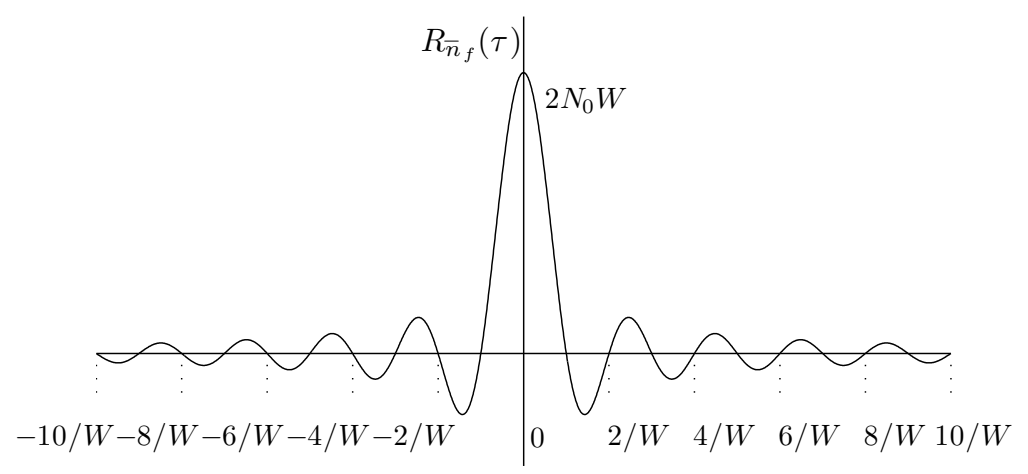

Figura 3.4: Função autocorrelação $R_{\tilde{n}_{f}}(\tau)$

$$
P_{\tilde{n}_{f}}=R_{\tilde{n}_{f}}(0)=2 N_{0} W
$$

a potência média da envoltória complexa $\tilde{n}_{f}(t)$ pode ainda ser escrita como

$$
P_{\tilde{n}_{f}}=2 \sigma_{n}^{2}
$$

com

$$
\sigma_{n}^{2}=N_{0} W
$$

Note que quando o processo $\tilde{n}_{f}(t)$ é amostrado à taxa Nyquist $(\Delta=$ $1 / W)$, a partir de (3-23) obtém-se amostras descorrelatadas, com média

$$
m_{n_{i}}=\mathrm{E}\left[\tilde{n}_{f}(i \Delta)\right]=0
$$

e variância

$$
\sigma_{n_{i}}^{2}=\mathrm{E}\left[\left(n_{i}-m_{n_{i}}\right)\left(n_{i}-m_{n_{i}}\right)^{*}\right]=\left[\left|n_{i}\right|^{2}\right]=2 N_{0} W=2 \sigma_{n}^{2}
$$

Assim o conjunto de amostras descorrelatadas pode ser representado por um vetor aleatório complexo definido por

$$
\mathbf{n}=\left(\tilde{n}_{f}(0), \tilde{n}_{f}(\Delta),,, \quad \tilde{n}_{f}((M-1) \Delta)\right)^{\mathrm{T}}
$$

com média

$$
\mathbf{m}_{\mathbf{n}}=\mathbf{0}
$$

e matriz covariância $\mathbf{K}_{\mathbf{n}}=\mathrm{E}\left[\mathbf{n} \mathbf{n}^{\mathcal{H}}\right]$, dada por

$$
\mathbf{K}_{\mathbf{n}}=\left[\begin{array}{cccc}
2 \sigma_{n}^{2} & 0 & \ldots & 0 \\
0 & 2 \sigma_{n}^{2} & \ldots & 0 \\
\vdots & \vdots & \ddots & \vdots \\
0 & 0 & \ldots & 2 \sigma_{n}^{2}
\end{array}\right]
$$

ou ainda

$$
\mathbf{K}_{\mathbf{n}}=2 \sigma_{n}^{2} \mathbf{I}
$$

Note que, como o processo estocástico $n(t)$ é gaussiano, ESA e de mé- 
dia nula, $n_{f}(t)$ é também gaussiano e ESA. Consequentemente a envoltória complexa do ruído filtrado $\tilde{n}_{f}(t)$, é um processo estocástico gaussiano complexo, ESA, de média nula e circularmente simétrico [35] (neste caso, $\mathrm{E}\left[\tilde{n}_{f}\left(t_{1}\right) \tilde{n}_{f}\left(t_{2}\right)\right]=0 \forall\left(t_{1}, t_{2}\right)$ ). Por conseguinte, as amostras do processo $\tilde{n}_{f}(t)$, formam um vetor $\mathbf{n}$ aleatório complexo gaussiano circularmente simétrico [36] $\left(\mathrm{E}\left[\mathbf{n} \mathbf{n}^{\mathrm{T}}\right]=0\right)$, com componentes estatisticamente independentes, ou seja o vetor aleatório complexo $\mathbf{n}$ é definido por uma função densidade de probabilidade gaussiana multivariada dada por [37]

$$
p_{\mathbf{n}}(\mathbf{N})=\frac{1}{\pi^{M} \operatorname{det} \mathbf{K}_{\mathbf{n}}} \exp \left(-\left(\mathbf{N}-\mathbf{m}_{\mathbf{n}}\right)^{\mathrm{H}} \mathbf{K}_{\mathbf{n}}^{-1}\left(\mathbf{N}-\mathbf{m}_{\mathbf{n}}\right)\right)
$$

ou ainda em notação compacta,

$$
\mathbf{n} \sim \mathcal{C N}\left(\mathbf{0}, 2 \sigma_{n}^{2} \mathbf{I}\right)
$$

\section{4}

\section{Caracterização do sinal produzido pelas emissões dos usuários primários}

Uma compilação dos vários trabalhos de pesquisa relacionados à modelagem do sinal de usuário primário em um ambiente Rádio Cognitivo sem desvanecimento mostrou que, na maioria dos trabalhos, o sinal produzido no receptor do US pela transmissão de um UP, $s(t)$ é modelado como um sinal determinístico [18, 29, 30,34,38,39], conforme desenvolvimento feito na Subseção 3.4.1. Alguns dos trabalhos caracterizam $s(t)$ como um processo estocástico ESA $[11,18,31,33,34,40-42]$, mas consideram o caso particular de densidade espectral de potência plana para o processo $s(t)$.

Neste trabalho é apresentado o desenvolvimento matemático que considera $s(t)$ como um processo estocástico gaussiano, estacionário no sentido amplo, com densidade espectral de potência genérica (ver Subseção 3.4.2).

\subsection{1}

\section{Sinal Determinístico}

Nesta subseção considera-se que o sinal produzido pela transmissão de usuários primários, $s(t)$, é caracterizado por um sinal real determinístico passafaixa com transformada de Fourier dada por

$$
S(f)=\mathcal{F}[s(t)]
$$

Considerando-se um filtro de recepção passa-faixa ideal com banda passante $W$, maior que a banda do sinal $s(t)$, tem-se na saída do filtro

$$
s(t)=\operatorname{Re}\left\{\tilde{s}(t) e^{j 2 \pi f_{0} t}\right\}
$$


onde $\tilde{s}(t)$ é a envoltória complexa do sinal com relação à frequência $f_{0}$ e sua transformada de Fourier é dada por [32]

$$
\tilde{S}(f)=\mathcal{F}[\tilde{s}(t)]=2 U\left(f+f_{0}\right) S\left(f+f_{0}\right)
$$

Amostras da envoltória complexa $\tilde{s}(t)$, tomadas a intervalos $\Delta=1 / W$, formam um vetor complexo determinístico dado por

$$
\mathbf{s}=(\tilde{s}(0), \tilde{s}(\Delta), \tilde{s}(2 \Delta), \ldots, \tilde{s}(i \Delta), \ldots, \tilde{s}((M-1) \Delta))^{\mathrm{T}}
$$

onde

$$
M=\left\lfloor\frac{T}{\Delta}\right\rfloor=\lfloor T W\rfloor
$$

onde $T$ é o período de amostragem.

\subsection{2}

\section{Processo Estocástico gaussiano ESA}

Nesta subseção considera-se que o sinal produzido pelas transmissões de usuários primários $s(t)$ é modelado por um processo estocástico passa-faixa (i.e. a densidade espectral de potência do processo é não desprezível somente em uma faixa de frequência de largura $W$ cuja frequência central é muito maior que $W / 2)$, gaussiano, ESA e de média nula $\left(m_{s}(t)=0\right)$, com função autocorrelação $R_{s}(\tau)$ e densidade espectral de potência $S_{s}(f)$.

O processo estocástico passa-faixa $s(t)$, pode ser representado por meio da sua envoltória complexa $\tilde{s}(t)$ em relação à uma frequência de referência $f_{0}$, ou seja,

$$
s(t)=\operatorname{Re}\left\{\tilde{s}(t) e^{-2 \pi f_{0} t}\right\}
$$

onde a envoltória complexa $\tilde{s}(t)$ é caracterizada por um processo estocástico complexo gaussiano, ESA, de média $m_{\tilde{s}}(t)=0$, com densidade espectral de potência dada por

$$
S_{\tilde{s}}(f)=4 U\left(f+f_{0}\right) S_{s}\left(f+f_{0}\right)
$$

onde $U(f)$ é a função degrau unitário definida em (3-20). A potência média da envoltória complexa $\tilde{s}(t)$ é dada por

$$
P_{\tilde{s}}=\int_{-\infty}^{\infty} S_{\tilde{s}}(f) d f=R_{\tilde{s}}(0)
$$

onde $R_{\tilde{s}}(\tau)=\mathrm{E}\left[\tilde{s}(t) \tilde{s}^{*}(t+\tau)\right]$ é a função autocorrelação do processo estocástico $\tilde{s}(t)$, dada por

$$
R_{\tilde{s}}(\tau)=\mathcal{F}^{-1}\left[S_{\tilde{s}}(f)\right]
$$

Note que a densidade espectral de potência da envoltória complexa $\tilde{s}(t)$ pode ser escrita como

$$
S_{\tilde{s}}(f)=\beta S_{\tilde{s}_{0}}(f)
$$

onde $S_{\tilde{s}_{0}}(f)$ corresponde à densidade espectral de potência da envoltória 
complexa normalizada $\tilde{s}_{0}(t)$ (potência unitária), ou seja,

$$
\int_{-\infty}^{\infty} S_{\tilde{s}_{0}}(f) d f=1
$$

Considerando-se (3-45) e (3-46), tem-se ainda,

$$
R_{\tilde{s}}(\tau)=\beta R_{\tilde{s}_{0}}(\tau)
$$

$\operatorname{com} R_{\tilde{s}_{0}}(\tau)$ denotando a função autocorrelação da envoltória complexa normalizada $\tilde{s}_{0}(t)$ (ouseja, $\left.R_{\tilde{s}_{0}}(0)=1\right)$.

Observe que a envoltória complexa $\tilde{s}(t)$ se relaciona à envoltória complexa normalizada $\tilde{s}_{0}(t)$ pela relação

$$
\tilde{s}(t)=\sqrt{\beta} \tilde{s}_{0}(t)
$$

Note, a partir de (3-44), (3-46) e (3-47), que a potência média da envoltória complexa $\tilde{s}(t)$ é dada por

$$
P_{\tilde{s}}=\beta
$$

Amostras do processo estocástico complexo $\tilde{s}(t)$, tomadas a intervalos $\Delta=1 / W$ (Taxa de Nyquist), constituem uma coleção de variáveis aleatórias complexas, conjuntamente gaussianas, que formam o vetor aleatório gaussiano complexo

$$
\mathbf{s}=(\tilde{s}(0), \tilde{s}(\Delta), \ldots, \tilde{s}((M-1) \Delta))^{\mathrm{T}}
$$

que, a partir da relação em (3-49), pode ser escrito como

$$
\mathbf{s}=\sqrt{\beta} \mathbf{s}_{0}
$$

onde

$$
\mathbf{s}_{0}=\left(\tilde{s}_{0}(0), \tilde{s}_{0}(\Delta), \ldots, \tilde{s}_{0}((M-1) \Delta)\right)^{\mathrm{T}}
$$

Observe que, como o processo estocástico $s(t)$ é gaussiano, ESA e de média nula, a envoltória complexa $\tilde{s}(t)$ é um processo estocástico gaussiano complexo ESA de média nula e circularmente simétrico [35] (neste caso, $\left.\mathrm{E}\left[\tilde{s}\left(t_{1}\right) \tilde{s}\left(t_{2}\right)\right]=0 \forall\left(t_{1}, t_{2}\right)\right)$. Assim, as amostras da envoltória complexa $\tilde{s}(t)$ formam um vetor aleatório s complexo gaussiano e circularmente simétrico [36], que tem uma função densidade de probabilidade dada por [37],

$$
p_{\mathbf{s}}(\mathbf{S})=\frac{1}{\pi^{M} \operatorname{det} \mathbf{K}_{\mathbf{s}}} \exp \left(-\left(\mathbf{S}-\mathbf{m}_{\mathbf{s}}\right)^{\mathrm{H}} \mathbf{K}_{\mathbf{s}}^{-1}\left(\mathbf{S}-\mathbf{m}_{\mathbf{s}}\right)\right)
$$

ou ainda, em notação compacta,

$$
\mathbf{s} \sim \mathcal{C N}\left(\mathbf{0}, \mathbf{K}_{\mathbf{s}}\right)
$$

Note que, em (3-54), o vetor média $\mathbf{m}_{\mathbf{s}}$ e a matriz covariância $\mathbf{K}_{\mathbf{s}}$ são dados respectivamente por 


$$
\mathbf{m}_{\mathbf{s}}=\mathrm{E}[\mathbf{s}]=\left(m_{\tilde{s}}(0), m_{\tilde{s}}(\Delta), \ldots, m_{\tilde{s}}((M-1) \Delta)\right)^{\mathrm{T}}=\mathbf{0}
$$

e

$$
\mathbf{K}_{\mathbf{s}}=\mathrm{E}\left[\mathbf{s ~ s}^{\mathcal{H}}\right]=\left[\begin{array}{cccc}
R_{\tilde{s}}(0) & R_{\tilde{s}}^{*}(\Delta) & \ldots & R_{\tilde{s}}^{*}((M-1) \Delta) \\
R_{\tilde{s}}(\Delta) & R_{\tilde{s}}(0) & \ldots & R_{\tilde{s}}^{*}((M-2) \Delta) \\
R_{\tilde{s}}(2 \Delta) & R_{\tilde{s}}(\Delta) & \ldots & R_{\tilde{s}}^{*}((M-3) \Delta) \\
\vdots & \vdots & \ddots & \vdots \\
R_{\tilde{s}}((M-1) \Delta) & R_{\tilde{s}}((M-2) \Delta) & \ldots & R_{\tilde{s}}(0)
\end{array}\right]
$$

ou ainda, considerando (3-48),

$$
\mathbf{K}_{\mathbf{s}}=\beta \mathbf{K}_{\mathbf{s}_{0}}
$$

com

$$
\mathbf{K}_{\mathbf{s}_{0}}=\left[\begin{array}{cccc}
R_{\tilde{s}_{0}}(0) & R_{\tilde{s}_{0}}^{*}(\Delta) & \ldots & R_{\tilde{s}_{0}}^{*}((M-1) \Delta) \\
R_{\tilde{s}_{0}}(\Delta) & R_{\tilde{s}_{0}}(0) & \ldots & R_{\tilde{s}_{0}}^{*}((M-2) \Delta) \\
R_{\tilde{s}_{0}}(2 \Delta) & R_{\tilde{s}_{0}}(\Delta) & \ldots & R_{\tilde{s}_{0}}^{*}((M-3) \Delta) \\
\vdots & \vdots & \ddots & \vdots \\
R_{\tilde{s}_{0}}((M-1) \Delta) & R_{\tilde{s}_{0}}((M-2) \Delta) & \ldots & R_{\tilde{s}_{0}}(0)
\end{array}\right]
$$

Assim considerando (3-58), a expressão em (3-55) é dada por

$$
\mathbf{s} \sim \mathcal{C N}\left(\mathbf{0}, \beta \mathbf{K}_{\mathbf{s}_{0}}\right)
$$

\section{5}

\section{Modelagem do vetor de observações}

A decisão sobre a disponibilidade da banda licenciada na qual o usuário secundário deseja operar é baseada nas amostras da envoltória complexa do sinal observado $r(t)$. Estas amostras formam o vetor aleatório de observações $\mathbf{r}$ dado por (3-12). Em (3-12), o vetor de ruído $\mathbf{n}$ tem sua caracterização estatística dada por (3-36). Observe que, no caso da modelagem determinística do sinal do usuário primário, o vetor de sinal s é dado por (3-40) enquanto que na modelagem probabilística do sinal do usuário primário a caracterização estatística do vetor s é dada por (3-60). Assim, no caso da modelagem determinística do sinal do usuário primário, descrita na Seção 3.4.1, tem-se

$$
\mathbf{r}=\left\{\begin{array}{lll}
H_{0}: & \sim \mathcal{C N}\left(\mathbf{0}, 2 \sigma_{n}^{2} \mathbf{I}\right) \\
H_{1}: & \sim \mathcal{C N}\left(\mathbf{s}, 2 \sigma_{n}^{2} \mathbf{I}\right)
\end{array}\right.
$$

com $\mathbf{s}$ denotando o vetor das amostras da envoltória complexa do sinal determinístico $s(t)$.

Já no caso da modelagem probabilística do sinal do usuário primário, descrita na Seção 3.4.2, tem-se 


$$
\mathbf{r}=\left\{\begin{array}{rll}
H_{0}: & \sim \mathcal{C N}\left(\mathbf{0}, 2 \sigma_{n}^{2} \mathbf{I}\right) \\
H_{1}: & \sim \mathcal{C N}\left(\mathbf{0}, \beta \mathbf{K}_{\mathbf{s}_{0}}+2 \sigma_{n}^{2} \mathbf{I}\right)
\end{array}\right.
$$

com $\mathbf{K}_{\mathbf{s}_{0}}$ dado por (3-59). Note que, na obtenção de (3-62), considerou-se que os vetores $\mathbf{s}$ e $\mathbf{n}$ são estatisticamente independentes.

\section{6}

\section{Critério de detecção de Neyman-Pearson}

Usualmente no problema de detecção de espectro no Rádio Cognitivo, definido como um problema de hipóteses binário, as probabilidades da presença ou ausência do usuário primário (probabilidades a priori das hipóteses $H_{0}$ e $H_{1}$ ) são desconhecidas. Assim, para resolver o problema do teste de hipóteses binário procura-se utilizar um critério de decisão que não necessite do conhecimento destas probabilidades a priori.

Um procedimento para contornar essa dificuldade é trabalhar com probabilidades condicionais como as probabilidades de detecção $P_{d}$ e de falso alarme $P_{f a}$, como o faz o critério de Neyman-Pearson que, para um dado valor de $P_{f a}$, maximiza a probabilidade de detecção $P_{d}$. A solução deste problema de otimização resulta num teste de razão de verossimilhança [?] onde o limiar de decisão é calculado a partir do valor especificado para a probabilidade de falso alarme. Matematicamente, a decisão é feita a partir do teste

$$
\Lambda(\mathbf{R})=\frac{p_{\mathbf{r} \mid H_{1}}(\mathbf{R})}{p_{\mathbf{r} \mid H_{0}}(\mathbf{R})} \underset{H_{0}}{\gtrless} \mu
$$

onde o limiar de decisão $\lambda$ é determinado a partir da condição

$$
P_{f a}=P\left(\Lambda(\mathbf{r}) \geqslant \mu \mid H_{0}\right)=\alpha \leq \alpha^{\prime}
$$

sendo $\alpha^{\prime}$ o valor especificado para a probabilidade de falso alarme.

Considerando-se que o vetor de observações $\mathbf{r}$ é, em cada uma das hipóteses, caracterizado por um vetor aleatório complexo gaussiano de média nula, o teste de razão de verossimilhança em (3-63) se escreve

$$
\Lambda(\mathbf{R})=\frac{\frac{1}{\pi^{M} \operatorname{det}\left(\mathbf{K}_{\mathbf{r} \mid H_{1}}\right)} \exp -\mathbf{R}^{\mathcal{H}} \mathbf{K}_{\mathbf{r} \mid H_{1}}^{-1} \mathbf{R}}{\frac{1}{\pi^{M} \operatorname{det}\left(\mathbf{K}_{\mathbf{r} \mid H_{0}}\right)} \exp -\mathbf{R}^{\mathcal{H}} \mathbf{K}_{\mathbf{r} \mid H_{0}}^{-1} \mathbf{R}} \underset{H_{0}}{\stackrel{H_{1}}{⿲}} \mu
$$

onde $\mathbf{K}_{\mathbf{r} \mid H_{0}}$ e $\mathbf{K}_{\mathbf{r} \mid H_{1}}$ denotam, respectivamente, as matrizes covariância do vetor r nas hipóteses $H_{0}$ e $H_{1}$.

Após algumas manipulações matemáticas o teste de razão de verossimilhança em (3-63) torna-se

com

$$
\mathbf{R}^{\mathcal{H}} \mathbf{A R} \underset{H_{0}}{\gtrless} \eta^{\prime}
$$




$$
\mathbf{A}=\mathbf{K}_{\mathbf{r} \mid H_{0}}^{-1}-\mathbf{K}_{\mathbf{r} \mid H_{1}}^{-1}
$$

$\mathrm{e}$

$$
\eta^{\prime}=\ln (\mu)-\ln \left(\frac{\operatorname{det}\left(\mathbf{K}_{\mathbf{r} \mid H_{0}}\right)}{\operatorname{det}\left(\mathbf{K}_{\mathbf{r} \mid H_{1}}\right)}\right)
$$

No caso particular em que o sinal do usuário primário é modelado por um processo estocástico gaussiano ESA de média nula, conforme descrito na Seção 3.4.2, a caracterização do vetor $\mathbf{r}$ em cada uma das hipóteses é dada por (3-62). Neste caso, a matriz A em (3-67) e o limiar $\eta^{\prime}$ em (3-68) são dados, respectivamente, por

$$
\begin{gathered}
\mathbf{A}=\mathbf{K}_{\mathbf{n}}^{-1}-\left(\beta \mathbf{K}_{\mathbf{s}_{0}}+\mathbf{K}_{\mathbf{n}}\right)^{-1} \\
\eta^{\prime}=\ln (\mu)-\ln \left(\frac{\operatorname{det}\left(\mathbf{K}_{\mathbf{n}}\right)}{\operatorname{det}\left(\beta \mathbf{K}_{\mathbf{s}_{0}}+\mathbf{K}_{\mathbf{n}}\right)}\right) .
\end{gathered}
$$

Uma expressão alternativa para a matriz A pode ser obtida considerandose a matriz $\mathbf{P}$ cujas linhas são os autovetores ortonormais $\left\{\mathbf{e}_{1}, \mathbf{e}_{2}, \ldots \mathbf{e}_{n}\right\}$ da matriz covariância $\mathbf{K}_{\mathbf{s}_{0}}$ definida em (3-59). Estes autovetores satisfazem à condição

$$
\mathbf{K}_{\mathbf{s}_{0}} \mathbf{e}_{i}=\lambda_{i} \mathbf{e}_{i} \quad ; \quad i=1, \ldots, M
$$

onde $\left\{\lambda_{i} ; i=1, \ldots, M\right\}$ representam os autovalores de $\mathbf{K}_{\mathbf{s}_{0}}$ [43]. Note que, por serem ortonormais, os autovetores $\left\{\mathbf{e}_{1}, \mathbf{e}_{2}, \ldots \mathbf{e}_{M}\right\}$ satisfazem à condição

$$
\mathbf{e}_{i}^{\mathcal{H}} \mathbf{e}_{j}=\left\{\begin{array}{lll}
1 & : & i=j \\
0 & : & i \neq j
\end{array}\right.
$$

e a matriz $\mathbf{P}$ é unitária, ou seja, $\mathbf{P}$ é uma matriz quadrada complexa tal que

$$
\mathbf{P}^{\mathcal{H}} \mathbf{P}=\mathbf{P P}^{\mathcal{H}}=\mathbf{I}
$$

Observe que se $\mathbf{P}$ é unitária seu transposto conjugado $\mathbf{P}^{\mathcal{H}}$ é também sua inversa.

Seja s' o vetor definido pela transformação linear

$$
\mathbf{s}^{\prime}=\mathbf{P} \mathbf{s}
$$

ou ainda, considerando (3-52),

$$
\mathbf{s}^{\prime}=\sqrt{\beta} \mathbf{P} \mathbf{s}_{0}
$$

sendo a matriz covariância do vetor $\mathbf{s}^{\prime}$, dada por

$$
\mathbf{K}_{\mathbf{s}^{\prime}}=\mathbf{P} \mathbf{K}_{\mathbf{s}} \mathbf{P}^{\mathcal{H}}
$$

ou, considerando (3-58),

$$
\mathbf{K}_{\mathbf{s}^{\prime}}=\beta \mathbf{P K}_{\mathbf{s}_{0}} \mathbf{P}^{\mathcal{H}} .
$$

Mostra-se facilmente que, como as linhas da matriz $\mathbf{P}$ são autovetores orto- 
normais de $\mathbf{K}_{\mathbf{s}_{0}}$, (3-77) pode ser escrita como

$$
\mathbf{K}_{\mathbf{s}^{\prime}}=\beta\left[\begin{array}{cccc}
\lambda_{1} & 0 & \ldots & 0 \\
0 & \lambda_{2} & \ldots & 0 \\
\vdots & \vdots & \ddots & \vdots \\
0 & 0 & \ldots & \lambda_{M}
\end{array}\right]
$$

ou, em notação mais compacta,

$$
\mathbf{K}_{\mathbf{s}^{\prime}}=\beta \operatorname{diag}\left(\left\{\lambda_{i}\right\}_{1}^{M}\right)
$$

onde $\operatorname{diag}\left(\left\{\lambda_{i}\right\}_{1}^{M}\right)$ denota a matriz diagonal cuja diagonal contém os elementos $\lambda_{1}, \lambda_{2}, \ldots, \lambda_{M}$.

Note que, a partir de (3-77), tem-se

$$
\mathbf{P}^{\mathcal{H}} \mathbf{K}_{\mathbf{s}^{\prime}} \mathbf{P}=\beta \mathbf{P}^{\mathcal{H}} \mathbf{P} \mathbf{K}_{\mathbf{s}_{0}} \mathbf{P}^{\mathcal{H}} \mathbf{P}
$$

que, considerando-se (3-73), se escreve

$$
\mathbf{P}^{\mathcal{H}} \mathbf{K}_{\mathbf{s}^{\prime}} \mathbf{P}=\beta \mathbf{K}_{\mathbf{s}_{0}} .
$$

O relacionamento em (3-81) permite escrever a matriz A, definida em (3-69), como

$$
\mathbf{A}=\mathbf{K}_{\mathbf{n}}^{-1}-\left(\mathbf{P}^{\mathcal{H}} \mathbf{K}_{\mathbf{s}^{\prime}} \mathbf{P}+\mathbf{K}_{\mathbf{n}}\right)^{-1}
$$

A partir do Lema de inversão de matrizes [44], dado por

$$
(\mathbf{E}+\mathbf{F G H})^{-1}=\mathbf{E}^{-1}-\mathbf{E}^{-1} \mathbf{F}\left(\mathbf{H E}^{-1} \mathbf{F}+\mathbf{G}^{-1}\right)^{-1} \mathbf{H} \mathbf{E}^{-1},
$$

é possível escrever a matriz A em (3-82) na forma

$$
\mathbf{A}=\mathbf{K}_{\mathbf{n}}^{-1} \mathbf{P}^{\mathcal{H}} \mathbf{D P} K_{\mathbf{n}}^{-1}
$$

com

$$
\mathbf{D}=\left(\mathbf{P} \mathbf{K}_{\mathbf{n}}^{-1} \mathbf{P}^{\mathcal{H}}+\mathbf{K}_{\mathbf{s}^{\prime}}^{-1}\right)^{-1}
$$

ou, levando-se em conta (3-79),

$$
\mathbf{D}=\left(\mathbf{P} \mathbf{K}_{\mathbf{n}}^{-1} \mathbf{P}^{\mathcal{H}}+\operatorname{diag}\left(\left\{\frac{1}{\beta \lambda_{i}}\right\}_{1}^{M}\right)\right)^{-1} .
$$

Considerando-se o caso particular de ruído branco, ou seja $\mathbf{K}_{\mathbf{n}}=2 \sigma_{n}^{2} \mathbf{I}$, tem-se

$$
\mathbf{P K}_{\mathbf{n}}^{-1} \mathbf{P}^{\mathcal{H}}=\frac{1}{2 \sigma_{n}^{2}} \mathbf{P} \mathbf{P}^{\mathcal{H}}=\frac{1}{2 \sigma_{n}^{2}} \mathbf{I}
$$

e, consequentemente, (3-86) torna-se

$$
\mathbf{D}=\left(\frac{1}{2 \sigma_{n}^{2}} \mathbf{I}+\operatorname{diag}\left(\left\{\frac{1}{\beta \lambda_{i}}\right\}_{1}^{M}\right)\right)^{-1}
$$

ou ainda, 


$$
\mathbf{D}=\operatorname{diag}\left(\left\{\frac{2 \sigma_{n}^{2} \beta \lambda_{i}}{\beta \lambda_{i}+2 \sigma_{n}^{2}}\right\}_{1}^{M}\right)
$$

Em notação mais compacta,

$$
\mathbf{D}=\operatorname{diag}\left(\left\{a_{i}\right\}_{1}^{M}\right)
$$

com

$$
a_{i}=\frac{2 \sigma_{n}^{2} \beta \lambda_{i}}{\beta \lambda_{i}+2 \sigma_{n}^{2}} \quad ; \quad i=1, \ldots, M
$$

ou ainda,

$$
a_{i}=\frac{\beta \lambda_{i}}{\frac{\beta \lambda_{i}}{2 \sigma_{n}^{2}}+1} \quad ; \quad i=1, \ldots, M .
$$

Note que os elementos $\left\{a_{i}, i=1, \ldots, M\right\}$ da matriz diagonal $\mathbf{D}$ dependem da potência média $\beta$ da envoltória complexa do sinal do usuário primário.

Considerando-se a definição da matriz A em (3-84) e a definição da matriz D em (3-90), o teste em (3-66) pode ser reescrito como

$$
v(\mathbf{R})=\mathbf{R}^{\mathcal{H}} \mathbf{P}^{\mathcal{H}} \operatorname{diag}\left(\left\{a_{i}\right\}_{1}^{M}\right) \mathbf{P R} \underset{H_{0}}{\stackrel{H_{1}}{\gtrless}} \eta
$$

com $v(\mathbf{R})$ caracterizando a variável de decisão, e $\eta$ o limiar de decisão dado por

$$
\eta=\left(2 \sigma_{n}^{2}\right)^{2}\left(\ln (\mu)-\ln \left(\frac{\operatorname{det}\left(\mathbf{K}_{\mathbf{n}}\right)}{\operatorname{det}\left(\beta \mathbf{K}_{\mathbf{s}_{0}}+\mathbf{K}_{\mathbf{n}}\right)}\right)\right)
$$

Note que, de acordo com o critério de Neyman-Pearson, o limiar $\eta$ é determinado, conforme indicado em (3-64), fixando-se um valor máximo para a probabilidade de falso alarme, ou seja, resolvendo-se a equação

$$
P_{f a}=P\left(v(\mathbf{r}) \geqslant \eta \mid H_{0}\right)=\alpha \leq \alpha^{\prime}
$$

\section{7}

\section{Métricas de desempenho do detector}

O desempenho do detector em (3-93) é usualmente avaliado por duas métricas amplamente conhecidas: a probabilidade de falso alarme $P_{f a}$, que avalia a probabilidade de decidir pela presença de sinal de usuário primário na faixa de frequência observada quando na realidade não existe transmissões do usuário primário, e a probabilidade de detecção $P_{d}$, que fornece a probabilidade de detectar corretamente a presença de sinal do usuário primário na faixa de frequência observada. Matematicamente $P_{f a}$ e $P_{d}$ são definidas, respectivamente, por [?]

$$
P_{f a}=P\left(v>\eta \mid H_{0}\right)=\int_{\eta}^{\infty} p_{v \mid H_{0}}(V) d V
$$

$\mathrm{e}$

$$
P_{d}=P\left(v>\eta \mid H_{1}\right)=\int_{\eta}^{\infty} p_{v \mid H_{1}}(V) d V
$$


Note que as definições matemáticas das probabilidades $P_{f a}$ e $P_{d}$ dependem das funções densidade de probabilidade condicionais da variável de decisão $v$, dadas as hipóteses $H_{0}$ e $H_{1}$, respectivamente.

Visando a determinação de expressões matemáticas para $p_{v \mid H_{0}}(V)$ e $p_{v \mid H_{1}}(V)$, considere o vetor $\mathbf{x}$ definido por

$$
\mathbf{x}=\mathbf{P r}
$$

onde $\mathbf{P}$ é a matriz de autovetores de $\mathbf{K}_{\mathbf{s}_{0}}$ definida em (3-71) e (3-72) e $\mathbf{r}$ é o vetor aleatório complexo observado caraterizado em (3-62). Considerando-se que o vetor complexo $\mathbf{r}$ é gaussiano e circularmente simétrico (ver (3-62)), o relacionamento linear em (3-98) permite concluir que $\mathbf{x}$ é também um vetor gaussiano complexo, circularmente simétrico [36], ou seja,

$$
\mathbf{x} \sim \mathcal{C N}\left(\mathbf{m}_{\mathbf{x}}, \mathbf{K}_{\mathbf{x}}\right)
$$

onde $\mathbf{m}_{\mathbf{x}}$ e $\mathbf{K}_{\mathbf{x}}$ representam respectivamente o vetor média e a matriz covariância do vetor $\mathbf{x}$. Note que

$$
\mathbf{m}_{\mathbf{x}}=\mathbf{P m}_{\mathbf{r}}
$$

e

$$
\mathbf{K}_{\mathbf{x}}=\mathbf{P} \mathbf{K}_{\mathbf{r}} \mathbf{P}^{\mathcal{H}}
$$

Considerando-se (3-98), a variável de decisão $v$ definida em (3-93) se escreve

$$
v=\mathbf{x}^{\mathcal{H}} \operatorname{diag}\left(\left\{a_{i}\right\}_{1}^{M}\right) \mathbf{x}
$$

ou ainda,

$$
v=\sum_{i=1}^{M} y_{i}
$$

com

$$
y_{i}=a_{i}\left|x_{i}\right|^{2}, \quad ; \quad i=1, \ldots, M
$$

Em (3-104), as variáveis $\left\{x_{i}, i=1, \ldots, M\right\}$ correspondem às componentes do vetor $\mathbf{x}$, aqui caracterizadas como variáveis aleatórias complexas, circularmente simétricas e conjuntamente gaussianas. Note que cada uma destas variáveis aleatórias é gaussiana, complexa e circularmente simétrica, ou seja,

$$
x_{i} \sim \mathcal{C N}\left(m_{x_{i}}, 2 \sigma_{x_{i}}^{2}\right) \quad ; \quad i=1,2, \ldots, M
$$

onde $m_{x_{i}}$ e $2 \sigma_{x_{i}}^{2}$ denotam respectivamente a média e a variância da variável aleatória $x_{i}$. Este fato implica (se $m_{x_{i}}=0$ ) em que as variáveis aleatórias $\left\{\left|x_{i}\right|^{2}, i=1, \ldots, M\right\}$ têm, cada uma delas, função densidade de probabilidade exponencial de parâmetro $1 / 2 \sigma_{x_{i}}^{2}$ [37]. Neste caso, considerando-se (3-104), mostra-se facilmente que cada uma das parcelas do somatório em (3-103) é uma variável aleatória com função densidade de probabilidade exponencial, dada por

$$
p_{y_{i}}(Y)=c_{i} e^{-c_{i} Y} u(Y) \quad ; \quad c_{i}>0
$$


com o parâmetro $c_{i}$ dado por

$$
c_{i}=\frac{1}{a_{i} 2 \sigma_{x_{i}}^{2}}
$$

onde $a_{i}$ é dado por (3-91). Em (3-106), u() representa a função degrau unitário, definida por

$$
u(X)= \begin{cases}0 & X<0 \\ 1 & X \geq 0\end{cases}
$$

Em resumo, a variável de decisão $v$ em (3-93) ou (3-102) corresponde à soma de variáveis aleatórias com função densidade de probabilidade exponencial.

\subsection{1}

\section{Funções densidade de probabilidade condicionais da variável de decisão}

Nesta seção, considera-se o caso mais geral descrito na Seção 3.4.2, onde o sinal interferente produzido pelas transmissões de usuários primários é caracterizado por um processo estocástico gaussiano ESA, de média nula. Neste caso, a caracterização do vetor de observações $\mathbf{r}$ é dada por (3-62), indicando que, se a hipótese $H_{0}$ é verdadeira, ou seja, na ausência de sinal interferente devido ao UP, o vetor complexo $\mathbf{r}$ tem média nula e matriz covariância $\mathbf{K}_{\mathbf{r} \mid H_{0}}=2 \sigma_{n}^{2} \mathbf{I}$. Assim, considerando-se (3-100) e (3-101), obtémse, respectivamente,

$$
\mathbf{m}_{\mathbf{x}_{\mid H_{0}}}=\mathbf{P m}_{\mathbf{r} \mid H_{0}}=\mathbf{0}
$$

$\mathrm{e}$

$$
\mathbf{K}_{\mathbf{x}_{\mid H_{0}}}=\mathbf{P} \mathbf{K}_{\mathbf{r} \mid H_{0}} \mathbf{P}^{\mathcal{H}}=2 \sigma_{n}^{2} \mathbf{P} \mathbf{P}^{\mathcal{H}}
$$

ou ainda, considerando-se (3-73),

$$
\mathbf{K}_{\mathbf{x}_{\mid H_{0}}}=2 \sigma_{n}^{2} \mathbf{I}
$$

Isto significa que, dado $H_{0}$, as componentes do vetor complexo $\mathbf{x}$ em (3-98), caracterizadas pelas variáveis aleatórias complexas $\left\{x_{i}, i=1, \ldots, M\right\}$, são descorrelatadas duas a duas e, por serem conjuntamente gaussianas, são também estatisticamente independentes. Consequentemente, dado $H_{0}$, as variáveis aleatórias $\left\{y_{i}, i=1, \ldots, M\right\}$ definidas em (3-104), além de terem funções densidade de probabilidade exponenciais, ou seja,

$$
p_{y_{i} \mid H_{0}}(Y)=c_{i} e^{-c_{i} Y} u(Y) \quad ; \quad c_{i}>0
$$

com

$$
c_{i}=\frac{1}{a_{i} 2 \sigma_{n}^{2}},
$$

são estatisticamente independentes. Em (3-113), o coeficiente $a_{i}$ é dado por (3-91).

Em resumo, dado $H_{0}$, a variável de decisão $v$ em (3-103), corresponde à 
soma de variáveis aleatórias estatisticamente independentes, cada uma delas com função densidade de probabilidade exponencial. No Apêndice A, são apresentados dois procedimentos para a determinação da função densidade de probabilidade desta soma. A implementação do primeiro deles, procedimento mais conhecido, apresentou problemas de ordem numérica quando número de parcelas é muito grande (e.g. $M>400$ ) e quando os parâmetros $c_{i}$ de cada uma das parcelas são muito próximos, o que ocorre no caso em análise. Assim, este trabalho utiliza o segundo procedimento apresentado no Apêndice A (Seção A.2), baseado na série convergente proposta em [1]. De acordo com este procedimento, a função densidade de probabilidade da variável aleatória $v$ dado $H_{0}$ pode ser expressa por

$$
p_{v \mid H_{0}}(V)=C \sum_{k=0}^{\infty} \delta_{k} \frac{V^{M+k-1}\left(c^{*}\right)^{M+k}}{\Gamma(M+k)} \exp \left(-c^{*} V\right) u(V)
$$

onde, considerando-se (A-12), (3-91) e (3-113),

$$
c^{*}=\frac{1}{\left(2 \sigma_{n}^{2}\right)^{2}}\left(1+\frac{2 \sigma_{n}^{2}}{\beta \lambda^{*}}\right)
$$

com

$$
\lambda^{*}=\min _{i} \lambda_{i}
$$

$\mathrm{e}$

$$
C=\left(1+\frac{2 \sigma_{n}^{2}}{\beta \lambda^{*}}\right)^{-M} \prod_{i=1}^{M}\left(1+\frac{2 \sigma_{n}^{2}}{\beta \lambda_{i}}\right) .
$$

Conforme indicado em (A-14) e (A-15), considerando-se (3-91) e (3-113), os coeficientes $\delta_{k}$ em (3-114), são dados por

$$
\delta_{k}= \begin{cases}1 & ; k=0 \\ \frac{1}{k} \sum_{i=1}^{k} i \gamma_{i} \delta_{k-i} & ; k=1,2, \ldots\end{cases}
$$

onde

$$
\gamma_{k}=\frac{1}{k}\left(\frac{2 \sigma_{n}^{2} \lambda^{*}}{\beta \lambda^{*}+2 \sigma_{n}^{2}}\right)^{k} \sum_{\ell=1}^{M}\left(\frac{1}{\lambda^{*}}-\frac{1}{\lambda_{\ell}}\right)^{k} ; \quad k=1,2,3 \ldots
$$

Caso a hipótese $H_{1}$ seja verdadeira, ou seja, existe interferência produzida pelas transmissões de usuários primários, a caracterização do vetor de observações $\mathbf{r}$ em (3-62), indica que o vetor complexo $\mathbf{r}$ tem média nula e matriz covariância $\mathbf{K}_{\mathbf{r} \mid H_{1}}=\beta \mathbf{K}_{\mathbf{s}_{0}}+2 \sigma_{n}^{2} \mathbf{I}$. Neste caso, considerando-se (3-100) e (3-101), obtém-se, respectivamente,

$$
\mathbf{m}_{\mathbf{x}_{\mid H_{1}}}=\mathbf{P m}_{\mathbf{r} \mid H_{1}}=\mathbf{0}
$$

$\mathrm{e}$

$$
\mathbf{K}_{\mathbf{x}_{\mid H_{1}}}=\mathbf{P K}_{\mathbf{r} \mid H_{1}} \mathbf{P}^{\mathcal{H}}=\beta \mathbf{P K}_{\mathbf{s}_{0}} \mathbf{P}^{\mathcal{H}}+2 \sigma_{n}^{2} \mathbf{P} \mathbf{P}^{\mathcal{H}}
$$

ou ainda, considerando (3-71), (3-72), (3-73), (3-77) e (3-78), 


$$
\mathbf{K}_{\mathbf{x}_{\mid H_{1}}}=\left[\begin{array}{cccc}
\beta \lambda_{1}+2 \sigma_{n}^{2} & 0 & \ldots & 0 \\
0 & \beta \lambda_{2}+2 \sigma_{n}^{2} & \ldots & 0 \\
\vdots & \vdots & \ddots & \vdots \\
0 & 0 & \ldots & \beta \lambda_{M}+\sigma_{n}^{2}
\end{array}\right]
$$

Em notação mais compacta,

$$
\mathbf{K}_{\mathbf{x}_{\mid H_{1}}}=\operatorname{diag}\left(\left\{\beta \lambda_{i}+2 \sigma_{n}^{2}\right\}_{1}^{M}\right)
$$

A partir de (3-122) (ou (3-123)) é possível identificar que as componentes do vetor complexo $\mathbf{x}$ em (3-98), ou seja, as variáveis aleatórias complexas $\left\{x_{i}, i=1, \ldots, M\right\}$ são descorrelatadas duas a duas e, por serem conjuntamente gaussianas são estatisticamente independentes. Consequentemente, dado $H_{1}$, as variáveis aleatórias $\left\{y_{i}, i=1, \ldots, M\right\}$ definidas em (3-104), além de terem funções densidade de probabilidade exponenciais, ou seja,

$$
p_{y_{i} \mid H_{1}}(Y)=c_{i} e^{-c_{i} Y} u(Y) \quad ; \quad c_{i}>0
$$

com

$$
c_{i}=\frac{1}{a_{i}\left(\beta \lambda_{i}+2 \sigma_{n}^{2}\right)}
$$

são estatisticamente independentes. Em (3-125), o coeficiente $a_{i}$ é dado por (3-91).

Em resumo, dado $H_{1}$, a variável de decisão $v$ em (3-103), também corresponde à soma de variáveis aleatórias estatisticamente independentes, cada uma delas com função densidade de probabilidade exponencial. Novamente, o segundo procedimento apresentado no Apêndice A para a determinação da função densidade de probabilidade da soma de variáveis aleatórias com distribuição exponencial e estatisticamente independentes é utilizado, resultando

$$
p_{v \mid H_{1}}(V)=C \sum_{k=0}^{\infty} \delta_{k} \frac{V^{M+k-1}\left(c^{*}\right)^{M+k}}{\Gamma(M+k)} \exp \left(-c^{*} V\right) u(V)
$$

onde, considerando-se (A-12), (3-91) e (3-125),

$$
c^{*}=\frac{1}{2 \sigma_{n}^{2} \beta \lambda^{*}}
$$

com

$$
\lambda^{*}=\min _{i} \lambda_{i}
$$

e

$$
C=\prod_{i=1}^{M} \frac{\lambda^{*}}{\lambda_{i}}
$$

Conforme indicado em (A-14) e (A-15), considerando-se (3-91) e (3-125), os coeficientes $\delta_{k}$ em (3-126), são dados por 


$$
\delta_{k}= \begin{cases}1 & ; k=0 \\ \frac{1}{k} \sum_{i=1}^{k} i \gamma_{i} \delta_{k-i} & ; k=1,2, \ldots\end{cases}
$$

onde

$$
\gamma_{k}=\sum_{\ell=1}^{M} \frac{\left(1-\frac{\lambda^{*}}{\lambda_{\ell}}\right)^{k}}{k} ; \quad k=1,2,3 \ldots
$$

onde $\left\{\lambda_{i} ; i=1, \ldots, M\right\}$ representam os autovalores de $\mathbf{K}_{\mathbf{s}_{0}}$.

\subsection{2}

\section{Probabilidade de falso alarme e probabilidade de detecção}

A partir da definição da probabilidade de falso alarme, dada por (3-96), e do conhecimento da função densidade de probabilidade condicional da variável de decisão $v$ dada a hipótese $H_{0}$ (ver (3-114)), a probabilidade de falso alarme se escreve

$$
P_{f a}=C \sum_{k=0}^{\infty} \frac{\delta_{k}}{\Gamma(M+k)}\left(c^{*}\right)^{M+k} \int_{\eta}^{\infty} V^{M+k-1} \exp \left(-c^{*} V\right) d V
$$

ou ainda, fazendo-se a mudança de variável de integração $z=c^{*} V$,

$$
P_{f a}=C \sum_{k=0}^{\infty} \frac{\delta_{k}}{\Gamma(M+k)}\left(c^{*}\right)^{M+k} \int_{c^{*} \eta}^{\infty}\left(\frac{Z}{c^{*}}\right)^{M+k-1} \frac{1}{c^{*}} \exp (-Z) d Z .
$$

Após algumas manipulações matemáticas esta probabilidade pode ainda ser escrita como

$$
P_{f a}=C \sum_{k=0}^{\infty} \delta_{k} \frac{\Gamma\left(M+k, c^{*} \eta\right)}{\Gamma(M+k)}
$$

onde $\Gamma(\cdot, \cdot)$ denota a Função Gama Incompleta Superior [45] definida por

$$
\Gamma(n, \alpha)=\int_{\alpha}^{\infty} Z^{n-1} \exp (-Z) d Z \quad ; \quad \mathbb{R}(n)>0
$$

e $\Gamma(\cdot)$ representa a Função Gama [35] definida por

$$
\Gamma(n)=\int_{0}^{\infty} Z^{n-1} \exp (-Z) d Z \quad ; \quad \mathbb{R}(n)>0
$$

em (3-134), $c^{*}, C$ e $\delta_{k}$ são dados por (3-115), (3-117) e (3-118), respectivamente.

A partir da definição da probabilidade de detecção, dada por (3-97), e do conhecimento da função densidade de probabilidade condicional da variável de decisão $v$ dada a hipótese $H_{1}$ (ver (3-126)), a probabilidade de detecção se escreve

$$
P_{d}=C \sum_{k=0}^{\infty} \frac{\delta_{k}}{\Gamma(M+k)}\left(c^{*}\right)^{M+k} \int_{\eta}^{\infty} V^{M+k-1} \exp \left(-c^{*} V\right) d V
$$

Fazendo-se a mudança de variável de integração $z=c^{*} V$ obtém-se, após algumas manipulações matemáticas, 


$$
P_{d}=C \sum_{k=0}^{\infty} \delta_{k} \frac{\Gamma\left(M+k, c^{*} \eta\right)}{\Gamma(M+k)}
$$

onde $\Gamma(\cdot, \cdot)$ denota a Função Gama Incompleta Superior [45] definida em (3-135) e $\Gamma(\cdot)$ representa a Função Gama [35] definida em (3-136). Em (3-138), $c^{*}, C$ e $\delta_{k}$ são dados por (3-127), (3-129) e (3-130), respectivamente.

Note que o limiar de decisão $\eta$ a ser utilizado no teste de hipóteses em (3-93) é obtido utilizando-se a condição em (3-95). Assim, considerando-se (3-134), este limiar de decisão é obtido resolvendo-se a equação

$$
C \sum_{k=0}^{\infty} \delta_{k} \frac{\Gamma\left(M+k, c^{*} \eta\right)}{\Gamma(M+k)}=\alpha^{\prime}
$$

É importante observar que a expressão no lado esquerdo de (3-139), além de depender de $\eta$, depende também de $\beta$. Assim, especificado o valor $\alpha^{\prime}$ a ser utilizado, é possível resolver (3-139) para diferentes valores de $\beta$, obtendo-se uma curva de $\eta$ versus $\beta$. A Figura 3.5 mostra o exemplo de uma curva deste tipo, obtida para $\alpha^{\prime}=10^{-3}$. Neste exemplo foram utilizados os parâmetros técnicos descritos nas seções 5.1 e 5.2 para os sistemas FS e HDFSS.

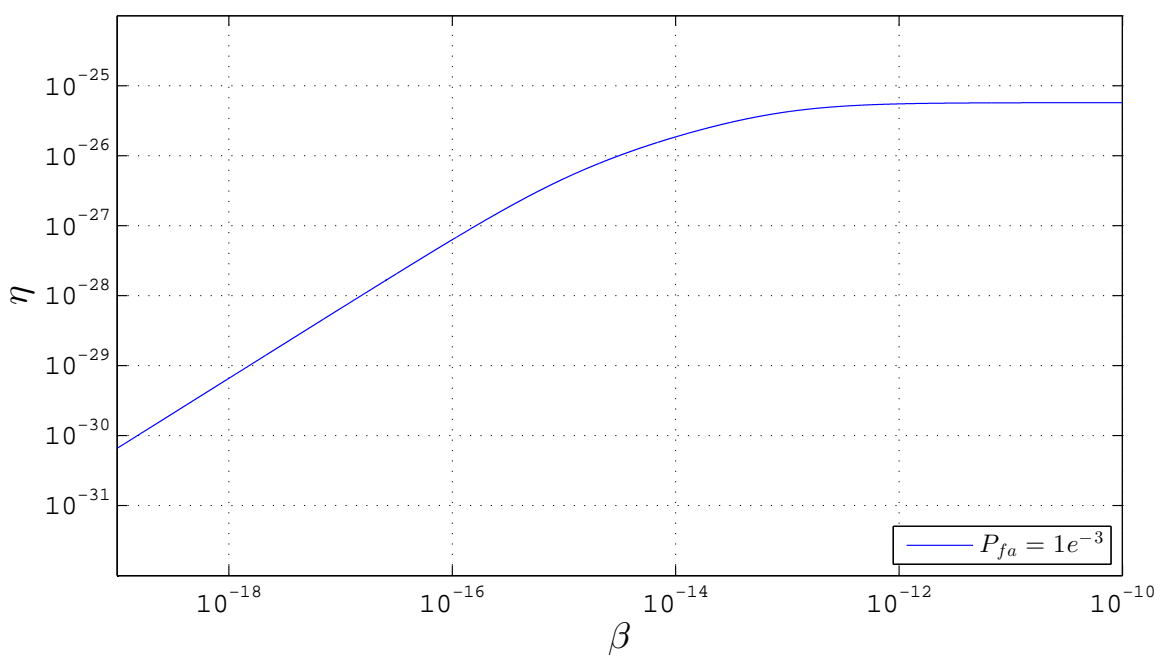

Figura 3.5: Valores do limiar a ser utilizado no teste de hipóteses em função da potência média $\beta$.

\section{8}

\section{Estimador da potência média da envoltória complexa do sinal do usuário primário}

Duas possibilidades são consideradas para a estimação da potência média $\beta$ da envoltória complexa do sinal do usuário primário: estimativa feita a partir da observação das amostras da envoltória complexa do sinal recebido (elementos do vetor $\mathbf{r}$ ) e estimativa feita a partir do valor observado da variável de decisão $v$. Estas duas possibilidades são abordadas nas subseções seguintes. 


\subsection{1}

\section{Estimação a partir do vetor de observações $r$}

Com base no vetor aleatório de observações $\mathbf{r}$, obtido a partir de $M$ amostras da envoltória complexa do sinal observado $r(t)$, cada usuário secundário deve calcular e enviar ao DBC o valor assumido pela variável de decisão $v$. Note que, esta variável dada por (3-102), depende da potência média $\beta$ da envoltória complexa do sinal do usuário primário cujo valor é desconhecido e precisa ser estimado pelo US a partir do vetor observado r. Uma possibilidade é a utilização do estimador de máxima verossimilhança (ML - Maximum Likelihood) que é definido por [?]

$$
\hat{\beta}_{m l}(\mathbf{R})=\max _{B}^{-1}\left[P\left(H_{0}\right) p_{\mathbf{r} \mid \beta=B, H_{0}}(\mathbf{R})+P\left(H_{1}\right) p_{\mathbf{r} \mid \beta=B, H_{1}}(\mathbf{R})\right]
$$

A partir da caraterização do vetor de observações em (3-62), observa-se que a função densidade de probabilidade $p_{\mathbf{r} \mid \beta=B, H_{0}}(\mathbf{R})$ não depende do parâmetro $\beta$, $\operatorname{assim}(3-140)$ se reduz

$$
\hat{\beta}_{m l}(\mathbf{R})=\max _{B}^{-1}\left[p_{\mathbf{r} \mid \beta=B, H_{1}}(\mathbf{R})\right]
$$

ou ainda

$$
\hat{\beta}_{m l}(\mathbf{R})=\max _{B}^{-1}\left[\ln \left(p_{\mathbf{r} \mid \beta=B, H_{1}}(\mathbf{R})\right)\right]
$$

Considerando-se a caracterização de r, (3-142) se escreve

$\hat{\beta}_{m l}(\mathbf{R})=\max _{B}^{-1}\left[-M \ln \pi-\ln \operatorname{det}\left(B \mathbf{K}_{\mathbf{s}_{0}}+2 \sigma_{n}^{2} \mathbf{I}\right)-\mathbf{R}^{\mathrm{H}}\left(B \mathbf{K}_{\mathbf{s}_{0}}+2 \sigma_{n}^{2} \mathbf{I}\right)^{-1} \mathbf{R}\right]$,

A Equação ML, a ser resolvida para a obtenção de $\hat{\beta}_{m l}(V)$ é então obtida considerando-se a condição dada por

$$
-\frac{\partial}{\partial B} \ln \operatorname{det}\left(B \mathbf{K}_{\mathbf{s}_{0}}+2 \sigma_{n}^{2} \mathbf{I}\right)-\mathbf{R}^{\mathrm{H}} \frac{\partial}{\partial B}\left(B \mathbf{K}_{\mathbf{s}_{0}}+2 \sigma_{n}^{2} \mathbf{I}\right)^{-1} \mathbf{R}=0
$$

A obtenção da solução de (3-144), que caracteriza o estimador $\hat{\beta}_{m l}$ de máxima verossimilhança do parâmetro $\beta$, é complexa e usualmente obtida por métodos numéricos. Note entretanto que, no caso particular em que a matriz $\mathbf{K}_{\mathbf{s}_{0}}$ é uma matriz diagonal, é possível se chegar a uma solução fechada para $\hat{\beta}_{m l}$, conforme será evidenciado na Subseção 3.9.4.1.

\subsection{2}

\section{Estimação a partir da observação da variável de decisão $v$}

Note que, as funções densidade de probabilidade condicionais, da variável de decisão $v, p_{v \mid H_{0}}(V)$ em (3-114) a (3-119) e $p_{v \mid H_{1}}(V)$ em (3-126) a (3-131), dependem da potência média $\beta$ da envoltória complexa do sinal do usuário primário. Esta dependência se dá através dos parâmetros $C, c^{*}$ e $\delta_{k}$ no caso de 
dada a hipótese $H_{0}$ e através do parâmetro $c^{*}$ no caso de dada a hipótese $H_{1}$. Consequentemente, a probabilidade de falso alarme dada por, (3-134), (3-115), (3-117) e (3-118), e a probabilidade de detecção, dada por (3-138), (3-127), (3-129) e (3-130), também dependem de $\beta$, cujo valor exato é desconhecido e precisa ser estimado pelo DBC com base no valor assumido pela variável de decisão $v$ definida em (3-102). Uma possibilidade é a utilização do estimador de máxima verossimilhança (ML- Maximum Likelihood), dado por [?]

$$
\hat{\beta}_{m l}(V)=\max _{B}^{-1}\left[P\left(H_{0}\right) p_{v \mid \beta=B, H_{0}}(V)+P\left(H_{1}\right) p_{v \mid \beta=B, H_{1}}(V)\right]
$$

Como as probabilidades a priori $P\left(H_{0}\right)$ e $P\left(H_{1}\right)$ em (3-145) são desconhecidas, e considerando que o sinal observado corresponde à interferência produzida pelas transmissões de usuários primários na presença de ruído térmico, propõese a utilização da estimativa subótima dada por

$$
\hat{\beta}_{1}(V)=\max _{B}^{-1}\left[p_{v \mid \beta=B, H_{1}}(V)\right]
$$

Em (3-146), a expressão da função densidade de probabilidade condicional $p_{v \mid \beta=B, H_{1}}(V)$ é dada por $(3-126), \operatorname{com} \beta=B$, ou seja,

$$
p_{v \mid \beta=B}(V)=C \sum_{k=0}^{\infty} \delta_{k} \frac{V^{M+k-1} \exp \left(-\frac{V}{2 \sigma_{n}^{2} B \lambda^{*}}\right)}{\Gamma(M+k)\left(2 \sigma_{n}^{2} B \lambda^{*}\right)^{M+k}} u(V)
$$

Assim, o estimador subótimo proposto é dado por

$$
\hat{\beta}_{1}=\max _{B}^{-1}\left[C \sum_{k=0}^{\infty} \delta_{k} \frac{V^{M+k-1} \exp \left(-\frac{V}{2 \sigma_{n}^{2} B \lambda^{*}}\right)}{\Gamma(M+k)\left(2 \sigma_{n}^{2} B \lambda^{*}\right)^{M+k}} u(V)\right]
$$

A maximização em (3-148), de difícil solução, é abordada na Seção B.1 do Apêndice B.

\section{9}

\section{Caso particular: PE gaussiano ESA com DEP plana na faixa}

Nesta Seção considera-se o caso particular amplamente abordado nos trabalhos disponíveis na literatura $[11,18,31,33,34,40-42]$, no qual o sinal produzido pelas transmissões dos usuários primários $s(t)$, é caracterizado por um processo estocástico passa-faixa gaussiano, ESA e de média nula, com densidade espectral de potência plana na faixa, ou seja,

$$
S_{s}(f)=\frac{\beta}{4 W}\left[\operatorname{ret}_{W}\left(f-f_{0}\right)+\operatorname{ret}_{W}\left(f+f_{0}\right)\right]
$$

$\operatorname{com~ret}_{W}(f)$ definido por (3-18). Neste caso, a densidade espectral de potência da envoltória complexa de $s(t)$, dada por (3-43), se escreve

$$
S_{\tilde{s}}(f)=\frac{\beta}{W} \operatorname{ret}_{W}(f)
$$


ou, em função da densidade espectral de potência da envoltória complexa normalizada $\tilde{s}_{0}(t)(\operatorname{ver}(3-47))$,

$$
S_{\tilde{s}}(f)=\beta S_{\tilde{s}_{0}}(f)
$$

com

$$
S_{\tilde{s}_{0}}(f)=\frac{1}{W} \operatorname{ret}_{W}(f)
$$

A função autocorrelação da envoltória complexa $\tilde{s}(t)$, dada por (3-45), se escreve

$$
R_{\widetilde{s}}(\tau)=\beta R_{\widetilde{s}_{0}}(\tau)
$$

onde

$$
R_{\widetilde{s}_{0}}(\tau)=\operatorname{sinc}(W \tau)
$$

com a função $\operatorname{sinc}(\cdot)$ definida em (3-23). Note que, a potência média da envoltória complexa $\tilde{s}(t)$ se escreve, neste caso

$$
P_{\tilde{s}}=R_{\tilde{s}}(0)=\beta R_{\tilde{s}_{0}}(0)=\beta
$$

Observe ainda que, de acordo com (3-154), amostras do processo estocástico complexo $\tilde{s}_{0}(t)$, tomadas a intervalos $\Delta=1 / W$ (Taxa de Nyquist), que definem o vetor complexo $\mathbf{s}_{0}$, constituem uma coleção de variáveis aleatórias complexas, conjuntamente gaussianas, descorrelatadas duas a duas (e consequentemente estatisticamente independentes), indicando que a matriz covariância de $\mathbf{s}_{0}$ é dada por

$$
\mathbf{K}_{\mathbf{s}_{0}}=\mathbf{I}
$$

com I denotando a matriz identidade. Tem-se então, a partir de (3-60), que o vetor $\mathbf{s}$ de amostras da envoltória complexa $\widetilde{\mathbf{s}}(t)$ é caracterizado por

$$
\mathbf{s} \sim \mathcal{C N}(\mathbf{0}, \beta \mathbf{I})
$$

Neste caso particular, a caracterização do vetor aleatório de observações $\mathbf{r}$, dado por (3-62), se escreve

$$
\mathbf{r}=\left\{\begin{array}{lll}
H_{0}: & \sim \mathcal{C N}\left(\mathbf{0}, 2 \sigma_{n}^{2} \mathbf{I}\right) \\
H_{1}: & \sim \mathcal{C N}\left(\mathbf{0},\left(\beta+2 \sigma_{n}^{2}\right) \mathbf{I}\right)
\end{array}\right.
$$

\subsection{1}

\section{Variável de decisão}

Note que com base na caraterização do vetor de amostras da envoltória complexa $\tilde{s}(t)$, identificou-se que a matriz covariância $\mathbf{K}_{\mathbf{s}_{0}}$ do vetor de amostras da envoltória complexa normalizada é uma matriz identidade, conforme indicado em (3-156). Assim, a matriz covariância $\mathbf{K}_{\mathbf{s}^{\prime}}$ definida em (3-77), se escreve

$$
\mathbf{K}_{\mathbf{s}^{\prime}}=\beta \mathbf{P} \mathbf{I P}^{\mathcal{H}}
$$


ou ainda, considerando-se (3-73),

$$
\mathbf{K}_{\mathbf{s}^{\prime}}=\beta \mathbf{I} .
$$

consequentemente, os elementos $\left\{\lambda_{i}, i=1, \ldots, M\right\}$ da matriz $\operatorname{diag}\left(\lambda_{i}\right)$ em (3-79) são todos iguais a 1, ou seja,

$$
\lambda_{i}=1 \quad ; \quad i=1, \ldots, M .
$$

Isto significa que os elementos $\left\{a_{i}, i=1, \ldots, M\right\}$ da matriz $\operatorname{diag}\left(a_{i}\right)$ em (3-90) são todos dados por

$$
a_{i}=\frac{2 \sigma_{n}^{2} \beta}{\beta+2 \sigma_{n}^{2}} \quad ; \quad i=1, \ldots, M
$$

Neste caso, o teste em (3-93) se torna

$$
v(\mathbf{R})=\frac{2 \sigma_{n}^{2} \beta}{\beta+2 \sigma_{n}^{2}} \mathbf{R}^{\mathcal{H}} \mathbf{R} \underset{H_{0}}{\stackrel{H_{1}}{\gtrless}} \eta
$$

com o limiar de decisão $\eta$ dado por

$$
\eta=\left(2 \sigma_{n}^{2}\right)^{2}\left(\ln (\lambda)-\ln \left(\frac{\left(2 \sigma_{n}^{2}\right)^{M}}{\left(\beta+2 \sigma_{n}^{2}\right)^{M}}\right)\right) .
$$

Note que, o teste obtido em (3-163), é equivalente ao teste

$$
\mathbf{R}^{\mathcal{H}} \mathbf{R} \underset{H_{0}}{\stackrel{H_{1}}{\gtrless}} \eta^{*}
$$

com

$$
\eta^{*}=\frac{\beta+2 \sigma_{n}^{2}}{2 \sigma_{n}^{2} \beta} \eta
$$

Convém observar que as análises em [11,18,31,33,34,40-42], que consideram que o sinal do UP tem DEP plana na faixa, utilizaram o teste de detecção na forma expressa em (3-165) e os resultados obtidos nestas análises, são compatíveis com os resultados obtidos se a forma do teste em (3-163) fosse utilizada.

\subsection{2}

\section{Funções densidade de probabilidade condicionais da variável de decisão}

A função densidade de probabilidade da variável de decisão dada a hipótese $H_{1}$, no caso da interferência $s(t)$ produzida pelas transmissões de usuários primários ser caracterizada por um PE gaussiano, ESA, de média nula e DEP genérica é dada por (3-114) a (3-119). No caso particular em que $s(t)$ tem DEP plana na faixa de interesse, $\operatorname{com}\left\{\lambda_{i}=1, i=1, \ldots, M\right\}$ (ver (3-161)), a expressão da função densidade de probabilidade condicional (dada a hipótese $H_{0}$ ) da variável de decisão $v$ se reduz a 


$$
p_{v \mid H_{0}}(V)=C \sum_{k=0}^{\infty} \delta_{k} \frac{V^{M+k-1}\left(c^{*}\right)^{M+k}}{\Gamma(M+k)} \exp \left(-c^{*} V\right) u(V)
$$

onde

$$
\begin{gathered}
c^{*}=\frac{\beta+2 \sigma_{n}^{2}}{\beta\left(2 \sigma_{n}^{2}\right)^{2}}, \\
C=1,
\end{gathered}
$$

e

$$
\delta_{k}=\left\{\begin{array}{l}
1 ; k=0 \\
0 \quad ; k=1,2, \ldots
\end{array}\right.
$$

uma vez que

$$
\gamma_{k}=0 ; \quad k=1,2,3 \ldots
$$

Finalmente, substituindo (3-169) e (3-170) em (3-167), obtém-se

$$
p_{v \mid H_{0}}(V)=\frac{V^{M-1}\left(c^{*}\right)^{M}}{\Gamma(M)} \exp \left(-c^{*} V\right) u(V)
$$

onde $c^{*}$ é dado por (3-168).

Quando a hipótese $H_{1}$ é verdadeira, a função densidade de probabilidade condicional da variável de decisão, no caso de $s(t)$ com DEP genérica, é dada por (3-126) a (3-131). No caso particular em que $s(t)$ tem DEP plana na faixa de interesse, $\operatorname{com}\left\{\lambda_{i}=1, i=1, \ldots, M\right\}$ (ver (3-161)), a expressão da função densidade de probabilidade condicional (dada a hipótese $H_{1}$ ) da variável de decisão $v$ se reduz a

$$
p_{v \mid H_{1}}(V)=C \sum_{k=0}^{\infty} \delta_{k} \frac{V^{M+k-1}\left(c^{*}\right)^{M+k}}{\Gamma(M+k)} \exp \left(-c^{*} V\right) u(V),
$$

onde

$$
\begin{gathered}
c^{*}=\frac{1}{2 \sigma_{n}^{2} \beta}, \\
C=1,
\end{gathered}
$$

e

uma vez que

$$
\delta_{k}= \begin{cases}1 & ; k=0 \\ 0 & ; k=1,2, \ldots\end{cases}
$$

$$
\gamma_{k}=0 ; \quad k=1,2,3 \ldots
$$

Finalmente, substituindo (3-175) e (3-176) em (3-173), obtém-se

$$
p_{v \mid H_{1}}(V)=\frac{V^{M-1}\left(c^{*}\right)^{M}}{\Gamma(M)} \exp \left(-c^{*} V\right) u(V)
$$

onde $c^{*}$ é dado por (3-174). 


\subsection{3}

\section{Probabilidade de falso alarme e probabilidade de detecção}

A partir da definição da probabilidade de falso alarme, dada por (3-96), e da função densidade de probabilidade condicional da variável de decisão $v$ dada a hipótese $H_{0}$, que no caso particular de $s(t)$ com DEP plana é dada por (3-172) e (3-168), a probabilidade de falso alarme se escreve

$$
P_{f a}=\frac{\left(c^{*}\right)^{M}}{\Gamma(M)} \int_{\eta}^{\infty} V^{M-1} \exp \left(-c^{*} V\right) d V
$$

ou ainda, fazendo-se a mudança de variável de integração $z=c^{*} V$,

$$
P_{f a}=\frac{\left(c^{*}\right)^{M}}{\Gamma(M)} \int_{c^{*} \eta}^{\infty}\left(\frac{Z}{c^{*}}\right)^{M-1} \frac{1}{c^{*}} \exp (-Z) d Z
$$

Após algumas manipulações matemáticas a probabilidade $P_{f a}$ pode ainda ser escrita como

$$
P_{f a}=\frac{\Gamma\left(M, c^{*} \eta\right)}{\Gamma(M)}
$$

com $c^{*}$ é dado por (3-168), ou seja,

$$
c^{*}=\frac{\beta+2 \sigma_{n}^{2}}{\beta\left(2 \sigma_{n}^{2}\right)^{2}},
$$

Em (3-181), $\Gamma(\cdot, \cdot)$ denota a Função Gama Incompleta Superior [35] definida em (3-135) e $\Gamma(\cdot)$ representa a Função Gama [35] definida em (3-136).

De maneira análoga, a partir da definição da probabilidade de detecção, dada por (3-97), e da função densidade de probabilidade condicional da variável de decisão $v$ dada a hipótese $H_{1}$, que no caso particular de $s(t)$ com DEP plana é dada por (3-178) e (3-174), a probabilidade de detecção se escreve

$$
P_{d}=\frac{\left(c^{*}\right)^{M}}{\Gamma(M)} \int_{\eta}^{\infty} V^{M-1} \exp \left(-c^{*} V\right) d V
$$

Novamente, fazendo-se a mudança de variável de integração $z=c^{*} V$ obtém-se, após algumas manipulações matemáticas,

$$
P_{d}=\frac{\Gamma\left(M, c^{*} \eta\right)}{\Gamma(M)}
$$

com $c^{*}$ é dado por (3-174), ou seja

$$
c^{*}=\frac{1}{2 \sigma_{n}^{2} \beta} .
$$

Em (3-184), $\Gamma(\cdot, \cdot)$ denota a Função Gama Incompleta Superior [35] definida em (3-135) e $\Gamma(\cdot)$ representa a Função Gama [35] definida em (3-136). 


\subsection{4}

\section{Estimador da potência média da envoltória complexa do sinal do usuário primário}

Assim como na Subseção 3.8, são consideradas duas possibilidades para a estimação da potência média $\beta$ da envoltória complexa do sinal do usuário primário: estimativa feita a partir da observação das amostras da envoltória complexa do sinal recebido (elementos do vetor $\mathbf{r}$ ) e estimativa feita a partir do valor observado da variável de decisão $v$. Nas seguintes subseções, são abordadas estas duas possibilidades considerando a caracterização particular (definida na Seção 3.9) do sinal produzido pelas transmissões de UPs.

\subsubsection{1}

\section{Estimação a partir do vetor de observações $r$}

No caso particular em que o sinal produzido pelas transmissões dos usuários primários $s(t)$, é caracterizado por um processo estocástico passa-faixa gaussiano ESA, de média nula, com densidade espectral de potência plana na faixa, a matriz covariância do vetor aleatório complexo $\mathbf{s}_{0}$ é uma matriz identidade (ver (3-156)). Portanto, a condição em (3-144), a ser resolvida para a obtenção da equação ML, se reduz a

$$
-M \frac{\partial}{\partial B} \ln \left(B+2 \sigma_{n}^{2}\right)-\mathbf{R}^{\mathrm{H}} \mathbf{R} \frac{\partial}{\partial B}\left(\frac{1}{B+2 \sigma_{n}^{2}}\right)=0
$$

Efetuando-se a derivada em (3-186), obtém-se,

$$
-\frac{M}{B+2 \sigma_{n}^{2}}+\frac{\mathbf{R}^{\mathrm{H}} \mathbf{R}}{\left(B+2 \sigma_{n}^{2}\right)^{2}}
$$

ou ainda

$$
-M\left(B+2 \sigma_{n}^{2}\right)+\mathbf{R}^{\mathrm{H}} \mathbf{R}=0
$$

que deve ser resolvida em relação a $B$. Assim a Equação ML tem uma solução fechada, dada por,

$$
\hat{\beta}_{m l}(\mathbf{R})=\frac{\mathbf{R}^{\mathrm{H}} \mathbf{R}}{M}-2 \sigma_{n}^{2}
$$

Considerando que o resultado da estimativa do parâmetro $\beta$ em (3-189) pode ser negativo, o que é inadequado pois $\beta$ caracteriza potência, propõe-se o estimador subótimo dado por

$$
\hat{\beta}_{2}(\mathbf{R})=\left|\frac{\mathbf{R}^{\mathrm{H}} \mathbf{R}}{M}-2 \sigma_{n}^{2}\right|
$$




\subsubsection{2}

\section{Estimação a partir da observação da variável de decisão $v$}

O DBC precisa estimar o parâmetro $\beta$ com base no valor assumido pela variável de decisão $v$ (definida em (3-163)), que é enviado por cada US.

A partir da expressão do estimador subótimo do parâmetro $\beta$ definido em (3-146) e considerando-se que a função densidade de probabilidade condicional da variável aleatória $v$ (dado $H_{1}$ ), no caso particular em que o sinal $s(t)$ tem densidade espectral de potência plana, é dada por

$$
p_{v \mid \beta=B}(V)=\frac{1}{\left(2 \sigma_{n}^{2} B\right)^{M}} \frac{V^{M-1}}{\Gamma(M)} \exp \left(-\frac{V}{2 \sigma_{n}^{2} B}\right) u(V),
$$

obtém-se

$$
\hat{\beta}_{1}(V)=\max _{B}^{-1}\left[\frac{1}{\left(2 \sigma_{n}^{2} B\right)^{M}} \frac{V^{M-1}}{\Gamma(M)} \exp \left(-\frac{V}{2 \sigma_{n}^{2} B}\right) u(V)\right]
$$

A maximização em (3-192) é obtida resolvendo-se a equação

$$
\frac{d}{d B}\left[\frac{1}{\left(2 \sigma_{n}^{2} B\right)^{M}} \frac{V^{M-1}}{\Gamma(M)} \exp \left(-\frac{V}{2 \sigma_{n}^{2} B}\right)\right]=0 \quad ; \quad V \geq 0
$$

obtendo-se, conforme o desenvolvimento apresentado na Seção B.2 do Apêndice $\mathrm{B}$, a solução

$$
\hat{\beta}_{1}(V)=\frac{V}{M 2 \sigma_{n}^{2}}
$$




\section{4}

\section{Mapas de Ambiente de Rádio}

Conforme definido na descrição do problema, apresentada no Capítulo 2, os terminais HDFSS operando em caráter secundário, executam um sensoriamento de espectro e enviam, para o DBC além das informações sobre sua localização, as informações sobre parâmetros relacionados à presença de transmissões de usuários primários na área de serviço do HDFSS (neste trabalho denominada $S_{T}$ ). Com base nos dados recebidos dos USs cooperadores, o DBC produz uma estimativa da distribuição espacial em relação à interferência gerada pelos UPs que operam na região de interesse. Segundo esta distribuição o DBC produz um Mapa de Ambiente de Rádio que indica, os locais onde o HDFSS pode operar.

Os métodos de geração de REM considerados neste trabalho são apresentados na Seção 4.1. Na Seção 4.2 são definidas métricas que refletem a qualidade dos Mapas de Ambiente de Rádio, e que são utilizadas no Capítulo 5 numa análise comparativa do desempenhos dos dois métodos de geração de REM considerados.

\section{1}

\section{Métodos de Geração dos Mapas de Ambiente de Rádio}

Dois métodos de geração de REM são considerados neste estudo: o método apresentado em [11] (aqui denominado Método_0) e um método original proposto neste trabalho (Método_1). Estes dois métodos são descritos nas subseções 4.1 .1 e 4.1.2, respectivamente.

\subsection{1}

\section{Método_0}

Nesta subseção, o método de geração de REM proposto em [11] é apresentado. Para um melhor entendimento do método, um diagrama em bloco das operações a serem realizadas é apresentado na Figura 4.1. Conforme indicado no diagrama, os $N_{U S}$ usuários secundários operando na área de serviço do HDFSS enviam para o DBC, além das suas localizações $\left\{\left(\theta_{j}, \phi_{j}\right), j=\right.$ $\left.1, \ldots, N_{\mathrm{US}}\right\}$, a informação binária $\left\{d_{j}, j=1, \ldots, N_{\mathrm{US}}\right\}$ sobre suas decisões $\left(d_{j}=1\right.$ se decide-se por $H_{1}$ ou $d_{j}=0$ se decide-se por $\left.H_{0}\right)$. 


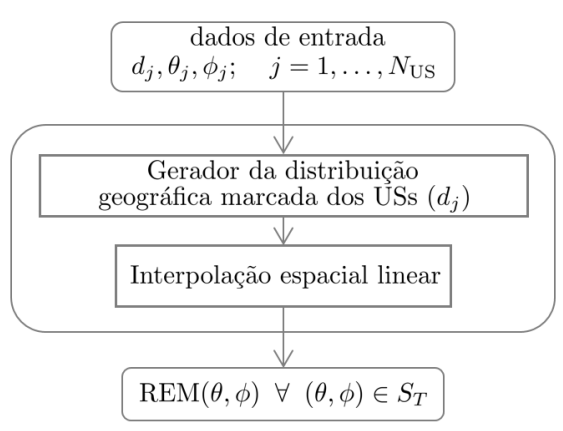

Figura 4.1: Diagrama em blocos do Método_0 de geração do REM

No primeiro passo deste Método_0, a informação recebida pelo DBC é mapeada em uma distribuição geográfica dos USs cooperadores, sendo, cada ponto da distribuição, marcado de acordo com a decisão tomada pelo US correspondente.

Um exemplo desta distribuição é mostrado na Figura 4.2 para $N_{\mathrm{US}}=900$. Na figura os USs que detectaram a presença de transmissões de UP estão marcados em vermelho enquanto os USs que não detectaram a presença de transmissões de UP estão marcados em azul.

No segundo passo deste método, o DBC executa uma interpolação espacial linear sobre os pontos desta distribuição seguido de uma quantização de um nível $(q=1 / 2)$, dando origem ao Mapa de Ambiente de Rádio $\operatorname{REM}(\theta, \phi) \forall(\theta, \phi) \in S_{T}$. (ver exemplo na Figura 4.3).

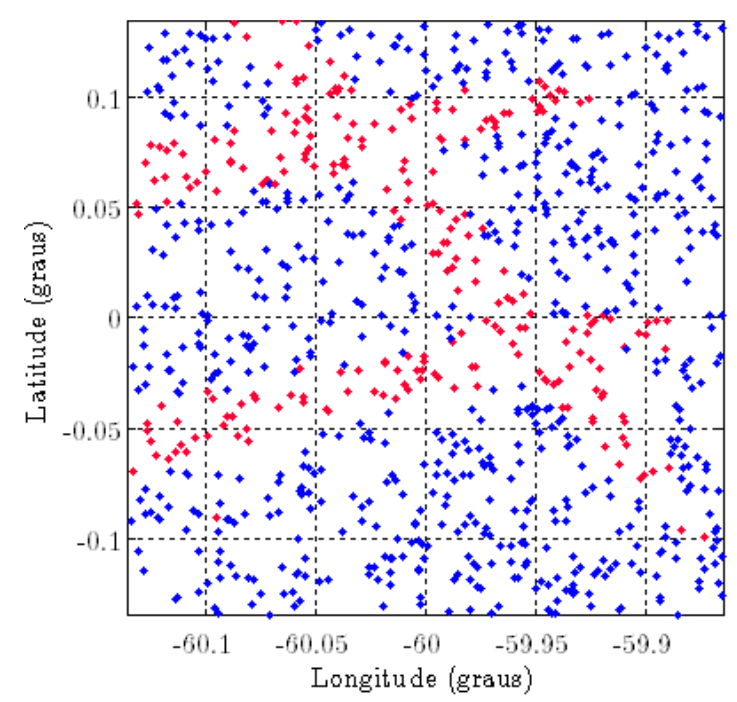

Figura 4.2: Distribuição geográfica marcada dos usuários secundários 


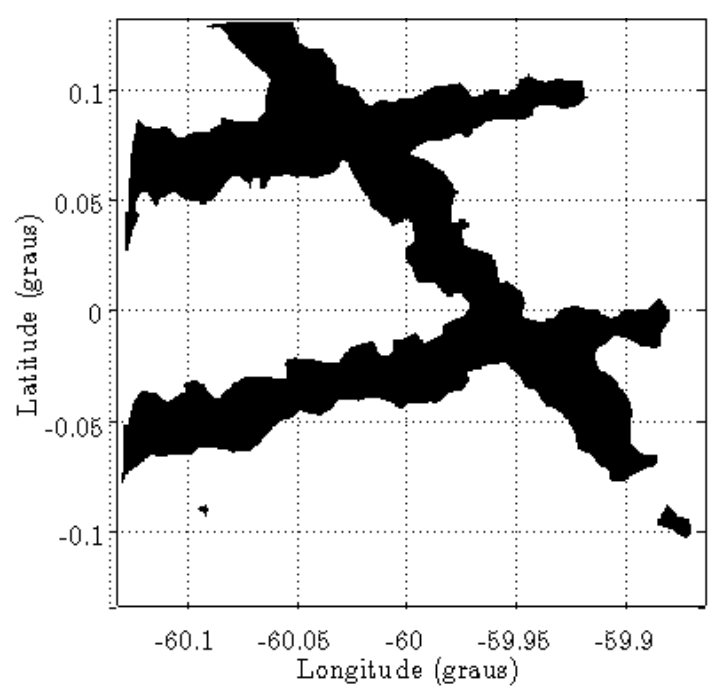

Figura 4.3: Exemplo de resultados para o Método_0 e $N_{\text {US }}=900$

\subsection{2}

\section{Método_1}

O método de geração de REM proposto neste trabalho (aqui denominado Método_1), cujo diagrama em blocos é apresentado na Figura 4.4, ao invés de utilizar a informação binária das decisões dos USs cooperadores, utiliza os valores $\left\{V_{j}, j=1, \ldots, N_{\mathrm{US}}\right\}$ das variáveis de decisão correspondentes a cada um dos USs (variáveis de ponto flutuante).

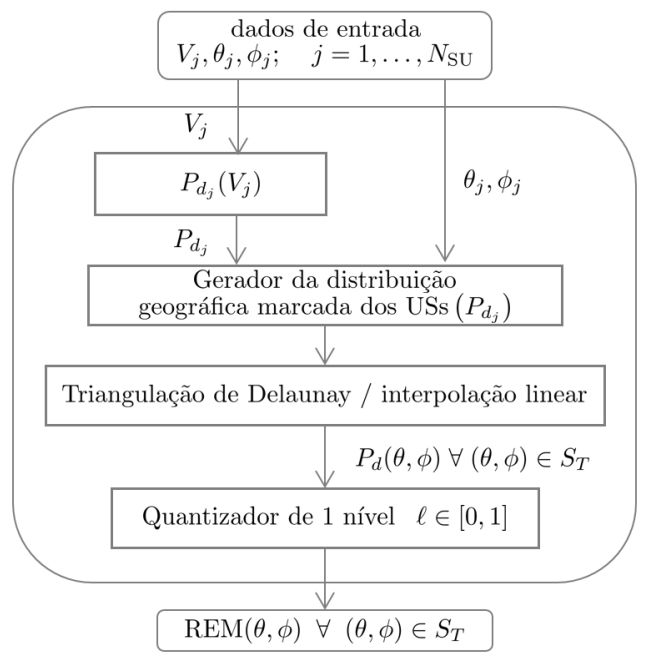

Figura 4.4: Diagrama em blocos do Método_1 de geração do REM

Conforme indicado no diagrama da Figura 4.4, com base nestes valores, é calculada a probabilidade de detecção associada a cada um dos USs. Neste ponto o DBC poderia utilizar uma das duas expressões de $P_{d}$ contidas neste trabalho, a primeira expressão (ver (3-138)) desenvolvida neste trabalho aplica- 
se em casos nos quais o sinal $s(t)$, produzido pelas transmissões de usuários primários, é modelado por um PE passa-faixa gaussiano ESA de média nula com DEP genérica. A segunda expressão (ver (3-184)) deve ser utilizada pelo DBC no caso em que que o sinal $s(t)$, produzido pelas transmissões de usuários primários, é modelado por um PE passa-faixa gaussiano ESA de média nula com DEP plana.

Note que para determinação da probabilidade de detecção a partir do valor assumido pela variável de decisão, feita utilizando-se (3-138) ou (3-184), é necessário o conhecimento do valor da potência média $\beta$ da envoltória complexa dos sinais dos UPs. Este valor é estimado a partir do valor assumido pela variável de decisão $v$. Estimadores de $\beta$ a partir da observação de $v$ foram abordados nas subseções 3.8.2 e 3.9.4.2.

Assim como no Método_0, neste método é também gerada uma distribuição geográfica dos USs, sendo que, neste caso, cada ponto da distribuição é marcado de acordo com o valor da probabilidade de detecção associada a cada um dos USs cooperadores. Um exemplo desta distribuição é mostrado na Figura 4.5 para $N_{\mathrm{US}}=900$.

Através da triangulação de Delaunay [46] e de uma interpolação linear sobre os pontos desta distribuição é obtida uma função contínua $P_{d}(\theta, \phi)$, definida para todos as coordenadas geográficas dentro da região de interesse $(\theta, \phi) \in S_{T}$. Como último passo, um quantizador de um nível $\left(P_{d}=\ell\right)$ é aplicado a esta função, gerando o Mapa de Ambiente de Rádio (ver exemplo na Figura 4.6, obtido com $\ell=0,8)$. 


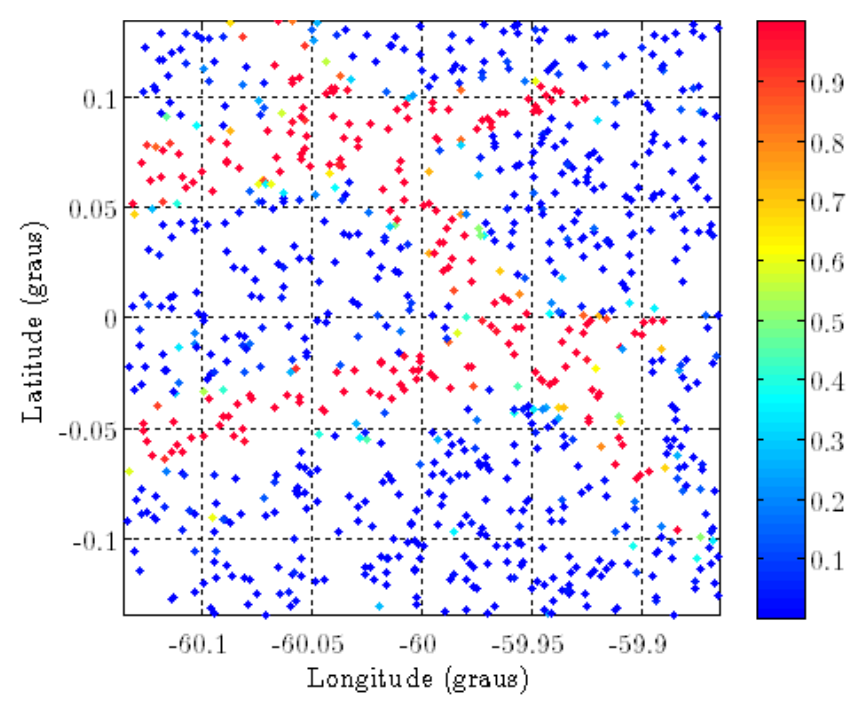

Figura 4.5: Distribuição geográfica marcada dos USs cooperadores

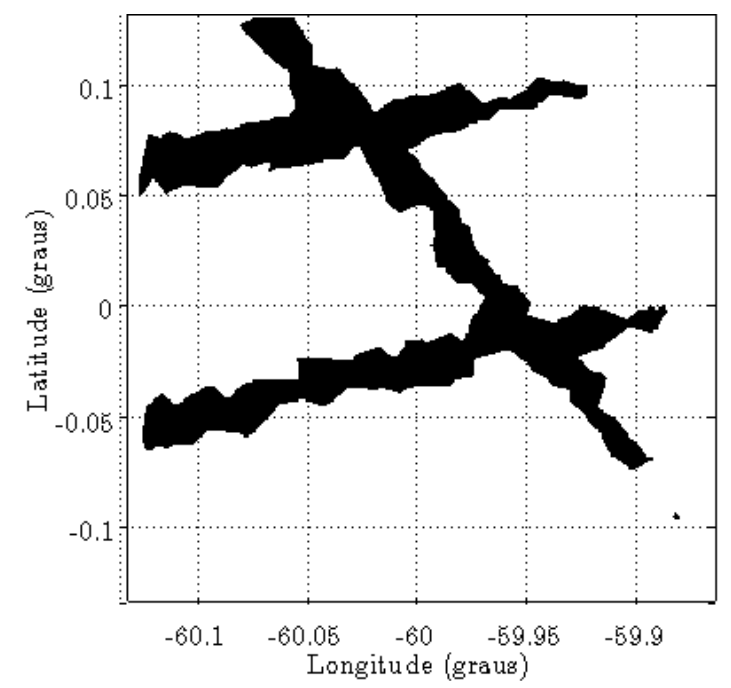

Figura 4.6: Exemplo de resultados para o Método_1 e $N_{\mathrm{US}}=900$ 


\section{2}

\section{Qualidade dos Mapas de Ambiente de Rádio}

Os métodos de geração de REM descritos na Subseção 4.1 podem ser avaliados se aplicados a cenários para os quais seja possível determinar um REM de referência, que pode ser obtido utilizando-se as informações reais dos UPs (e.g. coordenadas geográficas, níveis de densidade de potência de transmissão, diagramas de radiação das antenas envolvidas entre outras).

Considera-se aqui que este REM de referência é definido por

$$
S_{d_{r e f}}=\left\{s \in S_{T}: I \geq I_{\max }\right\}
$$

onde $I_{\max }$ caracteriza o nível máximo de densidade de potência interferente agregada produzida pelos transmissores FS, que um terminal HDFSS pode tolerar na saída de sua antena receptora sem prejuízo para sua operação (critério de proteção definido pelo operador HDFSS).

Uma comparação entre este mapa REM de referência e um mapa REM qualquer, por exemplo, um mapa REM produzido por um dos métodos de geração definidos na Subseção 4.1, permite avaliar a qualidade do mapa produzido. Propõe-se aqui que esta comparação seja feita com base em duas métricas: a Taxa Espacial de Deteç̧ão e a Taxa Espacial de Falso Alarme. Para definir estas métricas considere a Figura 4.7, na qual estão ilustrados um mapa REM de referência $S_{d_{r e f}}$ e um mapa REM $S_{d}$ produzido por um método de geração qualquer, definido por

$$
S_{d}=\left\{s \in S_{T}: \text { US não pode operar }\right\}
$$

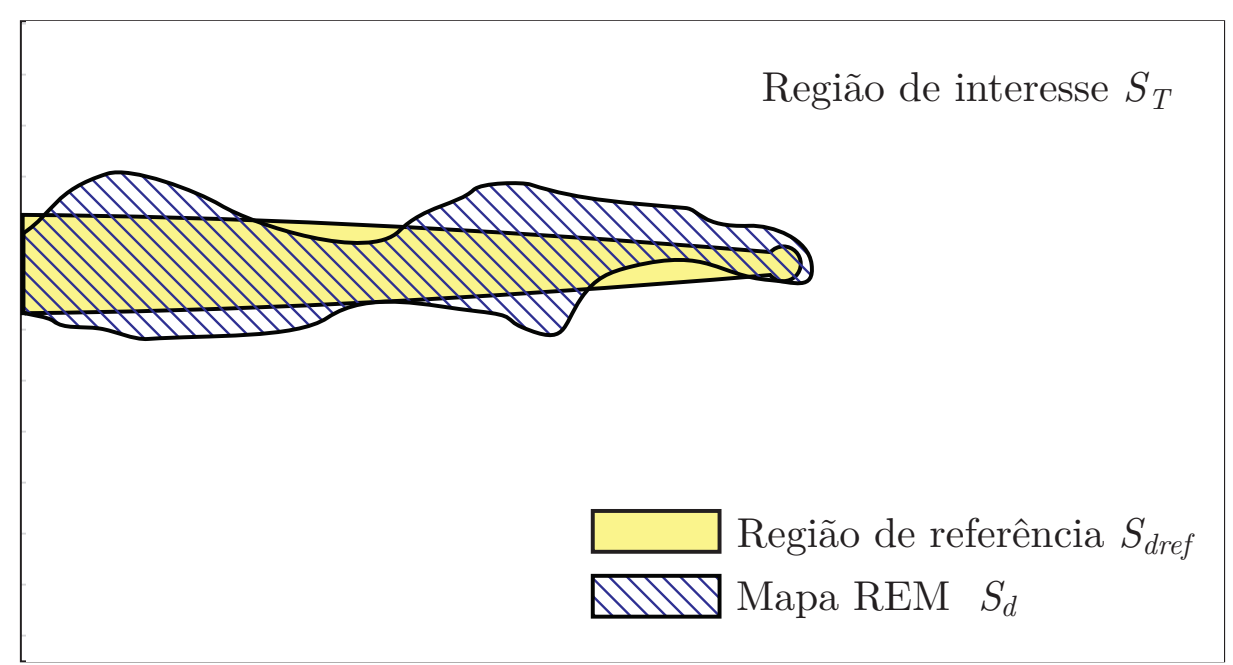

Figura 4.7: Mapas REM de referência e mapa REM gerado 
Na Figura 4.7, $S_{T}$ representa a região de interesse, na qual o HDFSS e o FS compartilham a mesma faixa de frequências (área de serviço do HDFSS). Com base nesta figura, a Taxa espacial de detecção é então, definida por

$$
T_{d}=\frac{A\left(S_{d} \cap S_{d_{r e f}}\right)}{A\left(S_{d_{r e f}}\right)}
$$

onde $A(\cdot)$ denota o operador que define a área da região indicada no seu argumento, e a Taxa de Falso alarme é dada por

$$
T_{f a}=\frac{A\left(S_{d} \cap \overline{S_{d_{r e f}}}\right)}{A\left(\overline{S_{d_{r e f}}}\right)}
$$

onde $\overline{S_{d_{r e f}}}$ representa o complemento de $S_{d_{r e f}}$ em relação a $S_{T}$.

A qualidade do REM pode então ser visualizada por meio de um gráfico $\left(T_{d} \times T_{f a}\right)$, do tipo ROC (Receiver Operating Characteristic) [47]. Neste tipo de gráfico, o REM ideal corresponde a $T_{d}=1$ e $T_{f a}=0$. 


\section{5 \\ Resultados Numéricos}

Os cenários analisados neste capitulo envolvem enlaces do Serviço Fixo Terrestre, na qualidade de usuários primários, operando na Faixa 18,1-19,7 GHz e enlaces do HDFSS, operando em carácter secundário, na mesma faixa, na direção espaço-terra. O satélite, localizado na órbita de satélites geoestacionários (GEO), se comunica com os terminais (estações terrenas de pequeno porte) localizados na área de serviço $S_{T}$ do HDFSS, transmitindo na mesma faixa de frequências do usuário primário. A Figura 5.1 ilustra esta situação de compartilhamento de frequências entre os dois sistemas.

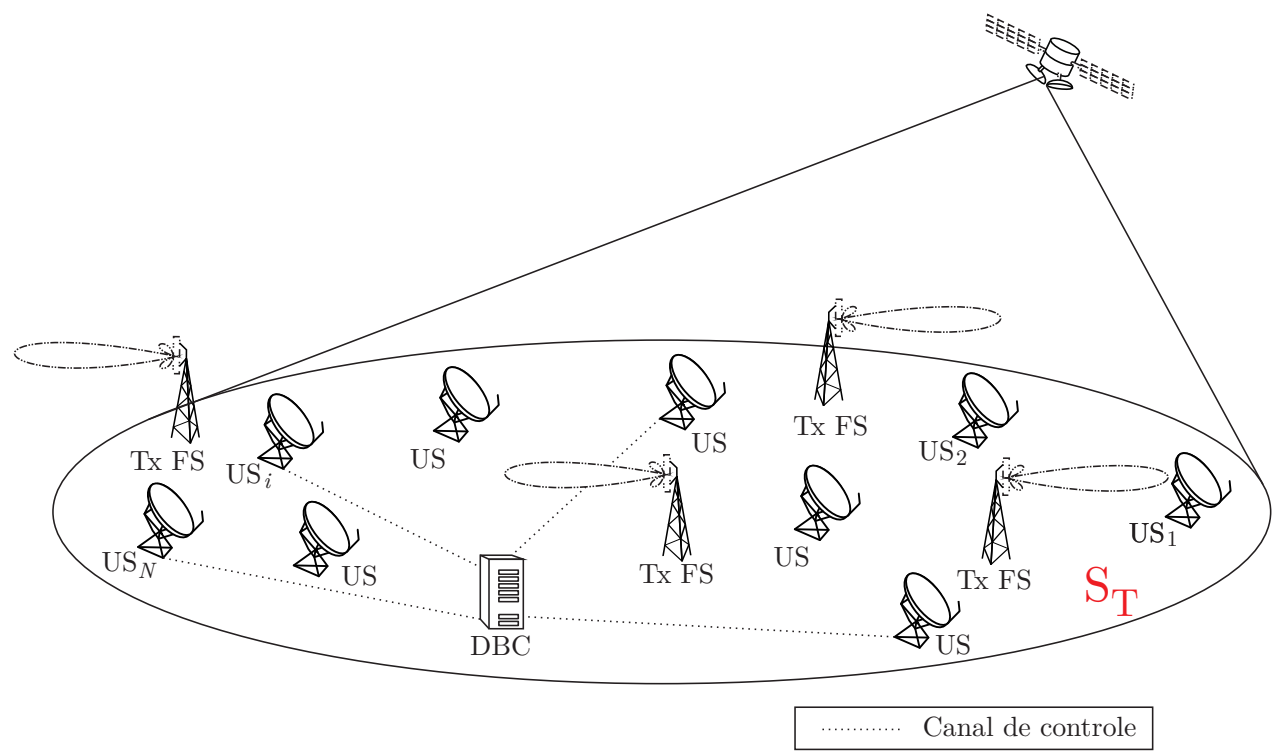

Figura 5.1: Enlaces do Serviço Fixo Terrestre e enlaces do HDFSS em um cenário de compartilhamento de frequências

Os parâmetros técnicos dos sistemas considerados no estudo são apresentados nas seções 5.1 e 5.2. O esquema de simulação utilizado neste trabalho é detalhado na Seção 5.3.

Os dois métodos de geração de REM descritos na Seção 4.1 são avaliados em dois diferentes cenários. O primeiro cenário considera a presença de apenas um enlace do Serviço Fixo Terrestre e de vários enlaces do HDFSS localizados na área de interesse, os resultados obtidos para este cenário são apresentados na Subseção 5.4.1. O segundo cenário considera a presença de três enlaces do 
Serviço Fixo Terrestre e de vários enlaces do HDFSS localizados na área de interesse, os resultados obtidos para este cenário são apresentados na Subseção 5.4 .2 .

\section{1}

Parâmetros técnicos dos sistemas do Serviço Fixo Terrestre (usuários primários)

Os transmissores e receptores dos enlaces FS considerados neste trabalho operam na frequência de $18,7 \mathrm{GHz}$ e estão colocados a uma altura de 29 metros. A densidade de potência transmitida em cada um dos enlaces é de -20 $\mathrm{dB}(\mathrm{W} / \mathrm{MHz})$. Para as antenas transmissoras do FS, considerou-se um ganho máximo do lóbulo principal de $40 \mathrm{dBi}$ (com uma eficiência 0.55) e o diagrama de radiação dado pela Recomendação ITU-R F.699 [48]. Este diagrama de radiação está ilustrado na Figura 5.2.

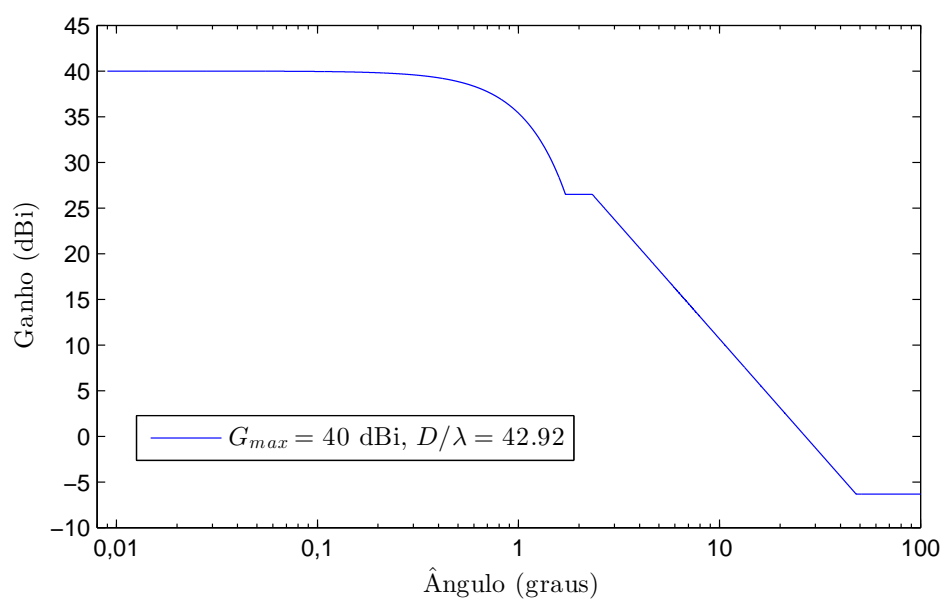

Figura 5.2: Diagrama de radiação da antena transmissora do FS

Nos cenários avaliados neste capítulo, duas densidades espectrais de potência são consideradas para a envoltória complexa dos sinais transmitidos pelos usuários primários: densidade espectral de potência com dois polos e densidade espectral de potência plana. Os parágrafos a seguir apresentam as características destas duas densidades espectrais de potência.

Densidade Espectral de Potência com 2 polos: Neste caso a densidade espectral de potência da envoltória complexa $\tilde{s}(t)$, dada por (3-43), se escreve

$$
S_{\tilde{s}}(f)=\frac{2 a \beta}{a^{2}+\left(2 \pi\left(f-f_{d}\right)\right)^{2}}
$$


onde $f_{d}$ é um deslocamento em frequência conhecido, neste trabalho tomado igual a $f_{d}=0,2 \mathrm{MHz}$ e $a$ é um parâmetro relacionado à largura de banda da envoltória complexa $\tilde{s}(t)$.

Como $\tilde{s}(t)$ é um processo estocástico ESA, sua função autocorrelação é dada por $R_{\tilde{s}}(\tau)=\mathcal{F}^{-1}\left[S_{\tilde{s}}(f)\right]$, ou seja,

$$
R_{\tilde{s}}(\tau)=\beta \exp ^{-a|\tau|} \cos \left(2 \pi f_{d} \tau\right)+j \beta \exp ^{-a|\tau|} \operatorname{sen}\left(2 \pi f_{d} \tau\right)
$$

sendo sua potência média dada por

$$
P_{\tilde{s}}=R_{\tilde{s}}(0)=\beta
$$

O módulo e a fase desta função autocorrelação estão ilustrados na Figura 5.3 para $a=2,4725 \times 10^{5} \mathrm{~Hz}$ e $f_{d}=0,2 \mathrm{MHz}$.

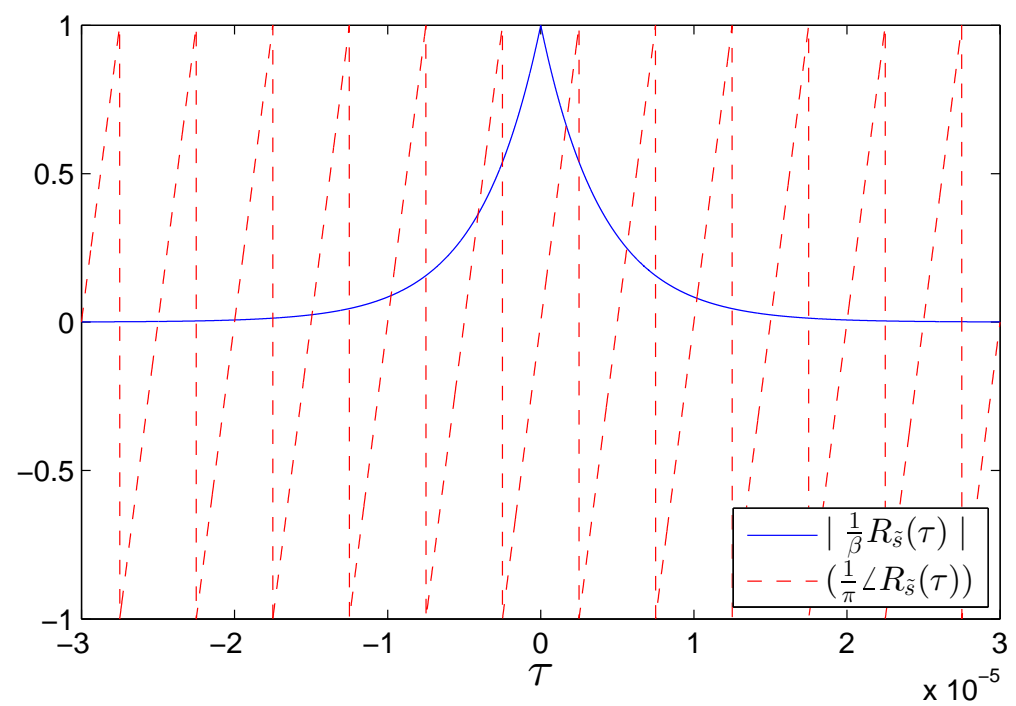

Figura 5.3: Módulo e fase da função autocorrelação $R_{\tilde{s}}(\tau)\left(a=2,4725 \times 10^{5} \mathrm{~Hz}\right.$ e $f_{d}=0,2 \mathrm{MHz}$ ).

Considerou-se ainda que a banda $W$ de $95 \%$ desta envoltória complexa (banda que retém 95\% de sua potência média) é igual a $1 \mathrm{MHz}$. Neste caso, o parâmetro $a$ em (5-1) é determinado resolvendo-se a equação

$$
\int_{0}^{W / 2} \frac{2 a \beta}{a^{2}+(2 \pi F)^{2}} d F=0,95 \beta
$$

Densidade Espectral de Potência plana : Neste caso, a densidade espectral de potência da envoltória complexa de $s(t)$, dada por (3-43), se escreve

$$
S_{\tilde{s}}(f)=\beta S_{\tilde{s}_{0}}(f)
$$

sendo

$$
S_{\tilde{s}_{0}}(f)=\frac{1}{W} \operatorname{ret}_{W}(f) \text {. }
$$


A função autocorrelação da envoltória complexa $\tilde{s}(t)$, definida em (3-45), se escreve

$$
R_{\tilde{s}}(\tau)=\beta R_{\widetilde{s}_{0}}(\tau)
$$

onde

$$
R_{\tilde{s}_{0}}(\tau)=\operatorname{sinc}(W \tau)
$$

com a função $\operatorname{sinc}(\cdot)$ definida em (3-23). Note que, a potência média da envoltória complexa $\tilde{s}(t)$ se escreve, neste caso

$$
P_{\tilde{s}}=R_{\tilde{s}}(0)=\beta R_{\tilde{s}_{0}}(0)=\beta
$$

\section{2 \\ Parâmetros técnicos dos enlaces HDFSS (usuários secundários)}

Conforme mencionado anteriormente, a análise pretendida envolve a interferência produzida pelas transmissões dos usuários primários nos terminais HDFSS, uma vez que estas interferências podem vir a inviabilizar a operação do lance de descida do enlace HDFSS no canal desejado.

Considera-se neste trabalho que a área de serviço do HDFSS, é definida como uma região quadrada de $30 \mathrm{~km}$ de lado, centrada nas coordenadas geográficas $\left(60^{\circ} \mathrm{O}, 0^{\circ}\right)$.

As localizações geográficas dos terminais HDFSS (usuários secundários) em sua área de serviço foram modeladas por um Processo Pontual de Poisson (PPP) [49] homogêneo, bidimensional, com densidade $\lambda_{p p p}$ pontos $/ \mathrm{km}^{2}$. Foram obtidos resultados para valores de $\lambda_{p p p}$ iguais a $0.5,1.0$ e 1.5 pontos $/ \mathrm{km}^{2}$ (o que corresponde, em média, a 450, 900 e 1350 usuários secundários na área de serviço $S_{T}$ do HDFSS, respectivamente).

Uma amostra do Processo Pontual de Poisson bidimensional utilizado na caracterização das posições geográficas dos usuários secundários é ilustrada na Figura 5.4 para o caso de $\lambda_{p p p}=1$ pontos $/ \mathrm{km}^{2}$ (número médio de USs na área de serviço $N_{U S}=900$ ), na Figura 5.4 o contorno em verde indica a fronteira da área de serviço do HDFSS.

Para todos os terminais HDFSS foi considerada uma altura de 9 metros, um nível de densidade de ruído térmico de $-140 \mathrm{~dB}(\mathrm{~W} / \mathrm{MHz})$ e uma antena receptora de ganho máximo de $42 \mathrm{dBi}$ (eficiência 0,55 ) e apontada para um satélite localizado na órbita de satélites geoestacionários, na posição orbital $15,607^{\circ} \mathrm{O}$. Também neste caso, o diagrama de radiação da Recomendação ITUR F.699 [48] foi considerado para estas antenas. Este diagrama está ilustrado na Figura 5.5. 


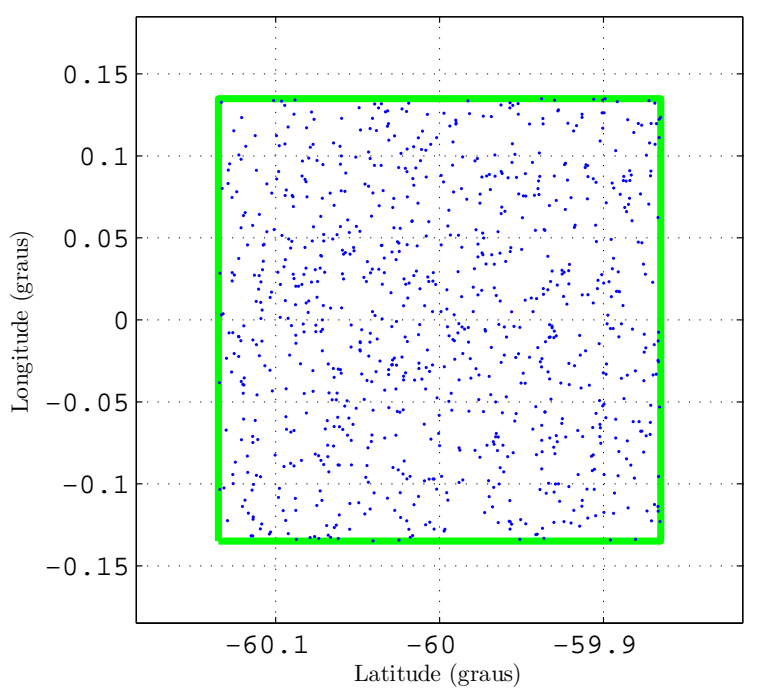

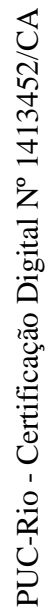

Figura 5.4: Distribuição dos USs na região de interesse para $\lambda_{p p p}=$ 1 pontos $/ \mathrm{km}^{2}$

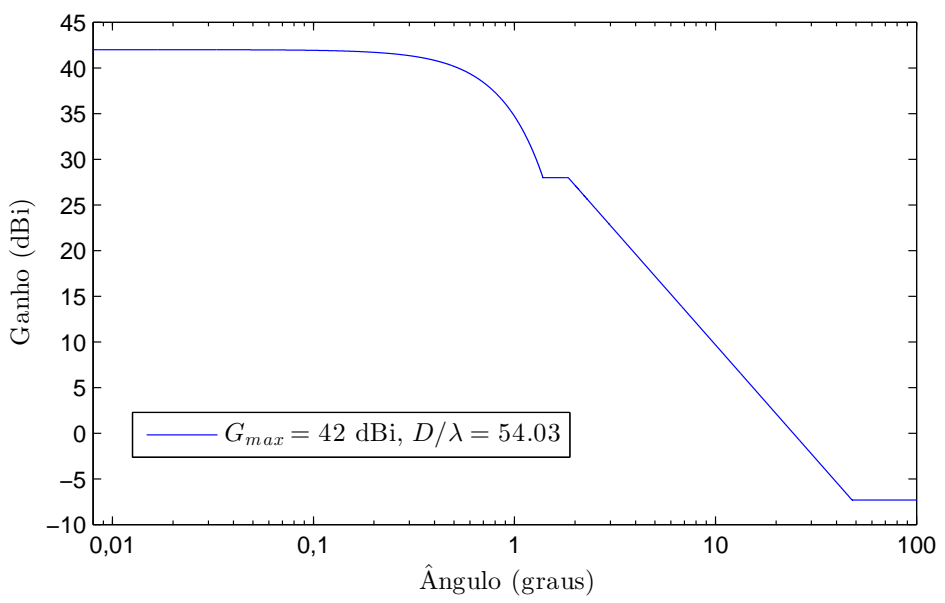

Figura 5.5: Diagrama de radiação da antena receptora do terminal HDFSS 


\section{3 \\ Esquema de Simulação}

Na Figura 5.6 é apresentado o esquema de simulação utilizado na obtenção de resultados que refletem os desempenhos dos métodos de geração de REM considerados neste trabalho (Método_0 e Método_1).

Neste esquema, a partir da especificação da densidade média de pontos do Processo Pontual de Poisson homogêneo [49], é gerada uma amostra do processo, resultando em um determinado número de usuários secundários $N_{U S}$ e em suas coordenadas geográficas $\left\{\left(\theta_{j}, \phi_{j}\right), j=1, \ldots, N_{U S}\right\}$.

Uma vez conhecidas as localizações dos usuários secundários na região de interesse $S_{T}$, e considerando-se os parâmetros técnicos dos enlaces do Serviço Fixo Terrestre e dos enlaces HDFSS, previamente definidos nas seções 5.1 e 5.2, é feito o cálculo do nível médio da densidade de potência interferente agregada atingindo cada um dos $N_{U S}$ usuários secundários.

Com base nestes níveis de potência interferente, são geradas, considerando-se a caracterização em (3-62), $M$ amostras da envoltória complexa do sinal observado por cada um dos usuários secundários, ou seja, são gerados os vetores $\left\{\mathbf{r}_{j}, j=1, \ldots, N_{U S}\right\}$.

Num próximo passo são elaborados os cálculos executados por cada um dos US. Inicialmente é estimado o valor da potência média $\beta$ da envoltória complexa do agregado de sinais produzido pelos UPs. Devido à complexidade envolvida na implementação do estimador de máxima verossimilhança no caso de sinais de UPs com DEP genérica, propõe-se, neste trabalho, que seja utilizada pelos USs, o estimador subótimo dado por (3-190). Conforme indicado na Subeção B.3.1 do Apêndice B, o efeito da utilização deste estimador subótimo é bem pequeno. Note que a estimativa do valor de $\beta$ é necessária para o cálculo, por cada um dos USs, dos valores $\left\{D_{j}, j=1, \ldots, N_{U S}\right\}$ e $\left\{V_{j}, j=1, \ldots, N_{U S}\right\}$ (conforme (3-93) ou (3-163)) assumidos pelas variáveis que caracterizam as informações binárias das decisões tomadas na detecção e pelas variáveis de decisão, respectivamente. Estes valores alimentam, juntamente com as posições geográficas dos usuários secundários, o Método de Geração de REM em análise. Note que, conforme indicado nas subseções 4.1.1 e 4.1.2, o primeiro conjunto de valores é utilizado pelo Método_0 de geração de REM e o segundo pelo Método_1.

Cada um dos dois Mapas de Ambiente de Rádio obtidos (um deles pelo Método_0 e o outro pelo Método_1) é comparado ao REM de referência, resultando em valores específicos para a Taxa Espacial de Falso Alarme $T_{f a}$ e para a Taxa Espacial de Detecção $T_{d}$. Em cada uma das situações consideradas, o procedimento descrito acima foi repetido 5.000 vezes gerando, para cada 
método de geração de REM, um conjunto de 5000 pares $\left(T_{f a}, T_{d}\right)$.

É importante ressaltar que, no caso do Método_1, foram considerados 4 diferentes valores do nível de quantização $\ell$.

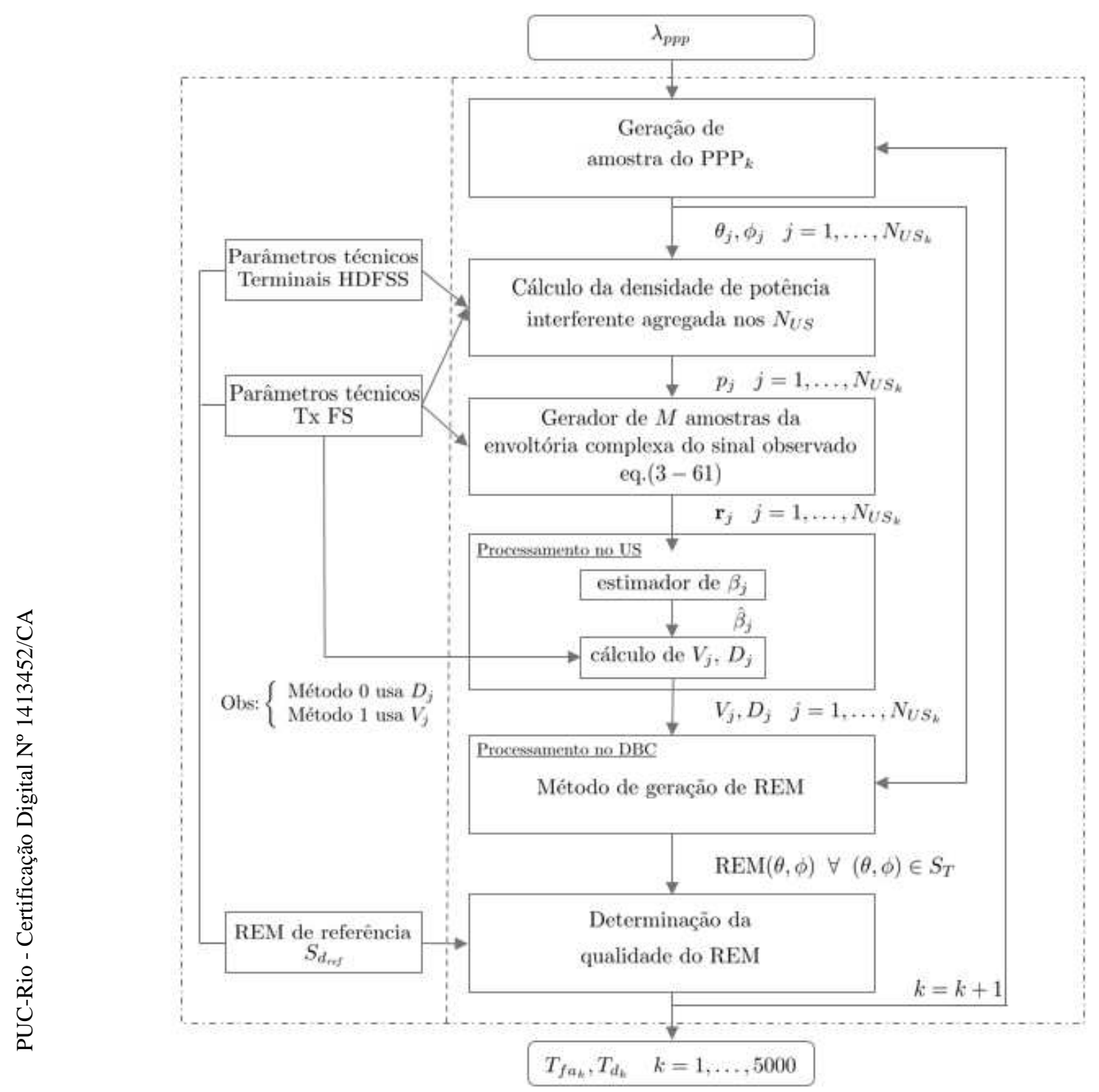

Figura 5.6: Esquema de simulação utilizado na obtenção das métricas de desempenho dos métodos de geração de REM avaliados 


\section{4 \\ Desempenho dos métodos de geração de REM}

O esquema de simulação apresentado na Figura 5.6 foi utilizado na obtenção dos pares $\left(T_{f a}, T_{d}\right)$, que refletem os desempenhos dos métodos de geração de REM considerados neste trabalho (Método_0 e Método_1). Estes resultados permitem uma comparação do desempenho destes dois métodos, além de fornecerem subsídios, através de curvas do tipo ROC, para a escolha do parâmetro $\ell$ (nível de quantização) utilizado pelo Método_1.

Dois cenários são analisados nesta seção: o primeiro deles envolve apenas um enlace FS (aqui denominado Cenário 1) e o segundo três enlaces FS na área de serviço do HDFSS (aqui denominado Cenário 2). Em ambos os cenários os terminais HDFSS, que operam como usuários secundários, estão distribuídos geograficamente de acordo com um Processo Pontual de Poisson, homogêneo e com densidade $\lambda_{p p p}$ pontos $/ \mathrm{km}^{2}$. As análises destes cenários são feitas nas seções 5.4.1 e 5.4.2, respectivamente.

Em cada um dos cenários, três diferentes casos são analisados. O primeiro caso considera que o sinal produzido pelas transmissões dos usuários primários $s(t)$, é caracterizado por um processo estocástico passa-faixa gaussiano ESA, de média nula, com densidade espectral de potência de dois polos, sendo a densidade espectral de potência de sua envoltória complexa dada por (5-1). O segundo caso considera que o sinal produzido pelas transmissões dos usuários primários $s(t)$, é caracterizado por um processo estocástico passafaixa gaussiano ESA, de média nula, com densidade espectral de potência plana, sendo a densidade espectral de potência de sua envoltória complexa dada por (5-5).

No terceiro caso, analisa-se uma situação onde, embora o sinal produzido pelas transmissões dos usuários primários tenha uma densidade espectral de potência de dois polos, o Centro de Base de Dados gera o Mapa de Ambiente de Rádio considerando que a densidade espectral de potência dos sinais transmitidos pelos usuários primários é plana. Neste terceiro caso, é possível analisar as possíveis variações, nos desempenhos dos métodos analisados, proporcionada pelo conhecimento da densidade espectral de potência dos sinais dos usuários primários.

Conforme já indicado, em todos os cenários foram consideradas três densidades de pontos para o PPPs: $\lambda_{p p p}=0,5, \lambda_{p p p}=1,0$ e $\lambda_{p p p}=$ 1,5 pontos $/ \mathrm{km}^{2}$, ou seja, números médios de USs respectivamente iguais a 450, 900 e 1350 US na área de serviço $S_{T}$ do HDFSS. 


\subsection{1}

\section{Cenário 1: apenas um usuário primário operando na região de interesse}

Neste cenário considera-se a presença de um usuário primário (FS) na região de interesse $S_{T}$, as localizações geográficas consideradas para o transmissor e o receptor do enlace do Serviço Fixo Terrestre são apresentadas na Tabela 5.1. O percurso do enlace FS é ilustrado na Figura 5.7 onde o contorno em verde indica a fronteira da área de serviço do HDFSS.

Tabela 5.1: Coordenadas do transmissor e receptor do enlace FS considerado.

\begin{tabular}{|c|c|c|c|c|}
\hline UP & Long do Tx & Lat do Tx & Lon do Rx & Lat do Rx \\
\hline 1 & $60^{\circ} \mathrm{O}$ & $0^{\circ}$ & $60,1349^{\circ} \mathrm{O}$ & $0^{\circ}$ \\
\hline
\end{tabular}

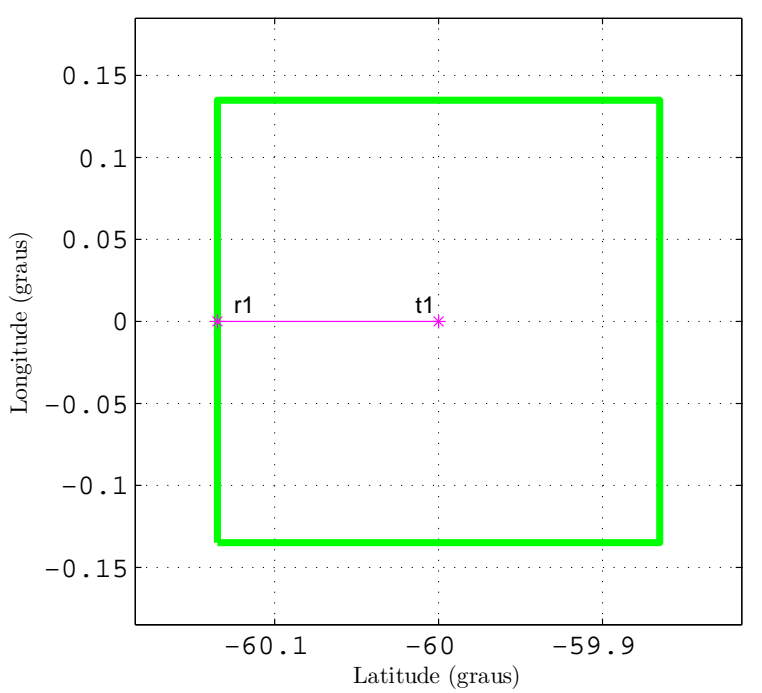

Figura 5.7: Localização do UP na região de interesse

A partir dos parâmetros técnicos considerados para os enlaces FS e HDFSS, a densidade de potência interferente agregada produzida pelo transmissor FS na saída da antena receptora de um terminal HDFSS foi determinada para todas as possíveis posições do terminal HDFSS dentro de sua área de serviço $S_{T}$. O resultado obtido é apresentado na Figura 5.8.

Note que este resultado é importante para a determinação do Mapa de Ambiente de Rádio de referência $\left(S_{d_{r e f}}\right)$, definido como a região da área de serviço $S_{T}$ onde a densidade de potência interferente agregada produzida pelos transmissores FS na saída da antena de um terminal HDFSS é superior ou igual a um valor máximo tolerável $I_{\max }$ de interferência. A Figura 5.9 apresenta o REM de referência obtido a partir da Figura 5.8 considerando-se um nível de $I_{\max }=-139 \mathrm{~dB}(\mathrm{~W} / \mathrm{MHz})$. Este valor de $I_{\max }$ é sugerido pela Recomendação 
ITU-R F.758 [50] para utilização em estudos de compartilhamento envolvendo o Serviço Fixo Terrestre e outros serviços.

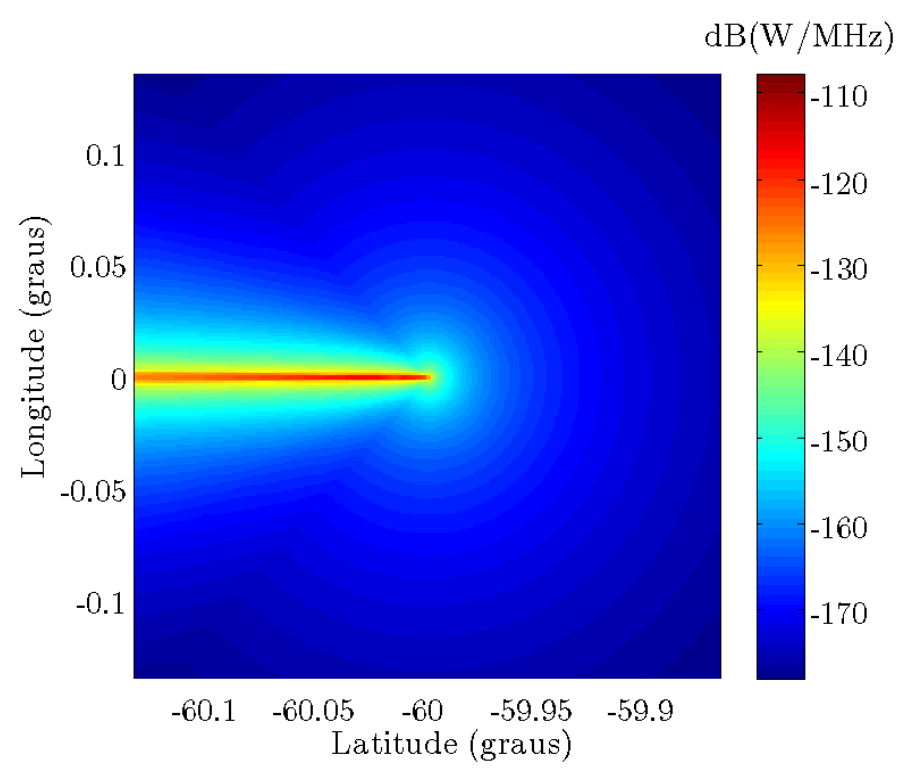

Figura 5.8: Densidade de potência interferente agregada produzida pelo transmissor FS na saída da antena receptora do terminal HDFSS em função da sua localização.

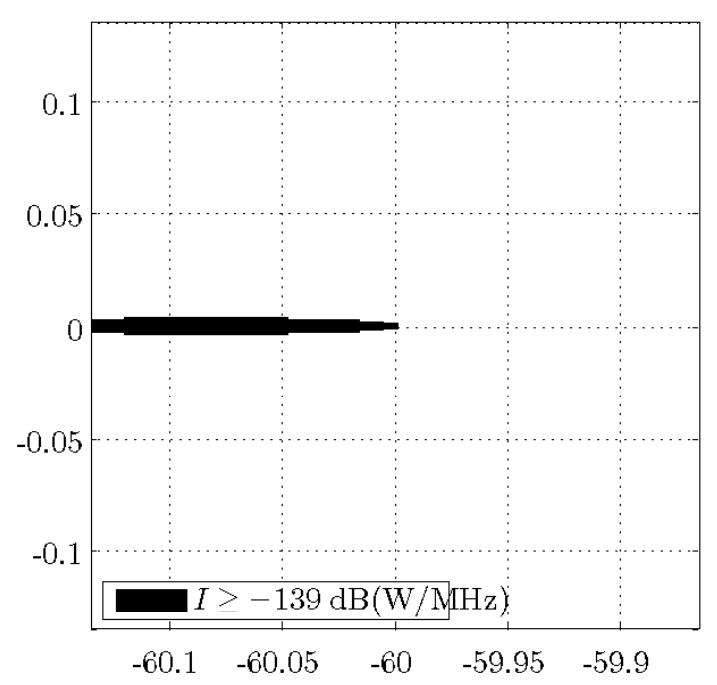

Figura 5.9: Mapa de Ambiente de Rádio de referência $\left(S_{d_{r e f}}\right)$ para um nível de $I_{\max }=-139 \mathrm{~dB}(\mathrm{~W} / \mathrm{MHz})$.

Considerando-se este primeiro cenário, os resultados relativos aos três casos identificados no início da Seção 5.4 são apresentados a seguir. 
Cenário 1, Caso 1: usuário primário com DEP de 2 polos Os valores dos pares $\left(T_{f a}, T_{d}\right)$ obtidos nos casos de $\lambda_{p p p}=0,5, \lambda_{p p p}=1,0$ e $\lambda_{p p p}=$ 1,5 pontos $/ \mathrm{km}^{2}$ (5000 valores para cada método analisado) são apresentados nas figuras 5.10, 5.11 e 5.12, respectivamente.

Nestas figuras são ainda mostrados, através de asteriscos, os pontos médios $\left(\bar{T}_{f a}, \bar{T}_{d}\right)$ associados ao método Método_1, além da curva do tipo ROC $\left(\bar{T}_{f a}\right.$ versus $\left.\bar{T}_{d}\right)$ que passa por estes pontos. Para efeito de comparação, o ponto médio associado ao Método_0 é também indicado na figura, através de um triangulo. Note que estas curvas são importantes para a escolha do nível de quantização $\ell$ a ser utilizado no Método_1 de geração de REM, permitindo a escolha do nível de quantização mais adequado.

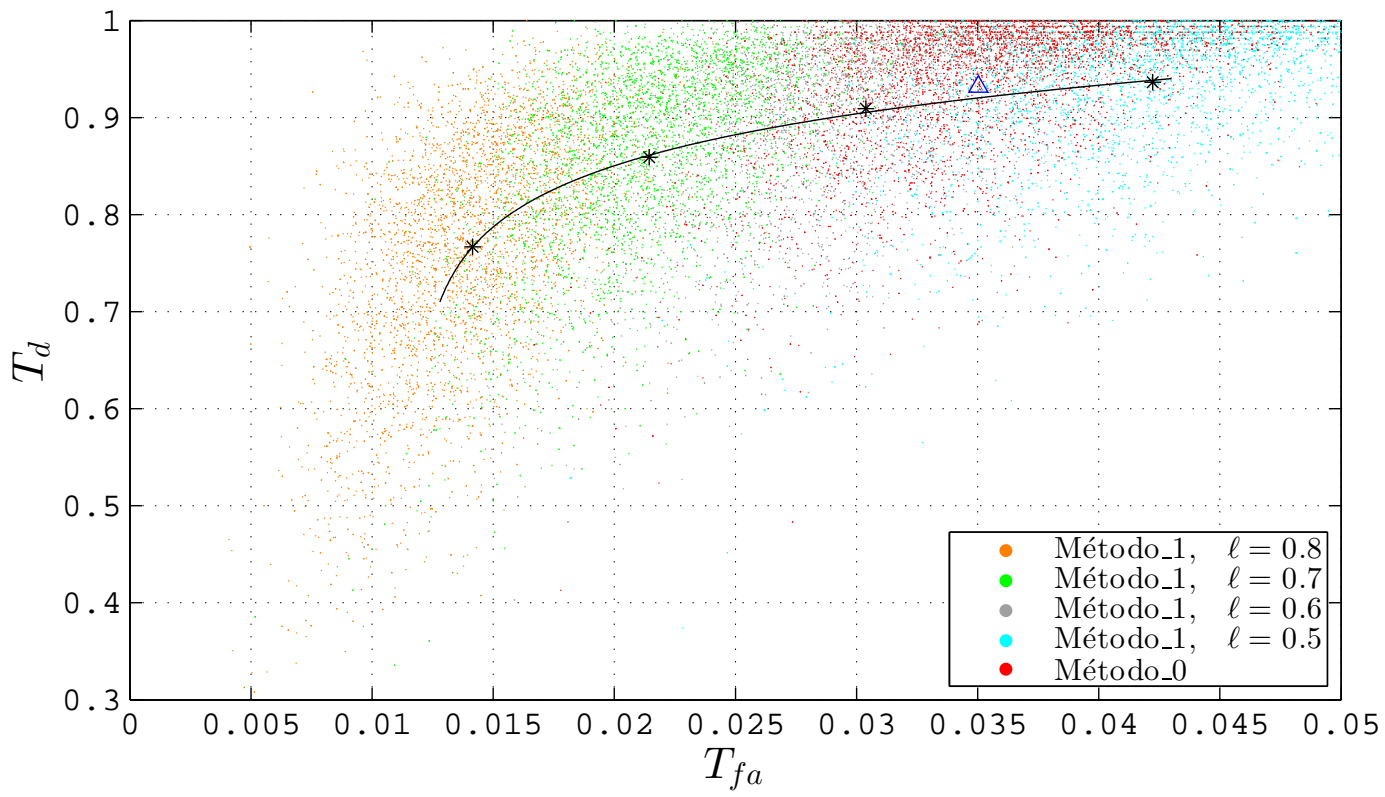

Figura 5.10: Pares $\left(T_{f a}, T_{d}\right)$ obtidos para o Cenário 1 , Caso $1 \mathrm{com} \lambda_{p p p}=$ 0,5 pontos $/ \mathrm{km}^{2}$

O efeito produzido por variações na densidade média de usuários secundários pode ser observada na Figura 5.13, onde são presentadas as curvas do $\bar{T}_{f a}$ versus $\bar{T}_{d}$ associadas a $\lambda_{p p p}=0,5, \lambda_{p p p}=1,0$ e $\lambda_{p p p}=1,5$ pontos $/ \mathrm{km}^{2}$.

As curvas na Figura 5.13 mostram que, conforme o número médio de usuários secundários presentes na região de interesse aumenta, o desempenho dos métodos de geração de REM melhora. Note que, por exemplo, para um valor de $\bar{T}_{f a}=0,02$ o Método_1 apresenta um aumento na $\bar{T}_{d}$ de 0,85 para 0,95 quando a densidade de pontos $\lambda_{\text {ppp }}$ aumenta de 0,5 para 1,5 .

Com base nas figuras 5.10, 5.11 e 5.12 pode-se observar que, em ambos os métodos de geração de REM, a variância dos pontos $\left(T_{f a}, T_{d}\right)$, refletida no tamanho das nuvens de pontos obtidas, diminui conforme a densidade de 


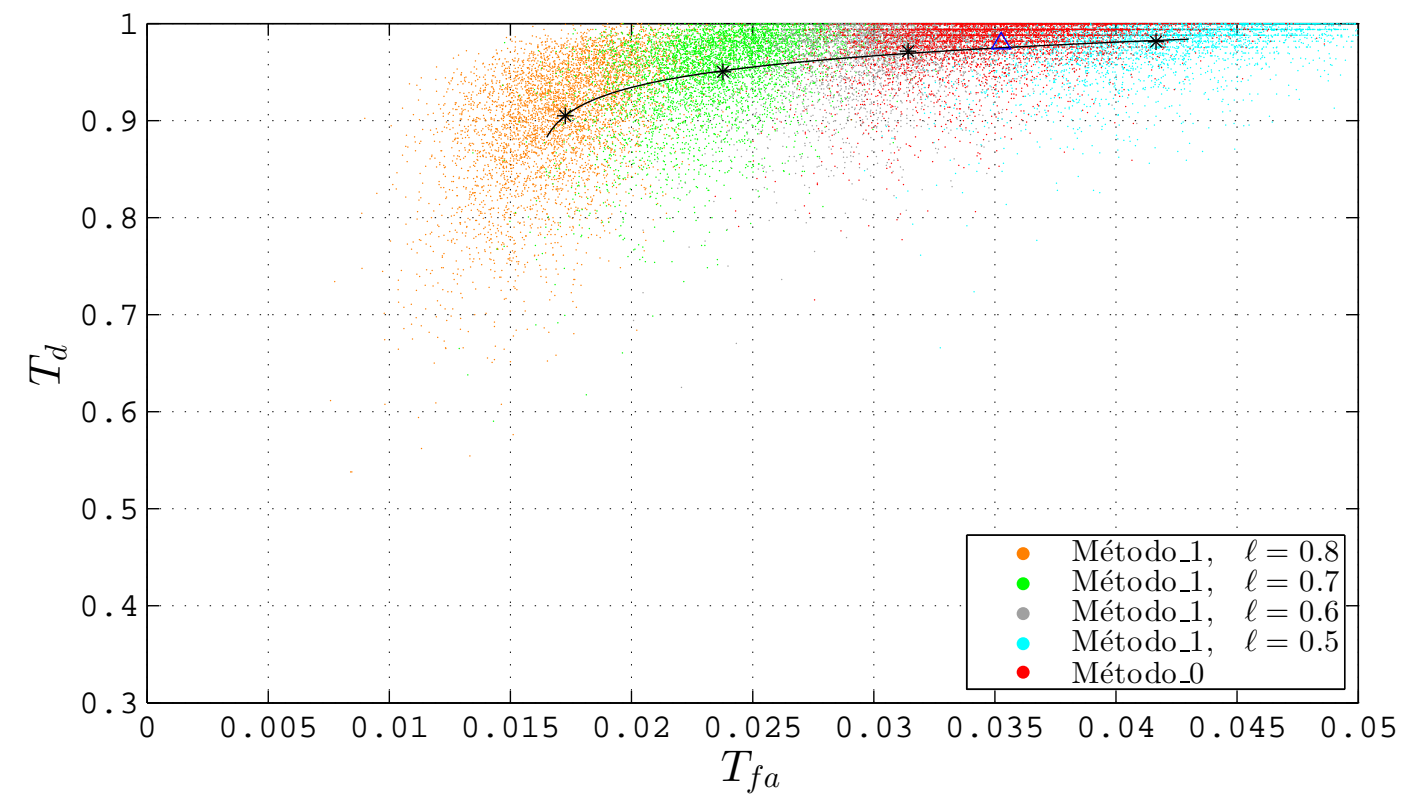

Figura 5.11: Pares $\left(T_{f a}, T_{d}\right)$ obtidos para o Cenário 1, Caso 1 com $\lambda_{p p p}=$ 1 pontos $/ \mathrm{km}^{2}$

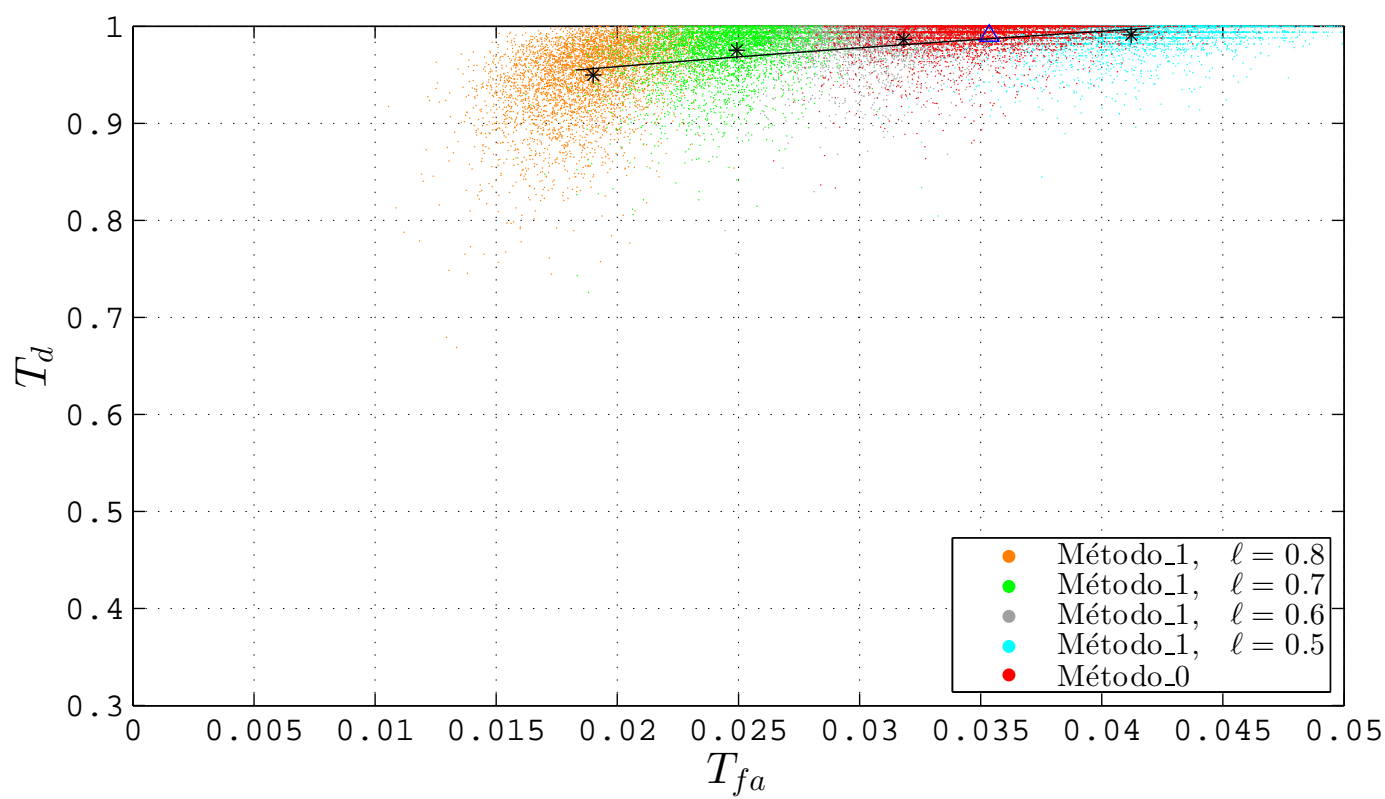

Figura 5.12: Pares $\left(T_{f a}, T_{d}\right)$ obtidos para o Cenário 1, Caso 1 com $\lambda_{p p p}=$ 1,5 pontos $/ \mathrm{km}^{2}$

usuários secundários na região de interesse aumenta. Isto acontece porque a precisão dos mapas REM gerados aumenta com o número de USs que estejam cooperando. Note que a partir destas curvas é possível determinar, conhecido o valor da densidade de pontos $\lambda_{p p p}$, o valor do nível de quantização $\ell$ mais adequado para o ponto de operação desejado $\left(\bar{T}_{f a}, \bar{T}_{d}\right)$. Um outro ponto observado é que, em todos os casos, o desempenho do Método_0 é bastante 


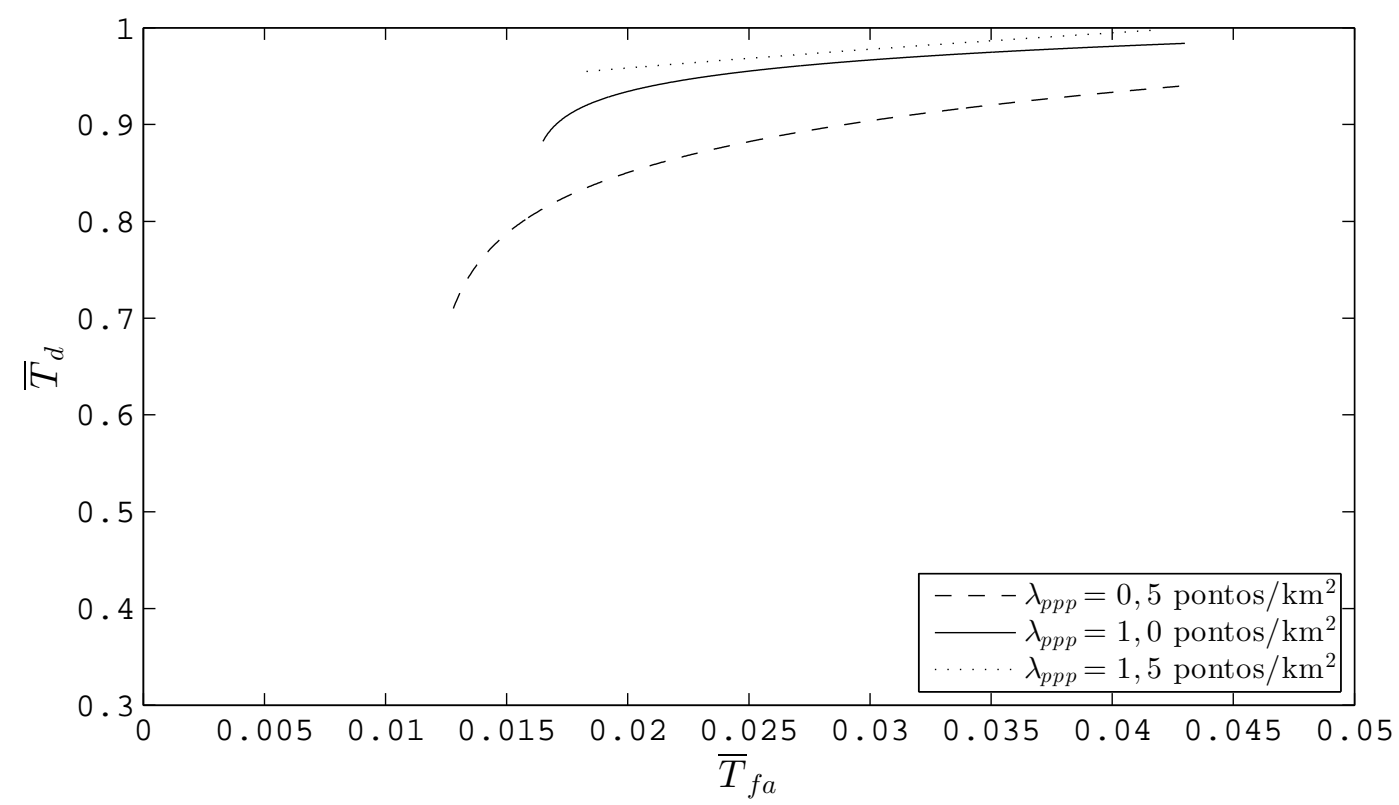

Figura 5.13: Desempenho dos métodos de geração de REM: efeito da densidade de USs, Cenário 1, Caso 1

próximo do desempenho do Método_1 com $\ell=0,55$.

Observa-se ainda que o pequeno aumento na Taxa Espacial de Detecção média proporcionado pelo Método_0 se faz ao custo de um aumento percentual maior na Taxa Espacial de Falso Alarme média, que se reflete numa redução de capacidade no HDFSS. Na Figura 5.12, por exemplo, observa-se que um aumento de aproximadamente $0,8 \%$ em $\bar{T}_{d}$ (de 0,971 para 0,979 ) é obtido às custas de um aumento de aproximadamente $12 \%$ em $\bar{T}_{f a}$ (de 0,031 para 0,035 ). 
Cenário 1, Caso 2: Usuário primário com DEP plana Neste caso, os valores dos pares $\left(T_{f a}, T_{d}\right)$ obtidos nos casos de $\lambda_{p p p}=0,5, \lambda_{p p p}=1,0$ e $\lambda_{p p p}=1,5$ pontos $/ \mathrm{km}^{2}$ (5000 valores para cada método analisado) são apresentados nas figuras 5.14, 5.15 e 5.16, respectivamente.

Nestas figuras são ainda mostrados, através de asteriscos, os pontos médios $\left(\bar{T}_{f a}, \bar{T}_{d}\right)$ associados ao método Método_1, além da curva do tipo ROC $\left(\bar{T}_{f a}\right.$ versus $\left.\bar{T}_{d}\right)$ que passa por estes pontos. Para efeito de comparação, o ponto médio associado ao Método_0 é também indicado na figura, através de um triangulo.

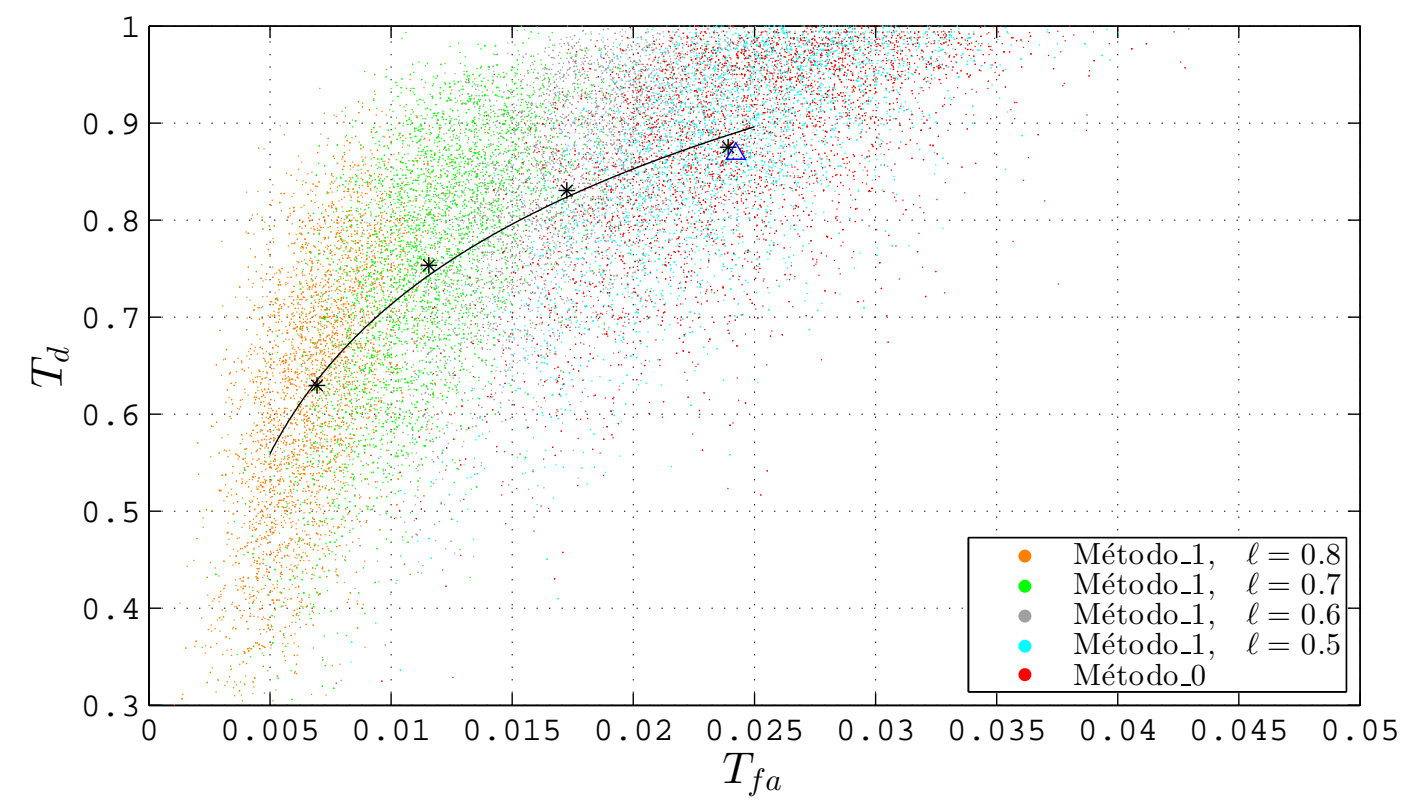

Figura 5.14: Pares $\left(T_{f a}, T_{d}\right)$ obtidos para o Cenário 1 , Caso 2 com $\lambda_{p p p}=$ 0,5 pontos $/ \mathrm{km}^{2}$

O efeito produzido por variações na densidade média de usuários secundários pode ser observada na Figura 5.17, onde são presentadas as curvas do $\bar{T}_{f a}$ versus $\bar{T}_{d}$ associadas a $\lambda_{p p p}=0,5, \lambda_{p p p}=1,0$ e $\lambda_{p p p}=1,5$ pontos $/ \mathrm{km}^{2}$.

As curvas na Figura 5.17 mostram que, conforme o número médio de usuários secundários presentes na região de interesse aumenta, o desempenho dos métodos de geração de REM melhora. Note que, por exemplo, para um valor de $\bar{T}_{f a}=0,15$ o Método_1 apresenta um aumento na $\bar{T}_{d}$ de 0,796 para 0,935 quando a densidade de pontos $\lambda_{\text {ppp }}$ aumenta de 0,5 para 1,5.

Com base nas figuras 5.14, 5.15 e 5.16 pode-se observar que, em ambos os métodos de geração de REM, a variância dos pontos $\left(T_{f a}, T_{d}\right)$, refletida no tamanho das nuvens de pontos obtidas, diminui conforme a densidade de usuários secundários na região de interesse aumenta. Isto acontece porque a precisão dos mapas REM gerados aumenta com o número de USs que 


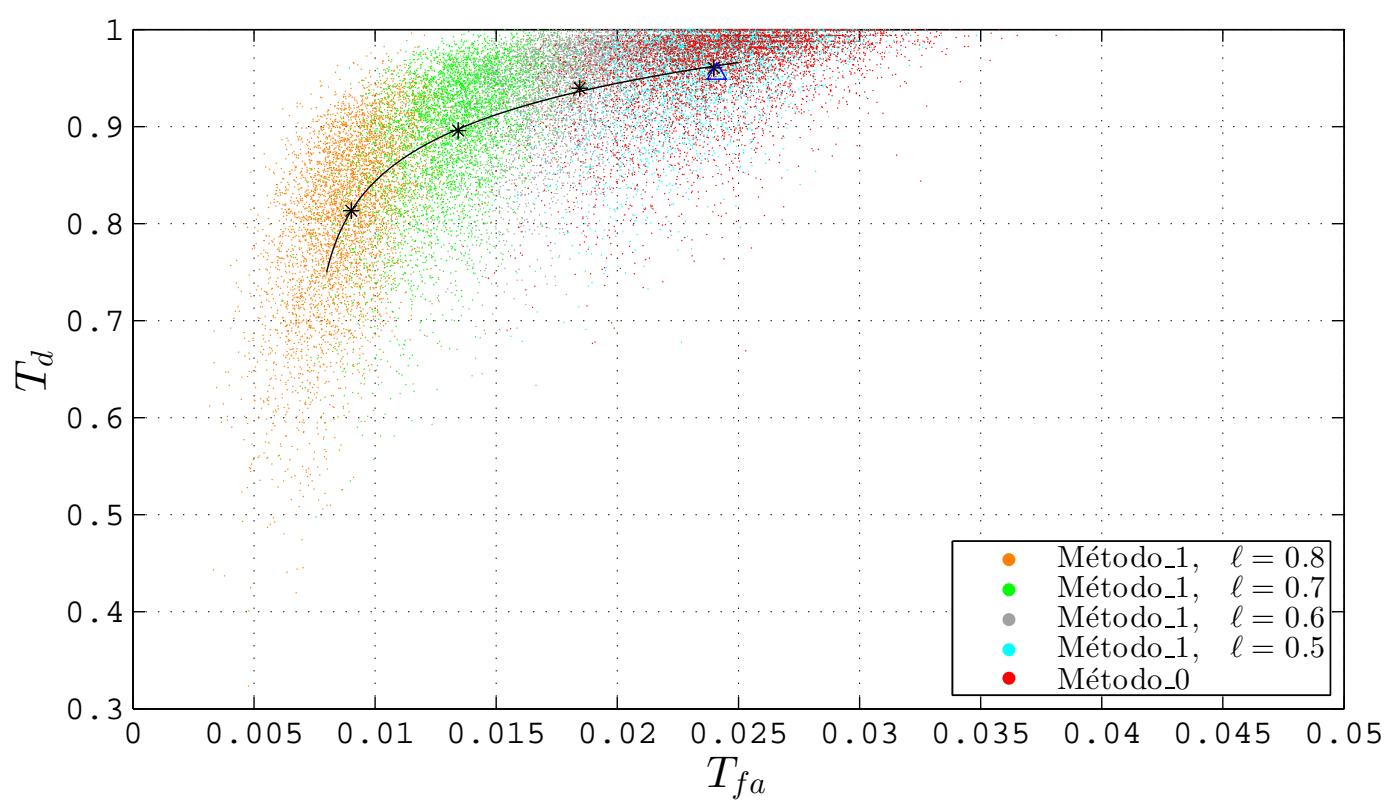

Figura 5.15: Pares $\left(T_{f a}, T_{d}\right)$ obtidos para o Cenário 1, Caso 2 com $\lambda_{p p p}=$ 1 pontos $/ \mathrm{km}^{2}$

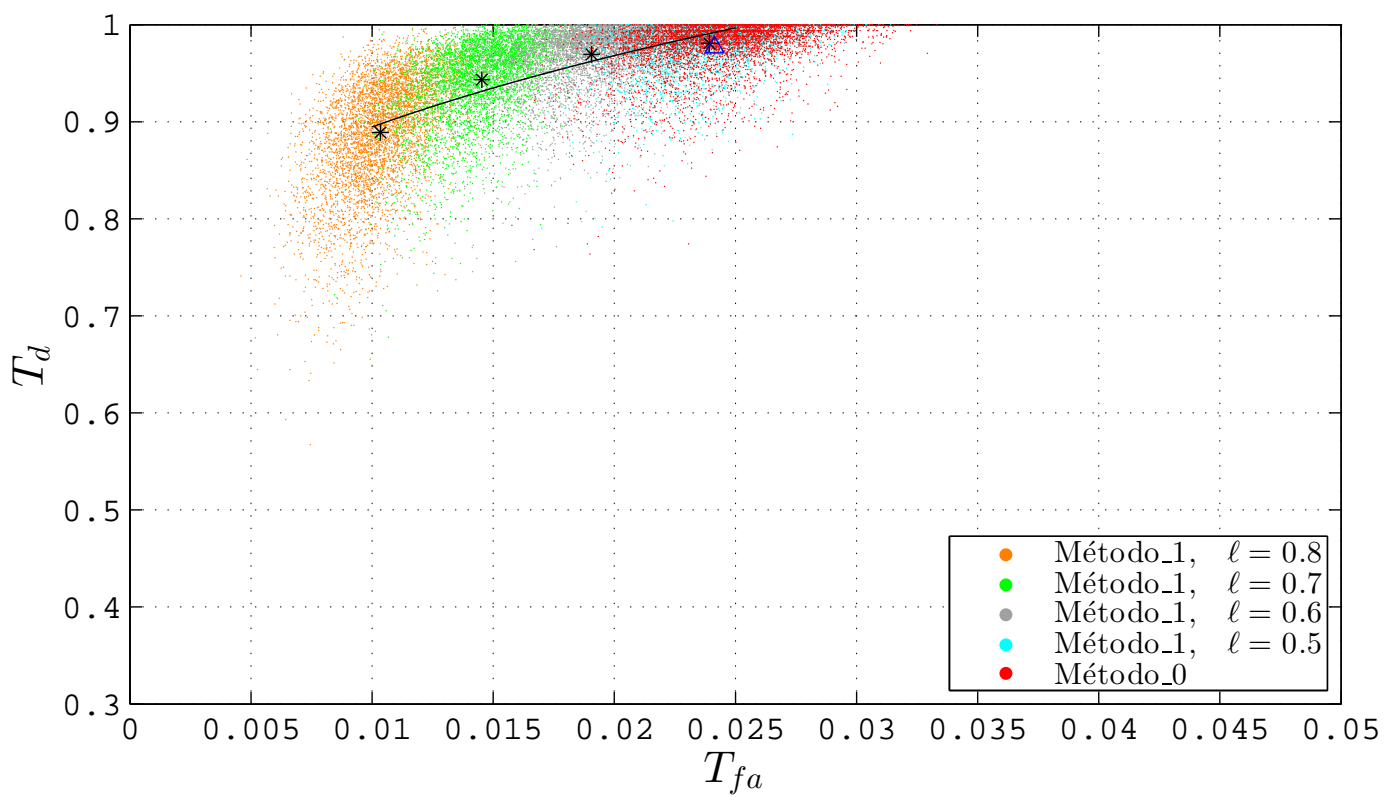

Figura 5.16: Pares $\left(T_{f a}, T_{d}\right)$ obtidos para o Cenário 1, Caso 2 com $\lambda_{p p p}=$ 1,5 pontos $/ \mathrm{km}^{2}$

estejam cooperando. Note que a partir destas curvas é possível determinar, conhecido o valor da densidade de pontos $\lambda_{p p p}$, o valor do nível de quantização $\ell$ mais adequado para o ponto de operação desejado $\left(\bar{T}_{f a}, \bar{T}_{d}\right)$. Um outro ponto observado é que, em todos os casos, o desempenho do Método_0 é bastante próximo do desempenho do Método_ 1 com $\ell=0,5$.

Observa-se ainda que o pequeno aumento na Taxa Espacial de Detecção 


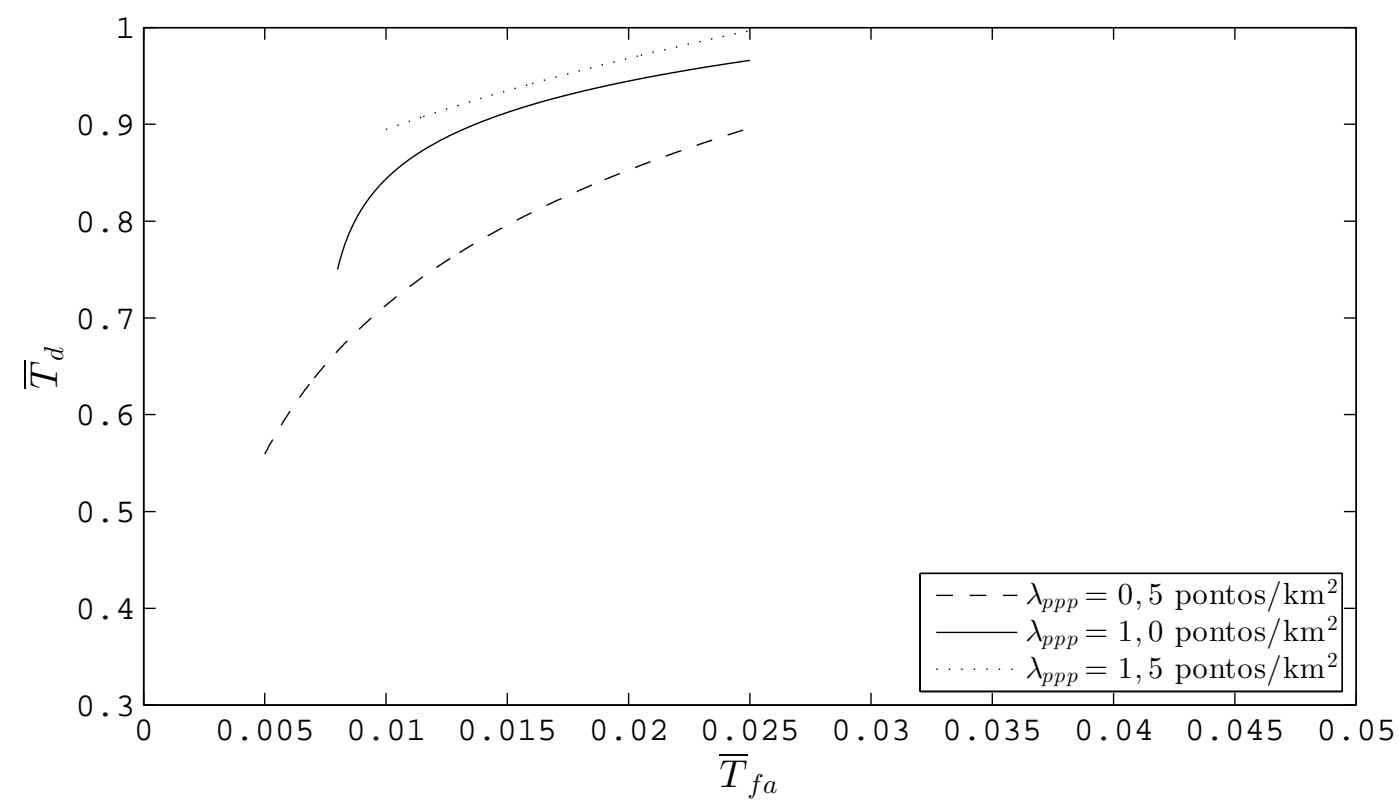

Figura 5.17: Desempenho dos métodos de geração de REM: efeito da densidade de USs, Cenário 1, Caso 2

média proporcionado pelo Método__ se faz ao custo de um aumento percentual maior na Taxa Espacial de Falso Alarme média, que se reflete numa redução de capacidade no HDFSS. Na Figura 5.15, por exemplo, observa-se que um aumento de aproximadamente $1,5 \%$ em $\bar{T}_{d}$ (de 0,939 para 0,954 ) é obtido às custas de um aumento de aproximadamente 30,7\% em $\bar{T}_{f a}$ (de 0,0184 para 0,0241).

Os resultados obtidos no Caso 2 indicam que quando o sinal do usuário primário tem DEP plana (amostras descorrelatadas), é possível se trabalhar com Taxas Espaciais de Falso Alarme menores do que as taxas mínimas obtidas quando os sinais dos UPs têm DEP de dois polos (Caso 1). Note que esta redução em $\bar{T}_{f a}$ é às custas de uma redução não desprezível em $\bar{T}_{d}$.

Os resultados correspondentes ao Cenário 1, Caso 2, deram origem a um artigo publicado nos anais da Computing and Communication Workshop and Conference - CCWC de 2018 [27]. 


\section{Cenário 1, Caso 3: usuário primário com DEP de 2 polos mas considerado} pela DBC como sendo plana Os valores dos pares $\left(T_{f a}, T_{d}\right)$ obtidos nos casos de $\lambda_{p p p}=0,5, \lambda_{p p p}=1,0$ e $\lambda_{p p p}=1,5$ pontos $/ \mathrm{km}^{2}$ (5000 valores para cada método analisado) são apresentados nas figuras 5.18, 5.19 e 5.20, respectivamente.

Nestas figuras são ainda mostrados, através de asteriscos, os pontos médios $\left(\bar{T}_{f a}, \bar{T}_{d}\right)$ associados ao método Método_1, além da curva do tipo ROC $\left(\bar{T}_{f a}\right.$ versus $\left.\bar{T}_{d}\right)$ que passa por estes pontos. Para efeito de comparação, o ponto médio associado ao Método_0 é também indicado na figura, através de um triangulo.

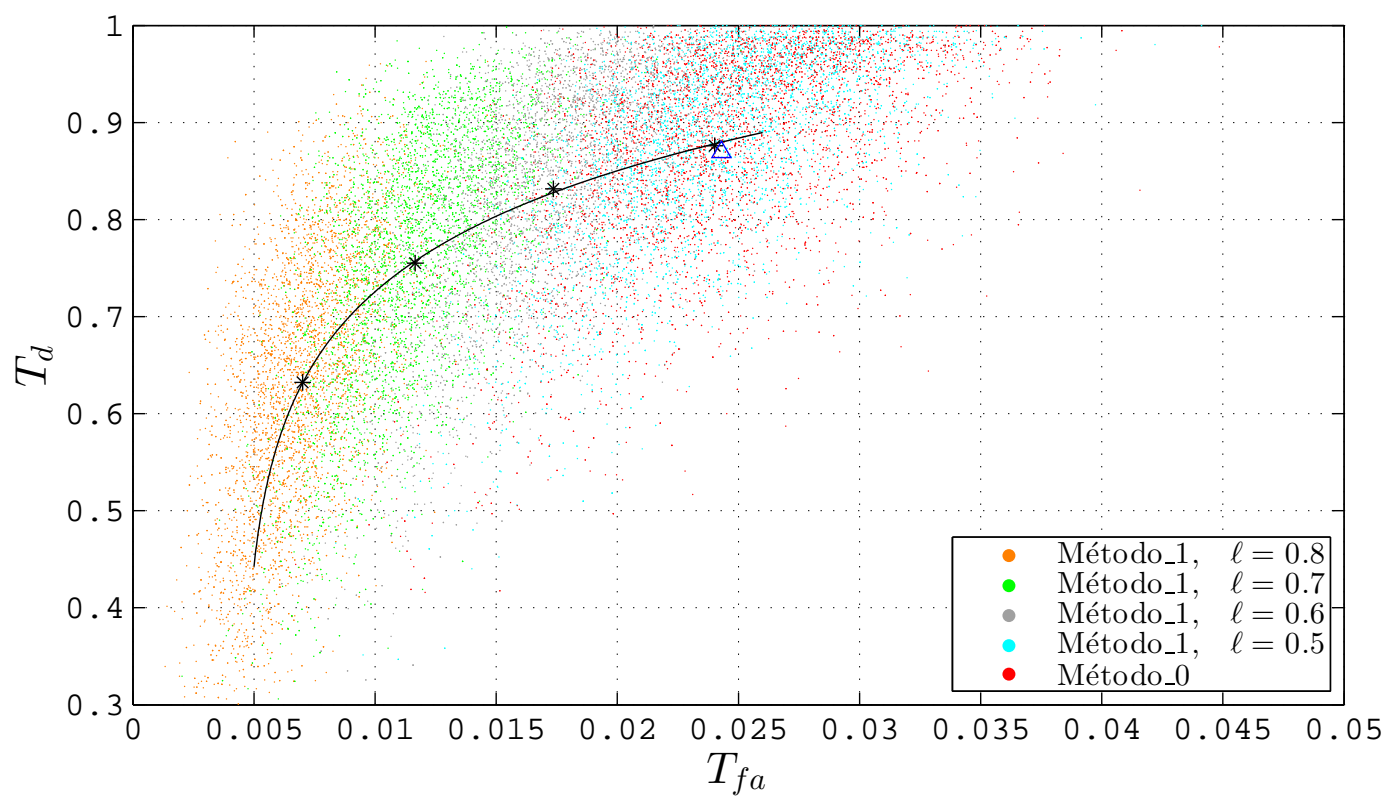

Figura 5.18: Pares $\left(T_{f a}, T_{d}\right)$ obtidos para o Cenário 1, Caso 3 com $\lambda_{p p p}=$ 0,5 pontos $/ \mathrm{km}^{2}$

Neste caso, o efeito produzido por variações na densidade média de usuários secundários pode ser observada na Figura 5.21, onde são presentadas as curvas em vermelho do $\bar{T}_{f a}$ versus $\bar{T}_{d}$ associadas a $\lambda_{p p p}=0,5, \lambda_{p p p}=1,0 \mathrm{e}$ $\lambda_{\text {ppp }}=1,5$ pontos $/ \mathrm{km}^{2}$.

Como foi definido no início da Seção 5.4, no terceiro caso é avaliada uma situação onde, embora o sinal produzido pelas transmissões dos usuários primários tenha uma densidade espectral de potência de dois polos, o Centro de Base de Dados gera o Mapa de Ambiente de Rádio considerando que a densidade espectral de potência dos sinais transmitidos pelos usuários primários é plana.

Neste caso, embora as curvas da Figura 5.21 indiquem que para valores de $\bar{T}_{f a}$ no intervalo $[0,017,0,025]$ os valores de $\bar{T}_{d}$ correspondentes às situações 


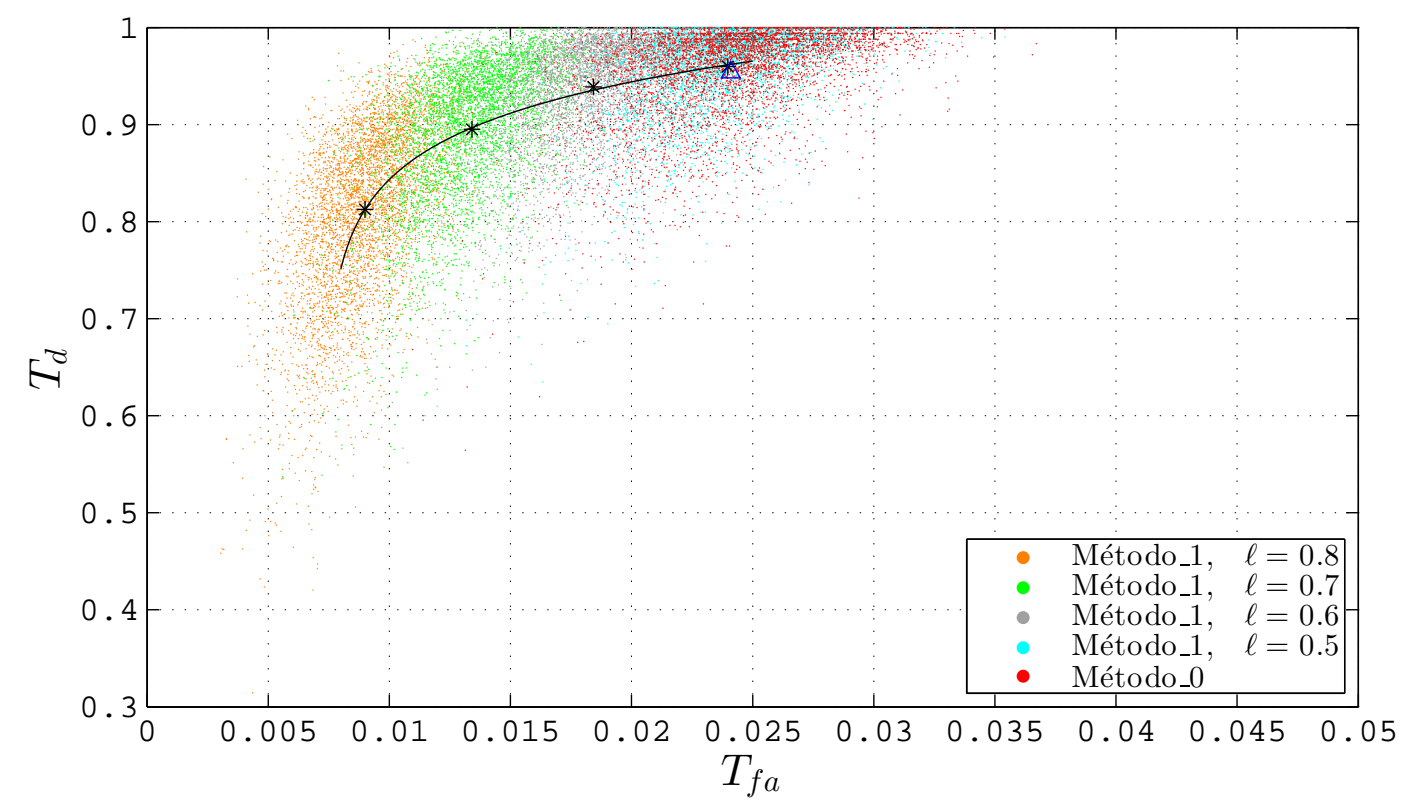

Figura 5.19: Pares $\left(T_{f a}, T_{d}\right)$ obtidos para o Cenário 1, Caso $3 \mathrm{com} \lambda_{p p p}=$ 1 pontos $/ \mathrm{km}^{2}$

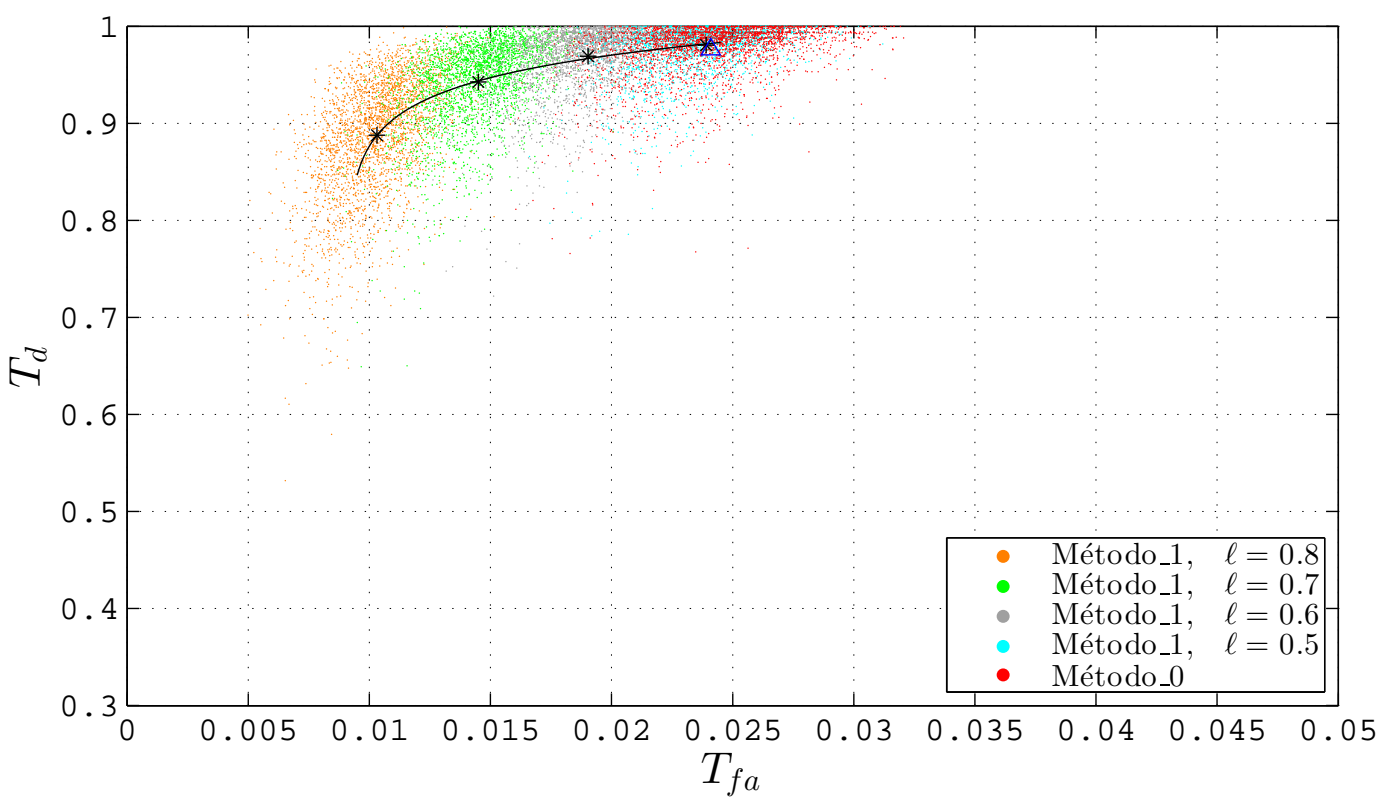

Figura 5.20: Pares $\left(T_{f a}, T_{d}\right)$ obtidos para o Cenário 1, Caso $3 \mathrm{com} \lambda_{p p p}=$ 1,5 pontos $/ \mathrm{km}^{2}$

analisadas nos casos 1 e 3 sejam bastante próximos, uma comparação das figuras 5.10, 5.11 e 5.12 respectivamente com as figuras 5.18, 5.19 e 5.20 mostra que no Caso 1, no qual o Centro de Base de Dados gera o REM considerando o formato real da DEP dos sinais dos UPs, a variância dos pontos $\left(T_{f a}, T_{d}\right)$, (refletida no tamanho das nuvens de pontos), é menor de que no Caso 3. 


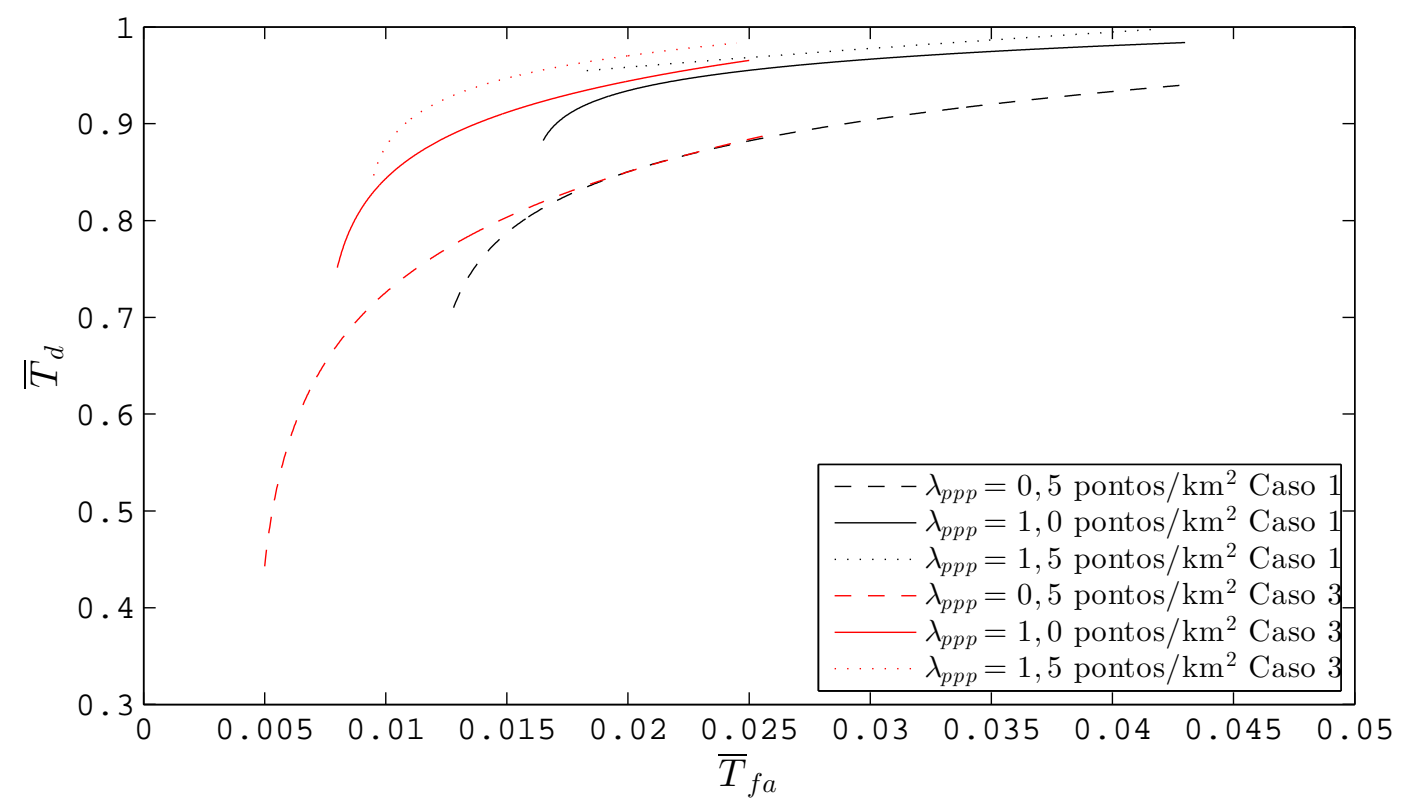

Figura 5.21: Desempenho dos métodos de geração de REM: comparação dos casos 1 e 3, Cenário 1 


\section{4 .2}

\section{Cenário 2: três usuários primários operando na região de interesse}

Neste cenário, as localizações dos transmissores e receptores dos três enlaces do Serviço Fixo Terrestre considerados são apresentadas na Tabela 5.2. O percurso de estes enlaces está ilustrado na Figura 5.22 onde o contorno em verde indica a fronteira da área de serviço do HDFSS.

Tabela 5.2: Coordenadas dos transmissores e receptores dos três enlace FS considerados.

\begin{tabular}{|c|c|c|c|c|}
\hline UP & Long do Tx & Lat do Tx & Lon do Rx & Lat do Rx \\
\hline 1 & $59,92^{\circ} \mathrm{O}$ & $0,1038^{\circ} \mathrm{N}$ & $60,16^{\circ} \mathrm{O}$ & $0,0568^{\circ} \mathrm{N}$ \\
\hline 2 & $59,89^{\circ} \mathrm{O}$ & $0,0020^{\circ} \mathrm{S}$ & $60,15^{\circ} \mathrm{O}$ & $0,06^{\circ} \mathrm{S}$ \\
\hline 3 & $59,88^{\circ} \mathrm{O}$ & $0,0920^{\circ} \mathrm{S}$ & $60,07^{\circ} \mathrm{O}$ & $0,129^{\circ} \mathrm{N}$ \\
\hline
\end{tabular}

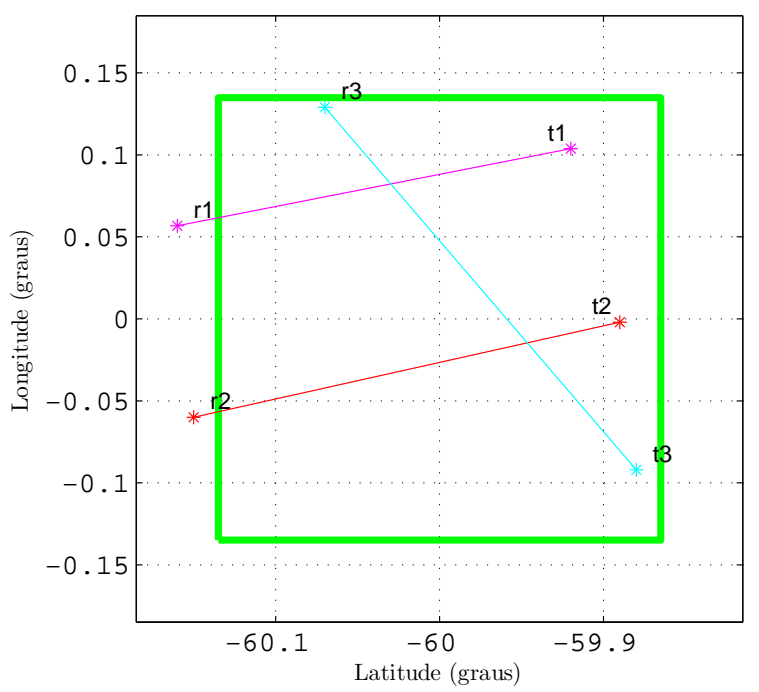

Figura 5.22: Localização dos três UPs na região de interesse

A partir dos parâmetros técnicos considerados para os enlaces FS e HDFSS nas seções 5.1 e 5.2 respectivamente, a densidade de potência interferente agregada produzida pelos transmissores FS na saída da antena receptora de um terminal HDFSS foi determinada para todas as possíveis posições do terminal HDFSS dentro de sua área de serviço $S_{T}$. O resultado obtido é apresentado na Figura 5.23.

Note que este resultado é importante para a determinação do Mapa de Ambiente de Rádio de referência $\left(S_{d_{r e f}}\right)$, definido como a região da área de serviço $S_{T}$ onde a densidade de potência interferente agregada produzida pelos transmissores FS na saída da antena de um terminal HDFSS é superior ou igual a um valor máximo tolerável $I_{\max }$ de interferência. A Figura 5.24 apresenta o 
REM de referência obtido a partir da Figura 5.23 considerando-se um nível de $I_{\max }=-139 \mathrm{~dB}(\mathrm{~W} / \mathrm{MHz})$. Este valor de $I_{\max }$ é sugerido pela Recomendação ITU-R F.758 [50] para utilização em estudos de compartilhamento envolvendo o FS e outros serviços.

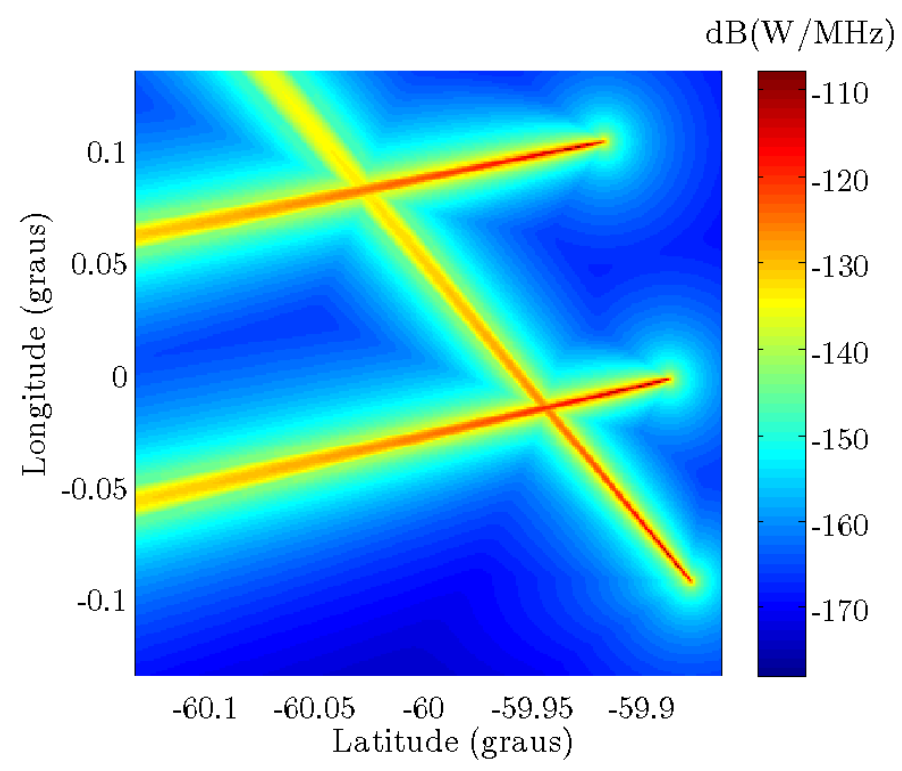

Figura 5.23: Densidade de potência interferente agregada produzida pelos transmissores FS na saída da antena receptora do terminal HDFSS em função da sua localização.

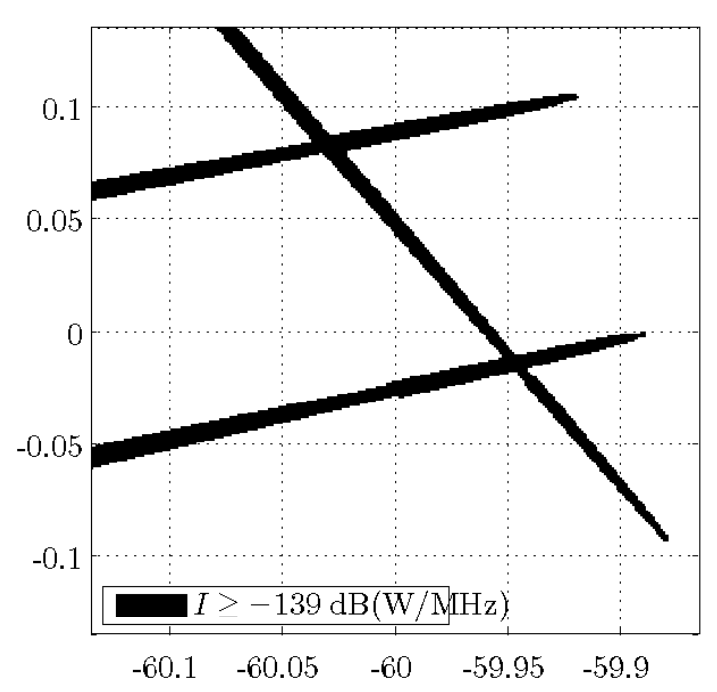

Figura 5.24: Mapa de Ambiente de Rádio de referência $\left(S_{d_{r e f}}\right)$ para um nível de $I_{\max }=-139 \mathrm{~dB}(\mathrm{~W} / \mathrm{MHz})$.

Considerando-se este segundo cenário, os resultados relativos aos três casos identificados no início da Seção 5.4 são apresentados a seguir. 
Cenário 2, Caso 1: usuários primários com DEP de 2 polos Os valores dos pares $\left(T_{f a}, T_{d}\right)$ obtidos nos casos de $\lambda_{p p p}=0,5, \lambda_{p p p}=1,0$ e $\lambda_{p p p}=$ 1,5 pontos $/ \mathrm{km}^{2}$ (5000 valores para cada método analisado) são apresentados nas figuras 5.25, 5.26 e 5.27, respectivamente.

Nestas figuras são ainda mostrados, através de asteriscos, os pontos médios $\left(\bar{T}_{f a}, \bar{T}_{d}\right)$ associados ao método Método_1, além da curva do tipo ROC $\left(\bar{T}_{f a}\right.$ versus $\left.\bar{T}_{d}\right)$ que passa por estes pontos. Para efeito de comparação, o ponto médio associado ao Método_0 é também indicado na figura, através de um triangulo. Note que estas curvas são importantes para a escolha do nível de quantização $\ell$ a ser utilizado no Método_1 de geração de REM, permitindo a escolha do nível de quantização mais adequado.

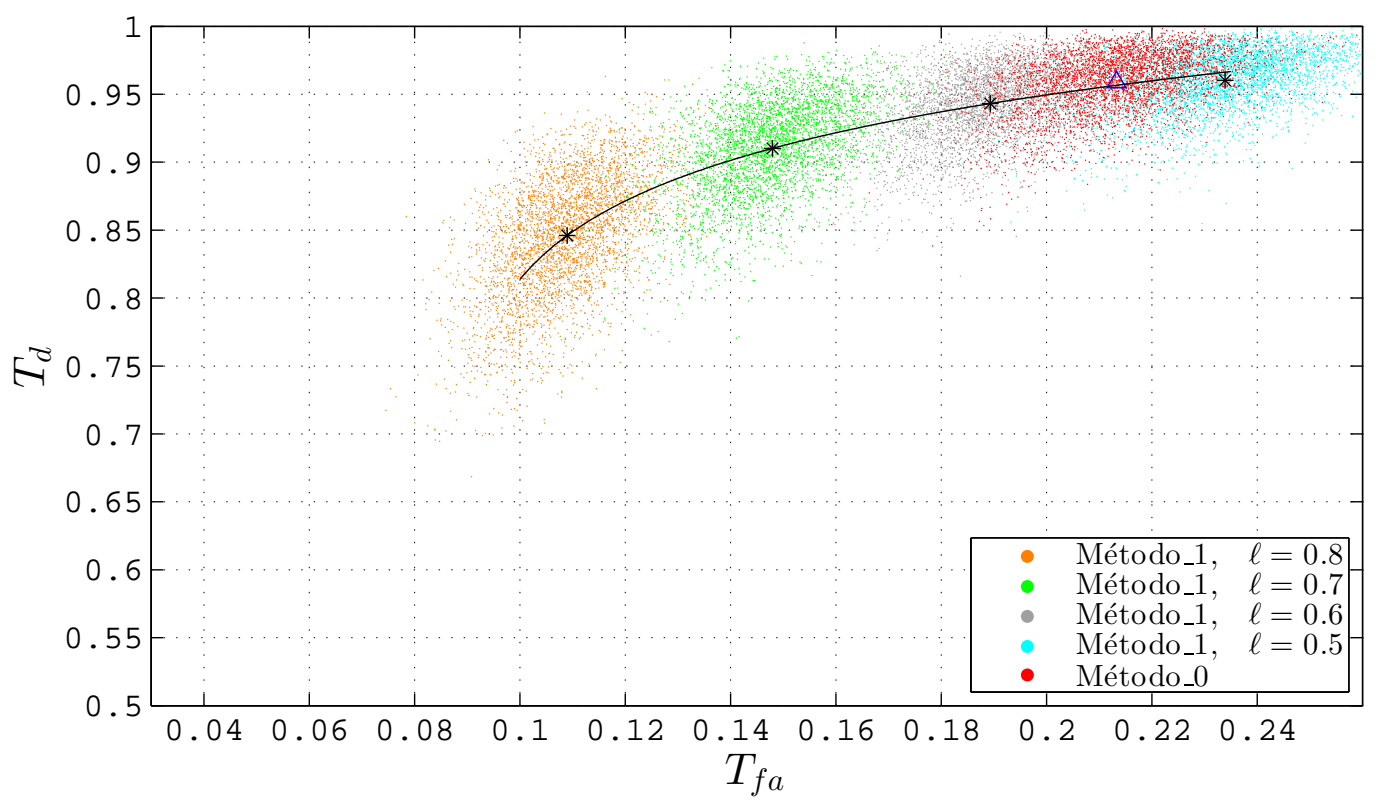

Figura 5.25: Pares $\left(T_{f a}, T_{d}\right)$ obtidos para o Cenário 2, Caso 1 com $\lambda_{p p p}=$ 0,5 pontos $/ \mathrm{km}^{2}$

Uma avaliação comparativa de desempenho dos métodos de geração de REM quando aplicados a diferentes densidades de USs pode ser apreciada na Figura 5.28.

As curvas na Figura 5.28 mostram que, conforme o número médio de usuários secundários presentes na região de interesse aumenta, o desempenho dos métodos de geração de REM melhora. Note que, por exemplo, para um valor de $\bar{T}_{f a}=0,14$ o Método_1 apresenta um aumento na $\bar{T}_{d}$ de 0,901 para 0,977 quando a densidade de pontos $\lambda_{\text {ppp }}$ aumenta de 0,5 para 1,5.

Com base nas figuras 5.25, 5.26 e 5.27 pode-se observar que, em ambos os métodos de geração de REM, a variância dos pontos $\left(T_{f a}, T_{d}\right)$, refletida no tamanho das nuvens de pontos obtidas, diminui conforme a densidade de 


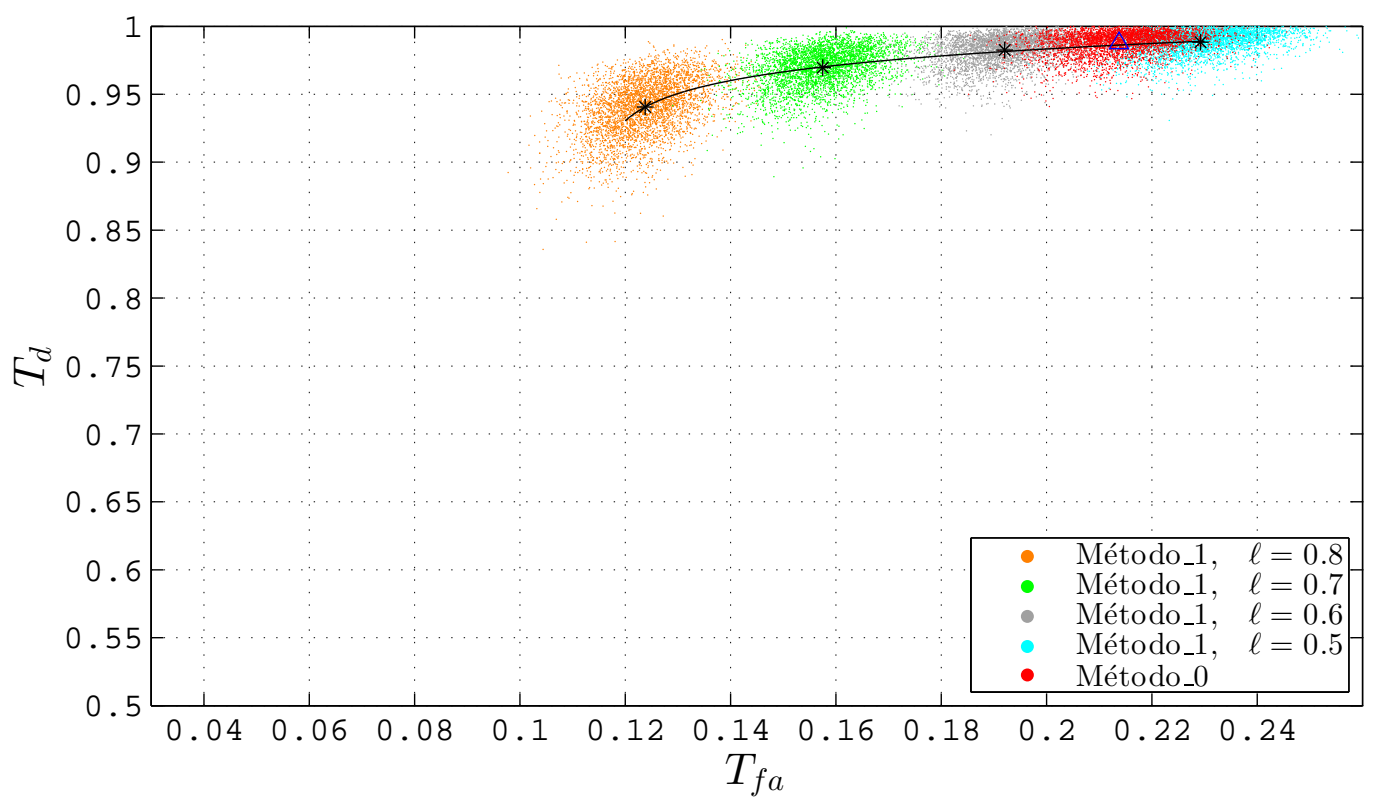

Figura 5.26: Pares $\left(T_{f a}, T_{d}\right)$ obtidos para o Cenário 2, Caso 1 com $\lambda_{p p p}=$ 1 pontos $/ \mathrm{km}^{2}$

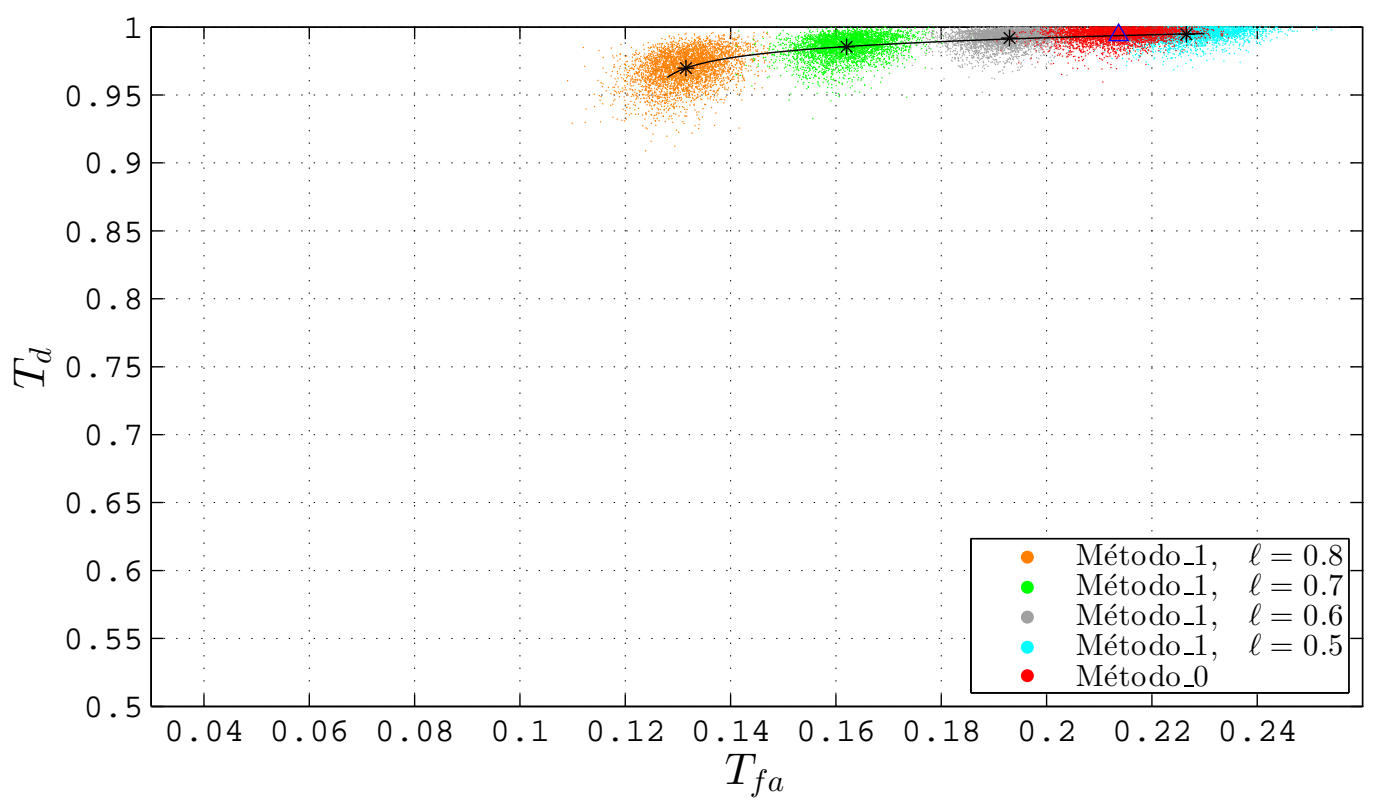

Figura 5.27: Pares $\left(T_{f a}, T_{d}\right)$ obtidos para o Cenário 2, Caso 1 com $\lambda_{p p p}=$ 1,5 pontos $/ \mathrm{km}^{2}$

usuários secundários na região de interesse aumenta. Isto acontece porque a precisão dos mapas REM gerados aumenta com o número de USs que estejam cooperando. Note que a partir destas curvas é possível determinar, conhecido o valor da densidade de pontos $\lambda_{p p p}$, o valor do nível de quantização $\ell$ mais adequado para o ponto de operação desejado $\left(\bar{T}_{f a}, \bar{T}_{d}\right)$. Um outro ponto 


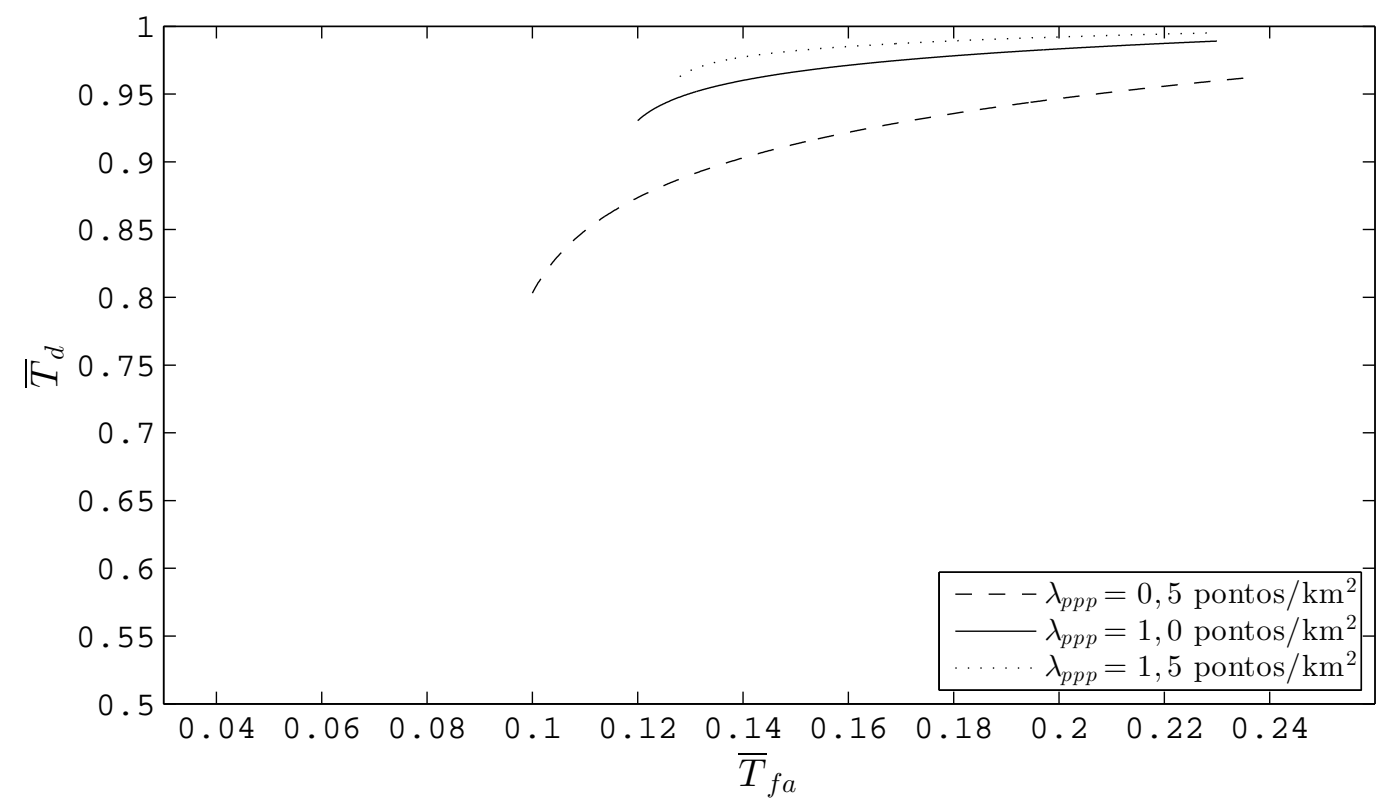

Figura 5.28: Desempenho dos métodos de geração de REM: efeito da densidade de USs, Cenário 2, Caso 1

observado é que, em todos os casos, o desempenho do Método_0 é bastante próximo do desempenho do Método_1 com $\ell=0,55$.

Observa-se ainda que o pequeno aumento na Taxa Espacial de Detecção média proporcionado pelo Método_0 se faz ao custo de um aumento percentual maior na Taxa Espacial de Falso Alarme média, que se reflete numa redução de capacidade no HDFSS. Na Figura 5.25, por exemplo, observa-se que um aumento de aproximadamente $1,7 \%$ em $\bar{T}_{d}$ (de 0,943 para 0,958 ) é obtido às custas de um aumento de aproximadamente $11,2 \%$ em $\bar{T}_{f a}$ (de 0,1893 para $0,2133)$. 
Cenário 2, Caso 2: usuários primários com DEP plana Neste caso, os valores dos pares $\left(T_{f a}, T_{d}\right)$ obtidos nos casos de $\lambda_{p p p}=0,5, \lambda_{p p p}=1,0$ e $\lambda_{p p p}=1,5$ pontos $/ \mathrm{km}^{2}$ (5000 valores para cada método analisado) são apresentados nas figuras 5.29, 5.30 e 5.31, respectivamente.

Nestas figuras são ainda mostrados, através de asteriscos, os pontos médios $\left(\bar{T}_{f a}, \bar{T}_{d}\right)$ associados ao método Método_1, além da curva do tipo ROC $\left(\bar{T}_{f a}\right.$ versus $\left.\bar{T}_{d}\right)$ que passa por estes pontos. Para efeito de comparação, o ponto médio associado ao Método_0 é também indicado na figura, através de um triangulo.

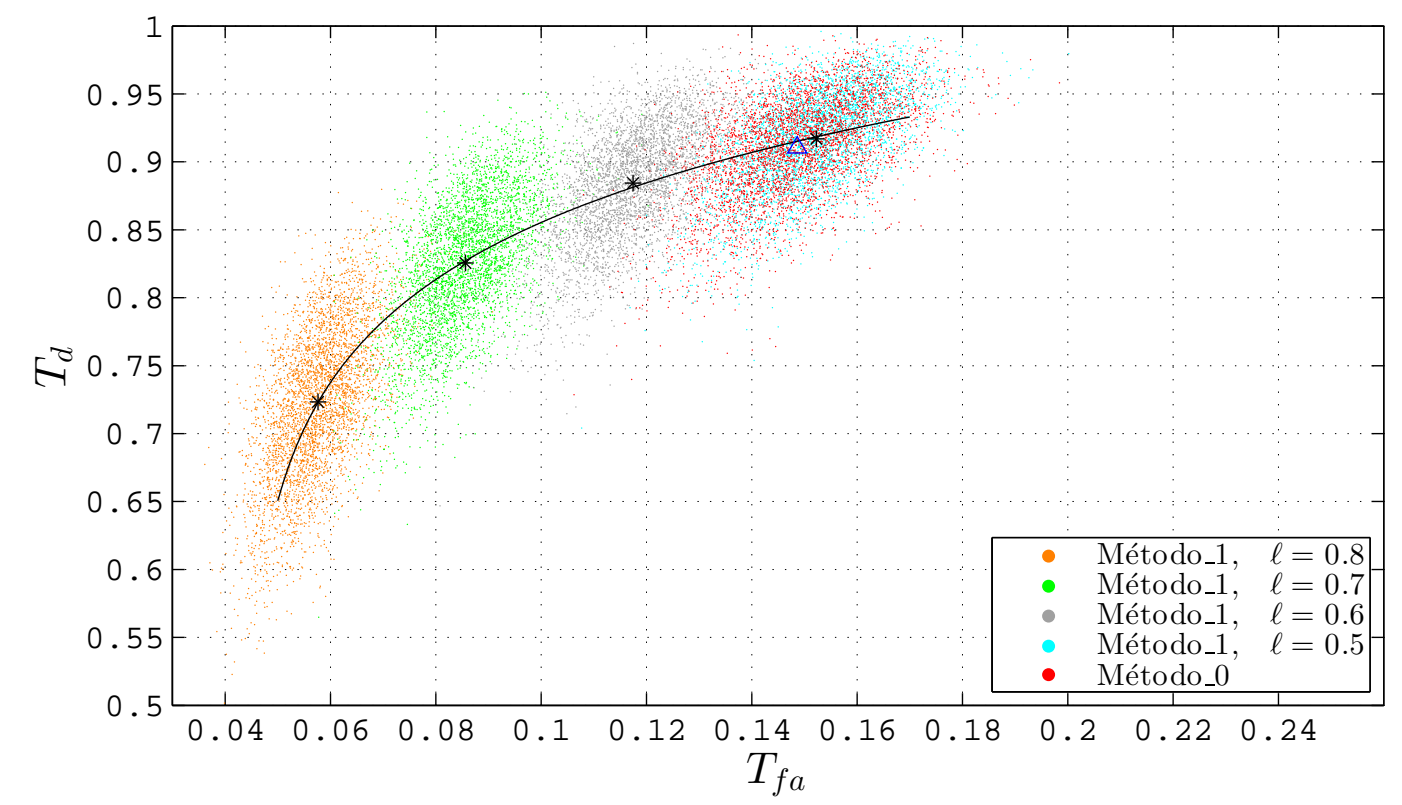

Figura 5.29: Pares $\left(T_{f a}, T_{d}\right)$ obtidos para o Cenário 2, Caso 2 com $\lambda_{p p p}=$ 0,5 pontos $/ \mathrm{km}^{2}$

O efeito produzido por variações na densidade média de usuários secundários pode ser observada na Figura 5.32, onde são presentadas as curvas do $\bar{T}_{f a}$ versus $\bar{T}_{d}$ associadas a $\lambda_{p p p}=0,5, \lambda_{p p p}=1,0$ e $\lambda_{p p p}=1,5$ pontos $/ \mathrm{km}^{2}$.

As curvas na Figura 5.32 mostram que, conforme o número médio de usuários secundários presentes na região de interesse aumenta, o desempenho dos métodos de geração de REM melhora. Note que, por exemplo, para um valor de $\bar{T}_{f a}=0,1$ o Método_1 apresenta um aumento na $\bar{T}_{d}$ de 0,855 para 0,964 quando a densidade de pontos $\lambda_{\text {ppp }}$ aumenta de 0,5 para 1,5.

Com base nas figuras 5.29, 5.30 e 5.31 pode-se observar que, em ambos os métodos de geração de REM, a variância dos pontos $\left(T_{f a}, T_{d}\right)$, refletida no tamanho das nuvens de pontos obtidas, diminui conforme a densidade de usuários secundários na região de interesse aumenta. Isto acontece porque a precisão dos mapas REM gerados aumenta com o número de USs que 


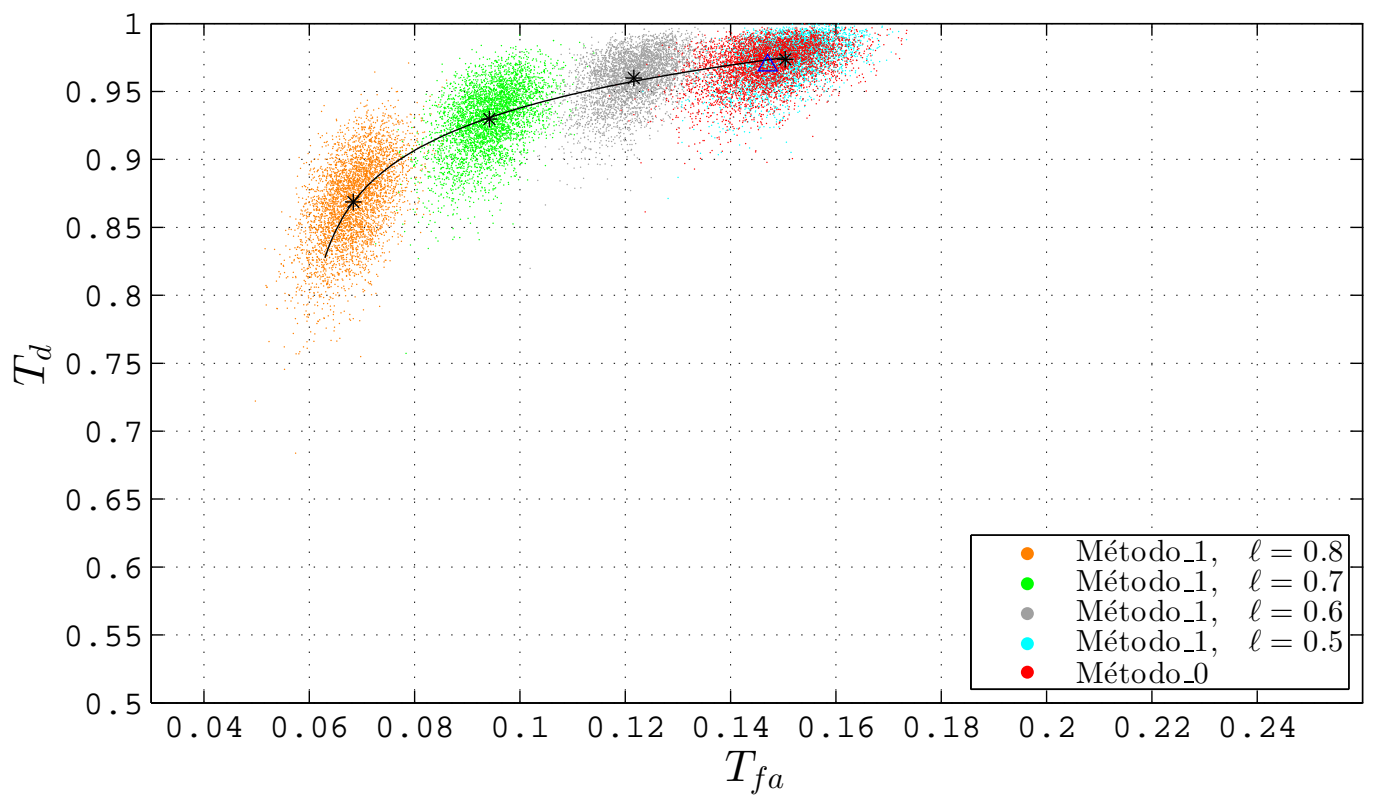

Figura 5.30: Pares $\left(T_{f a}, T_{d}\right)$ obtidos para o Cenário 2, Caso 2 com $\lambda_{p p p}=$ 1 pontos $/ \mathrm{km}^{2}$

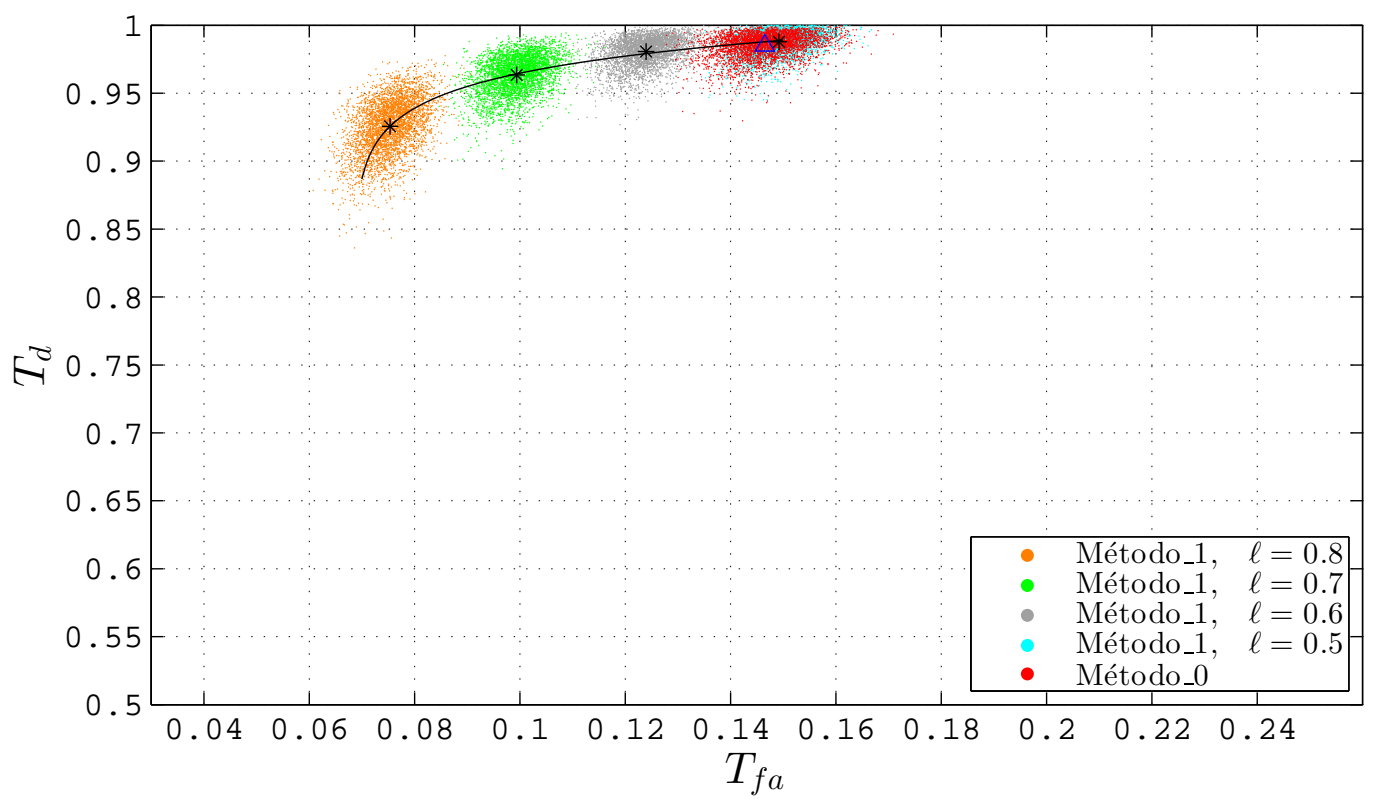

Figura 5.31: Pares $\left(T_{f a}, T_{d}\right)$ obtidos para o Cenário 2, Caso 2 com $\lambda_{p p p}=$ 1,5 pontos $/ \mathrm{km}^{2}$

estejam cooperando. Note que a partir destas curvas é possível determinar, conhecido o valor da densidade de pontos $\lambda_{p p p}$, o valor do nível de quantização $\ell$ mais adequado para o ponto de operação desejado $\left(\bar{T}_{f a}, \bar{T}_{d}\right)$. Um outro ponto observado é que, em todos os casos, o desempenho do Método_0 é bastante próximo do desempenho do Método_1 com $\ell=0,5$.

Observa-se ainda que o pequeno aumento na Taxa Espacial de Detecção 


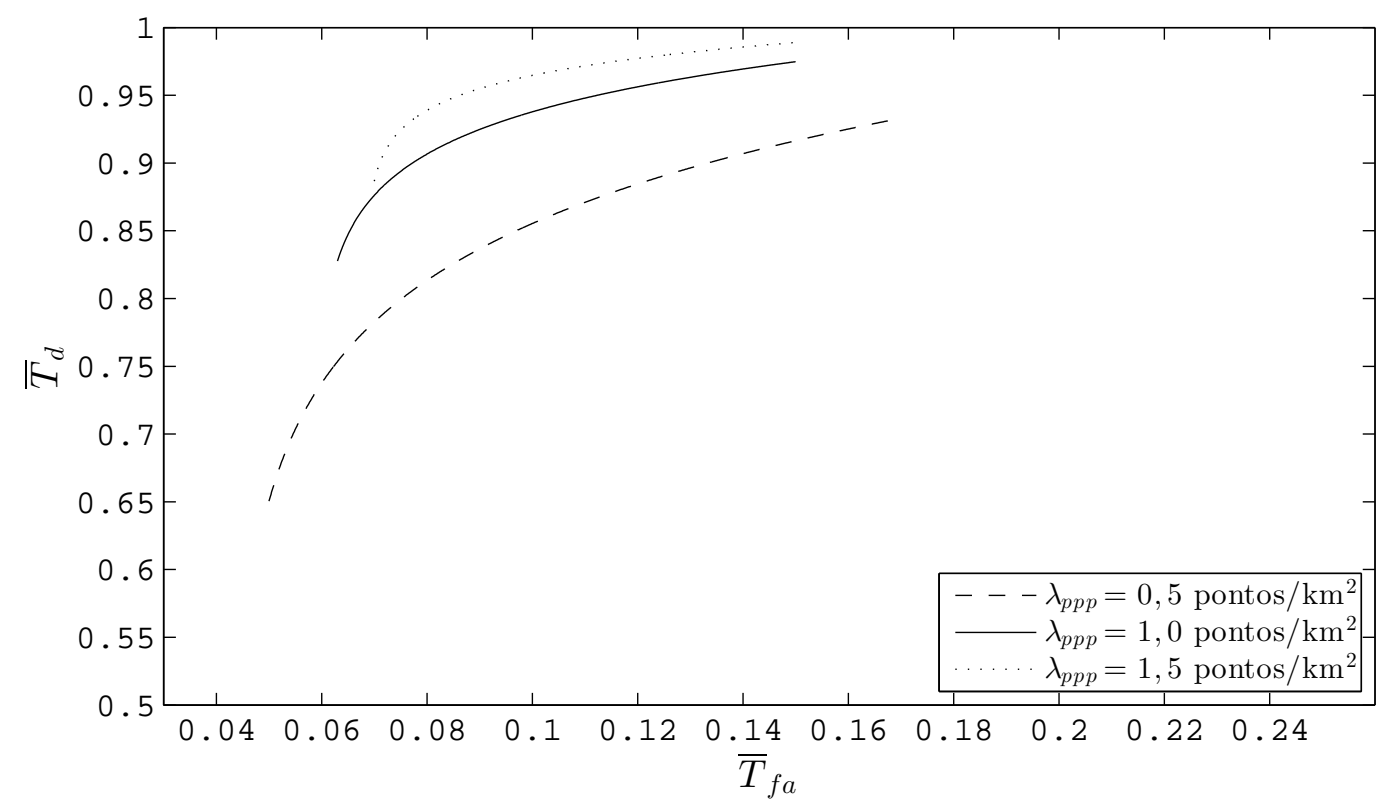

Figura 5.32: Desempenho dos métodos de geração de REM: efeito da densidade de USs, Cenário 2, Caso 2

média proporcionado pelo Método__ se faz ao custo de um aumento percentual maior na Taxa Espacial de Falso Alarme média, que se reflete numa redução de capacidade no HDFSS. Na Figura 5.29, por exemplo, observa-se que um aumento de aproximadamente $2,96 \% \mathrm{em} \bar{T}_{d}$ (de 0,884 para 0,910$)$ é obtido às custas de um aumento de aproximadamente $26,46 \%$ em $\bar{T}_{f a}$ (de 0,1175 para 0,1486).

Os resultados obtidos no Caso 2 indicam que quando o sinal do usuário primário tem DEP plana (amostras descorrelatadas), é possível se trabalhar com Taxas Espaciais de Falso Alarme menores do que as taxas mínimas obtidas quando os sinais dos UPs têm DEP de dois polos (Caso 1). Note que esta redução em $\bar{T}_{f a}$ é às custas de uma redução não desprezível em $\bar{T}_{d}$.

Os resultados correspondentes ao Cenário2, Caso 2, deram origem a um artigo publicado nos anais do Simpósio Brasileiro de Telecomunicações e Processamento de Sinais de 2018 [33]. 


\section{Cenário 2, Caso 3: usuários primários com DEP de 2 polos mas conside-} rado pela BDC como sendo plana Os valores dos pares $\left(T_{f a}, T_{d}\right)$ obtidos nos casos de $\lambda_{p p p}=0,5, \lambda_{p p p}=1,0$ e $\lambda_{p p p}=1,5$ pontos $/ \mathrm{km}^{2}$ (5000 valores para cada método analisado) são apresentados nas figuras 5.33, 5.34 e 5.35, respectivamente. Nestas figuras são ainda mostrados, através de asteriscos, os pontos médios $\left(\bar{T}_{f a}, \bar{T}_{d}\right)$ associados ao método Método_1, além da curva do tipo ROC $\left(\bar{T}_{f a}\right.$ versus $\left.\bar{T}_{d}\right)$ que passa por estes pontos. Para efeito de comparação, o ponto médio associado ao Método_0 é também indicado na figura, através de um triangulo.

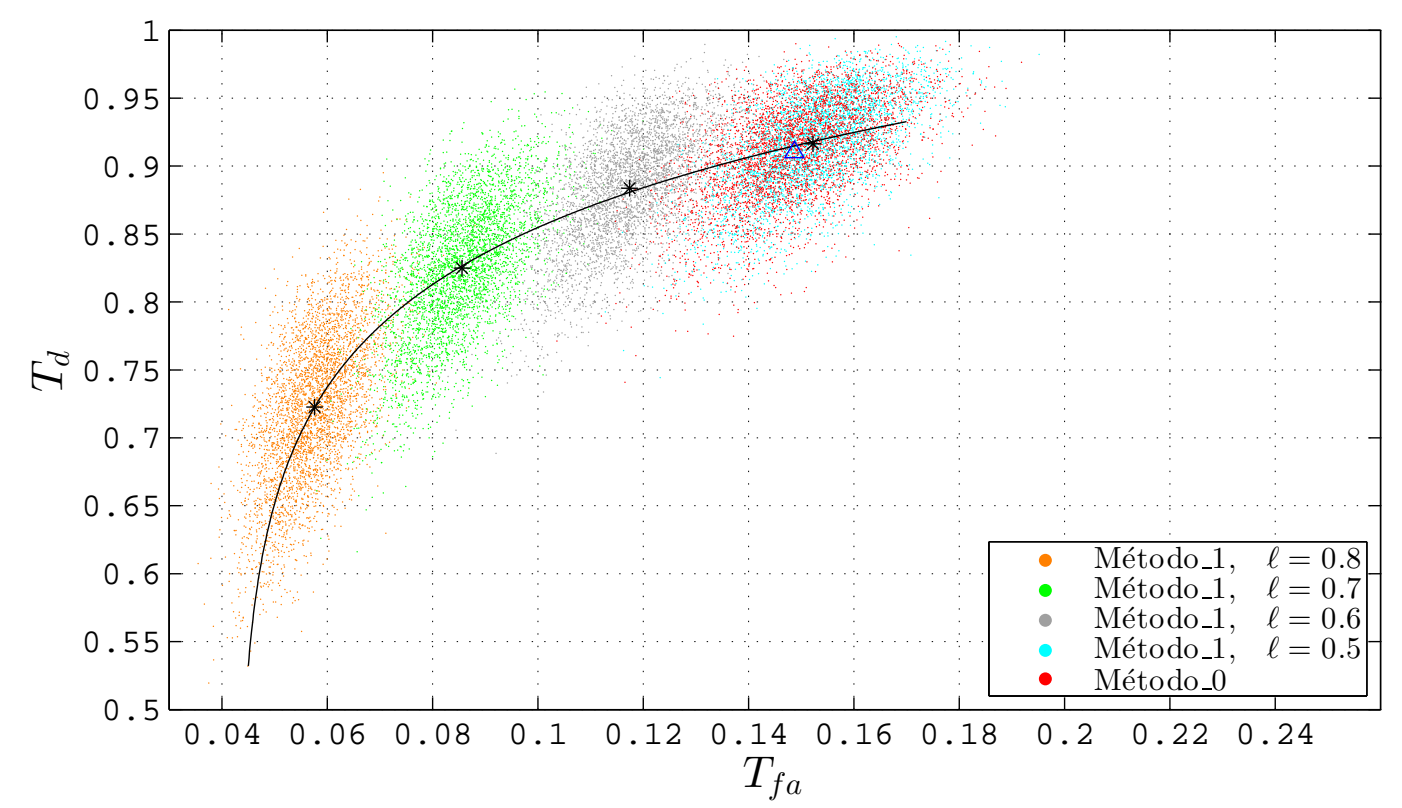

Figura 5.33: Pares $\left(T_{f a}, T_{d}\right)$ obtidos para o Cenário 2, Caso 3 com $\lambda_{p p p}=$ 0,5 pontos $/ \mathrm{km}^{2}$

Neste caso, o efeito produzido por variações na densidade média de usuários secundários pode ser observada na Figura 5.36, onde são presentadas as curvas do $\bar{T}_{f a}$ versus $\bar{T}_{d}$ associadas a $\lambda_{p p p}=0,5, \lambda_{p p p}=1,0$ e $\lambda_{p p p}=$ 1,5 pontos $/ \mathrm{km}^{2}$.

Como foi definido no início da Seção 5.4, no terceiro caso é avaliada uma situação onde, embora o sinal produzido pelas transmissões dos usuários primários tenha uma densidade espectral de potência de dois polos, o Centro de Base de Dados gera o Mapa de Ambiente de Rádio considerando que a densidade espectral de potência dos sinais transmitidos pelos usuários primários é plana.

Neste caso, embora as curvas da Figura 5.36 indiquem que para valores de $\bar{T}_{f a}$ no intervalo $[0,11,0,16]$ os valores de $\bar{T}_{d}$ correspondentes às situações analisadas nos casos 1 e 3 sejam bastante próximos, uma comparação das 


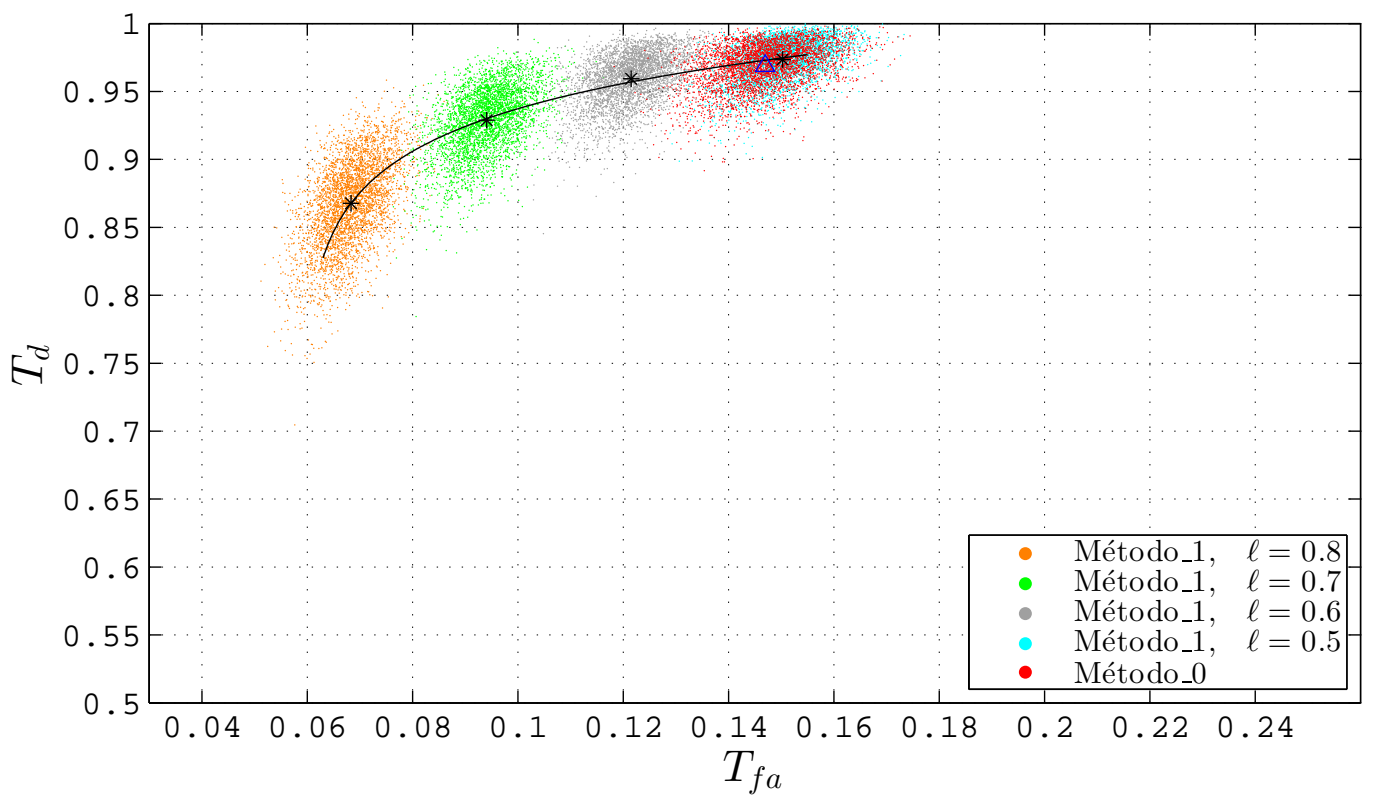

Figura 5.34: Pares $\left(T_{f a}, T_{d}\right)$ obtidos para o Cenário 2, Caso 3 com $\lambda_{p p p}=$ 1 pontos $/ \mathrm{km}^{2}$

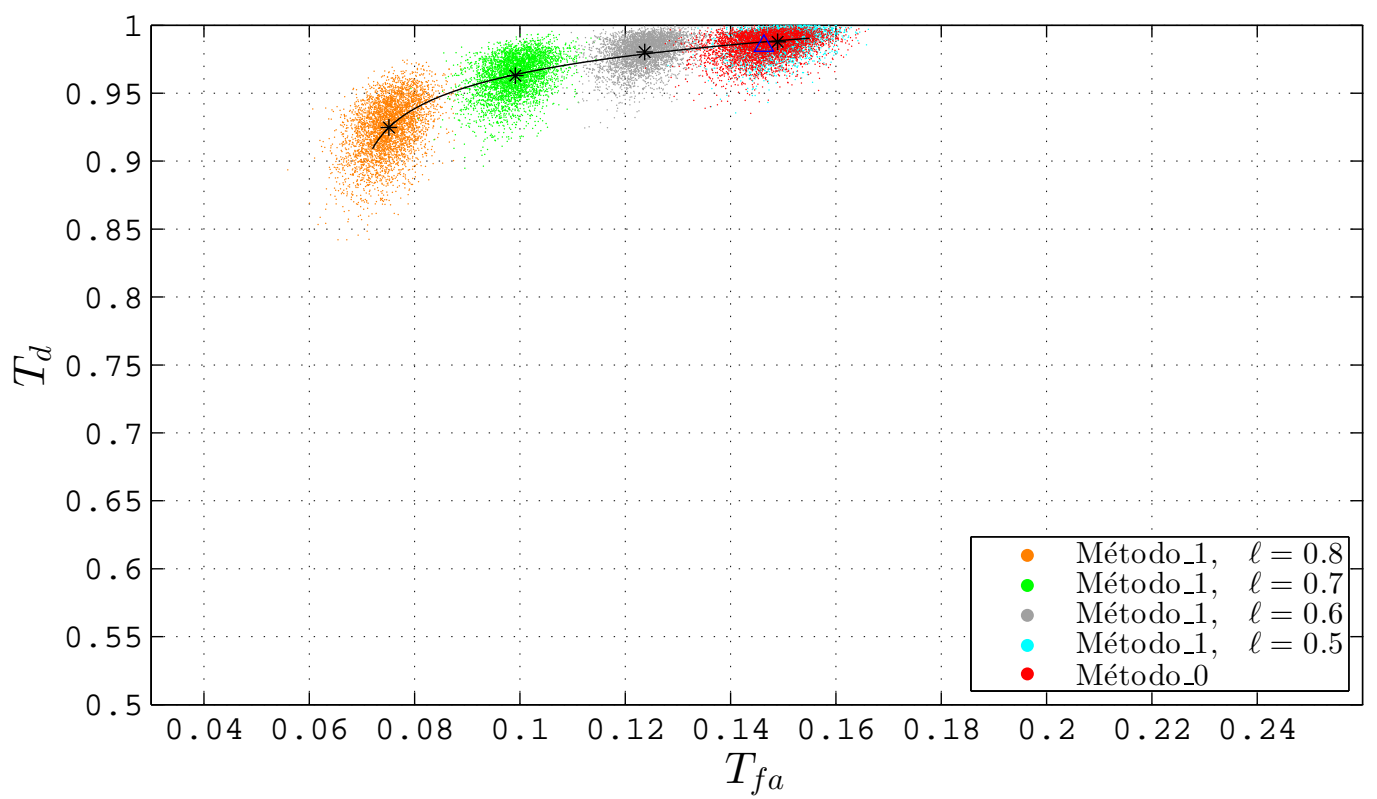

Figura 5.35: Pares $\left(T_{f a}, T_{d}\right)$ obtidos para o Cenário 2, Caso 3 com $\lambda_{p p p}=$ 1,5 pontos $/ \mathrm{km}^{2}$

figuras 5.25, 5.26 e 5.27 respectivamente com as figuras 5.33, 5.34 e 5.35 mostra que no Caso 1, no qual o Centro de Dados gera o REM considerando o formato real da DEP dos sinais dos UPs, a variância dos pontos $\left(T_{f a}, T_{d}\right)$, (refletida no tamanho das nuvens de pontos), é menor de que no Caso 3. 


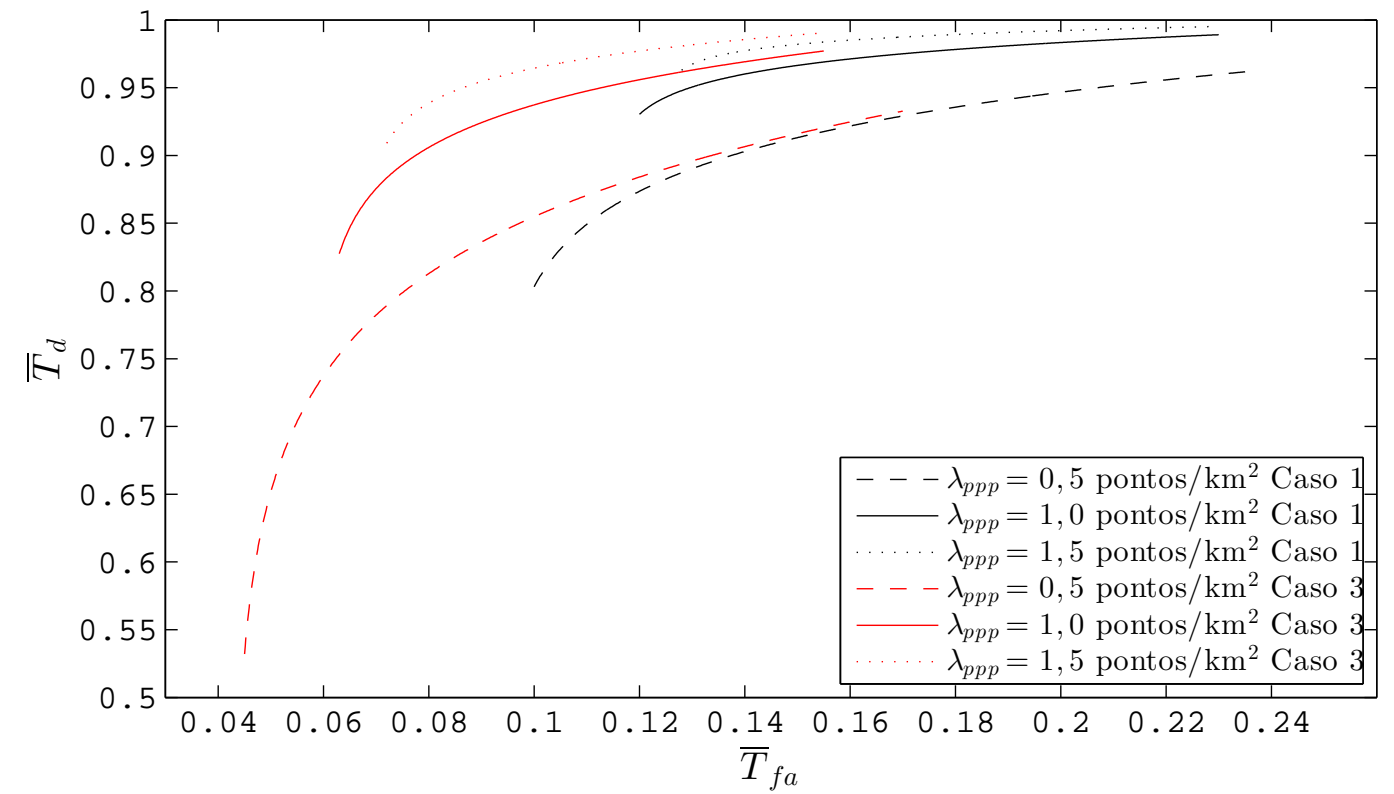

Figura 5.36: Desempenho dos métodos de geração de REM: comparação dos casos 1 e 3, Cenário 2 


\section{4 .3}

\section{Comentários adicionais relativos aos resultados obtidos nos cenários 1 e}

2

Nesta Subseção são apresentadas comparações entre os resultados obtidos para cada um dos dois cenários apresentados. As figuras 5.37, 5.38 e 5.39 apresentam conjuntos de curvas de desempenho dos métodos de geração de REM, obtidas para os cenários 1 e 2 e correspondentes aos casos 1, 2 e 3, respectivamente.

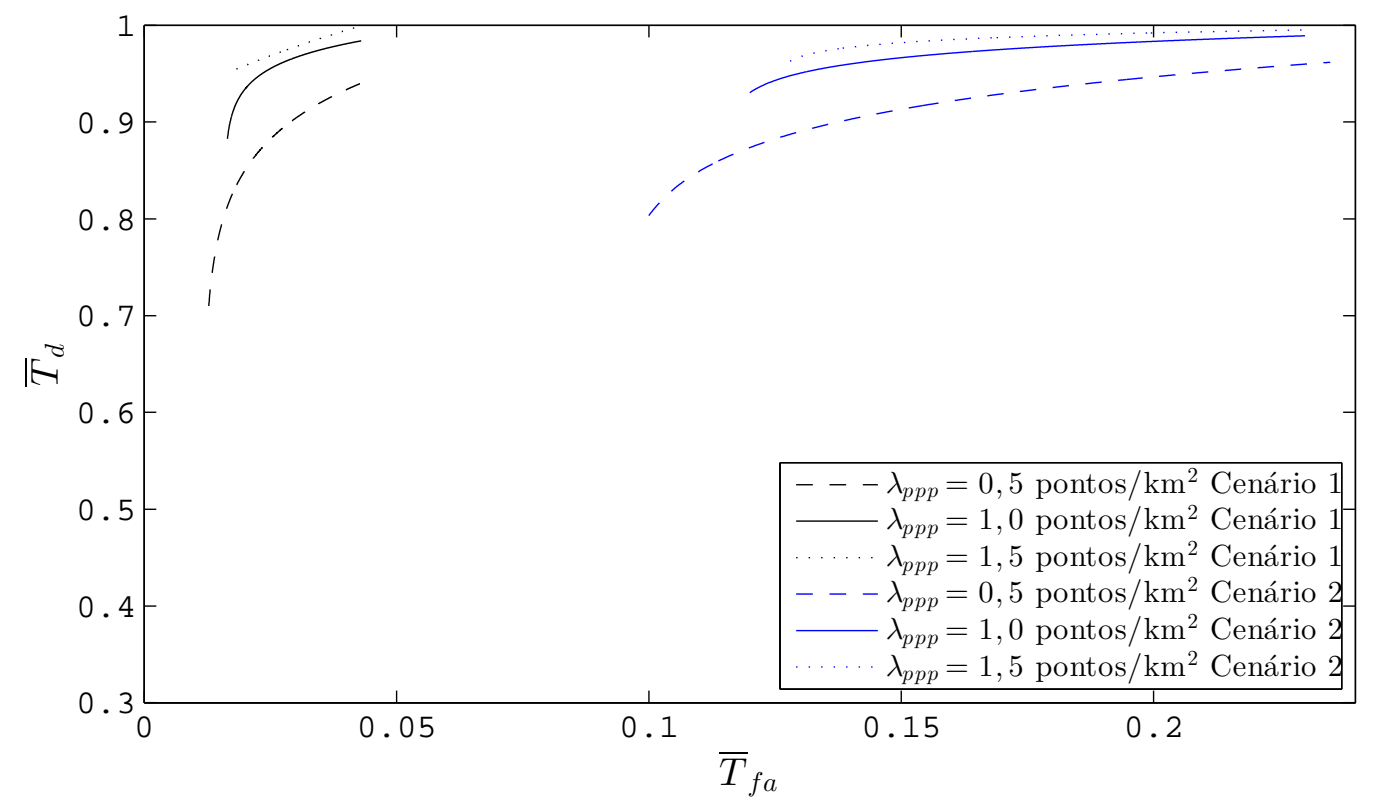

Figura 5.37: Desempenho dos métodos de geração de REM: comparação dos cenários 1 e 2, Caso 1

Note que os resultados apresentados nas figuras 5.37, 5.38 e 5.39 evidenciam que, embora os valores de $\bar{T}_{d}$ nos dois cenários estejam bem próximos, existe um aumento nos valores de $\bar{T}_{f a}$ do Cenário 2 em relação àqueles do Cenário 1. Isto sugere que a capacidade utilizada pelo sistema HDFSS diminui com o aumento do número de usuários primários operando na região de interesse. Observa-se ainda, que esta perda de capacidade é maior no Caso 1 do que no Caso 2 (ver figuras 5.37 e 5.38). 


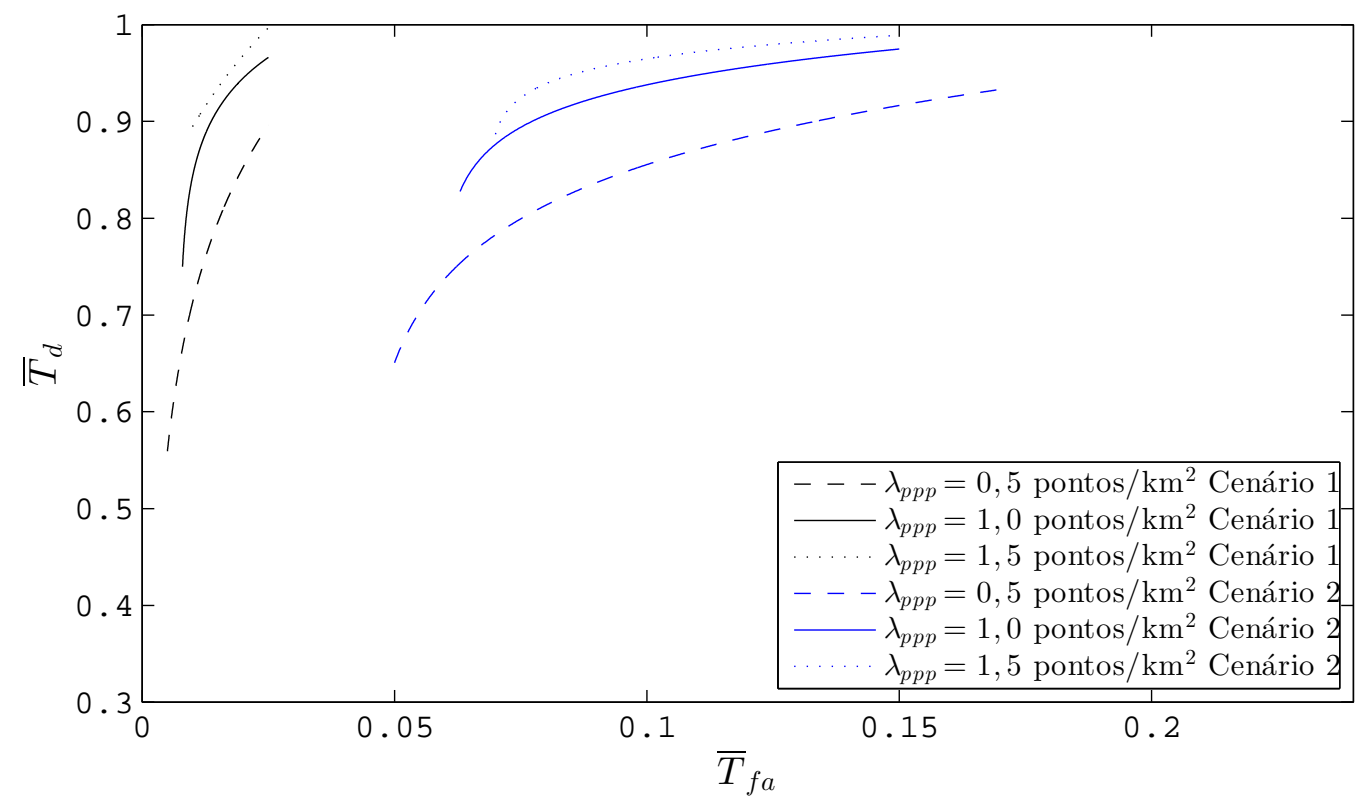

Figura 5.38: Desempenho dos métodos de geração de REM: comparação dos cenários 1 e 2, Caso 2

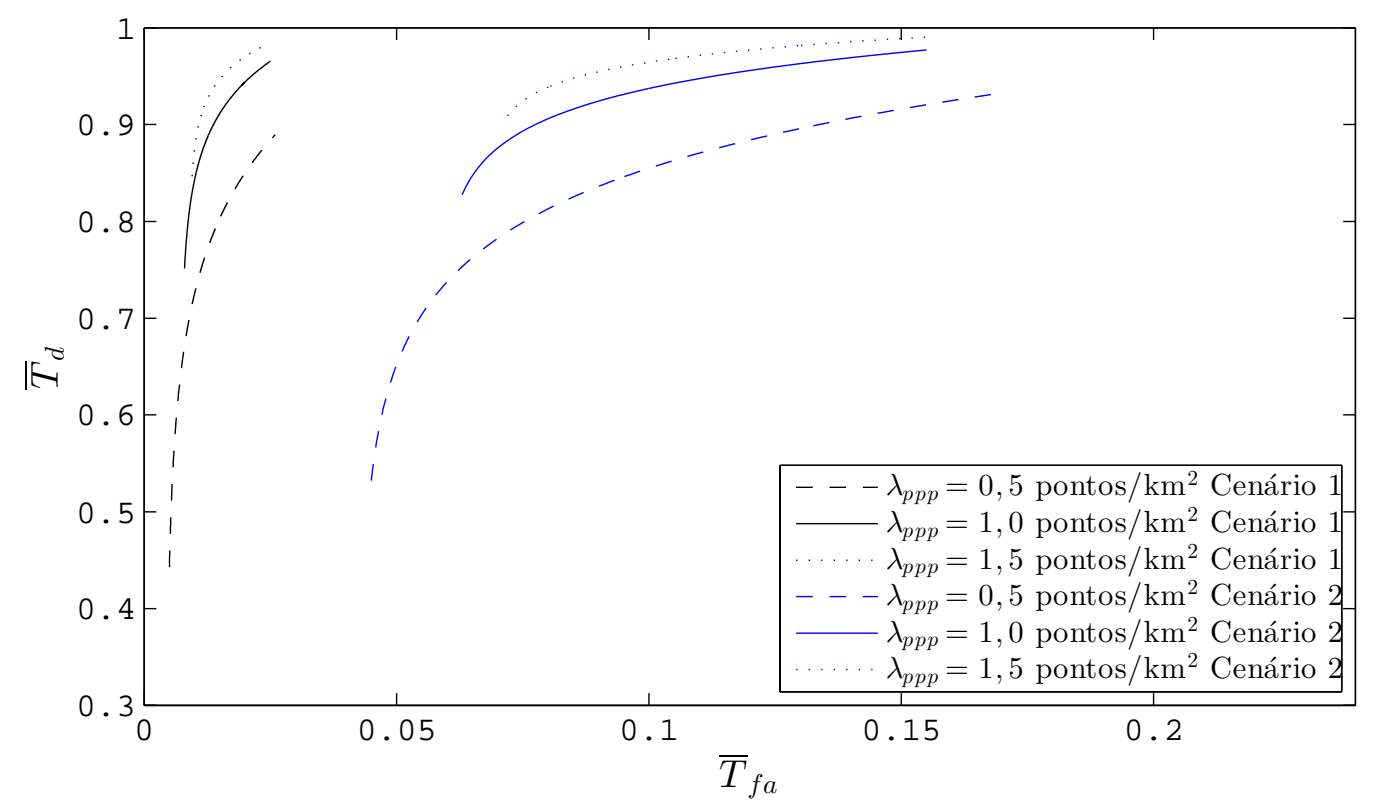

Figura 5.39: Desempenho dos métodos de geração de REM: comparação dos cenários 1 e 2, Caso 3 


\section{Conclusões}

Neste trabalho foi analisado um problema específico envolvendo o compartilhamento de frequências entre o Serviço Fixo Terrestre (FS) e uma aplicação de alta densidade do Serviço Fixo por Satélite (HDFSS). No cenário considerado, sistemas do FS (operando como usuários primários) e sistemas do HDFSS (operando como usuários secundários e utilizando rádios cognitivos) compartilham a mesma faixa de frequências em uma mesma região geográfica.

Para facilitar a convivência entre os dois serviços, o uso de um Mapa de Ambiente de Rádio (REM - Radio Environment Map) foi considerado. Um novo método de geração de REM foi proposto e avaliado. No desenvolvimento do método foi utilizada uma modelagem original que considera uma densidade espectral de potência genérica para os sinais transmitidos pelos usuários primários. Além disso, métricas adequadas para avaliar a qualidade de REMs e o desempenho dos métodos de geração foram definidas e propostas. Estas métricas foram utilizadas numa análise comparativa de desempenho envolvendo o método de geração de REM proposto e um método de geração existente. A análise foi feita com base nos resultados obtidos para cenários específicos que consideraram a existência de múltiplos enlaces FS na região de interesse.

Dois cenários foram analisados. O primeiro deles considerou apenas um enlace do FS na área de serviço do HDFSS e, no segundo, três enlaces do FS foram considerados. Em ambos os cenários a distribuição geográfica dos terminais HDFSS foi modelada por um Processo Pontual de Poisson bidimensional, homogêneo. Em cada um dos cenários, três diferentes casos foram analisados. No primeiro caso considerou-se que o sinal produzido pelas transmissões dos usuários primários é caracterizado por um processo estocástico passa-faixa gaussiano ESA, de média nula, com densidade espectral de potência de dois polos. No segundo caso considerou-se que o sinal produzido pelas transmissões dos usuários primários é caracterizado por um processo estocástico passa-faixa gaussiano ESA, de média nula, com densidade espectral de potência plana. Finalmente, num terceiro caso, analisou-se a situação onde, embora o sinal produzido pelas transmissões dos usuários primários tenha uma densidade espectral de potência de dois polos, o Centro de Base de Dados considerou, em seus cálculos, que a densidade espectral de potência dos sinais transmitidos 
pelos usuários primários é plana. Neste terceiro caso, foi possível analisar as possíveis variações, nos desempenhos dos métodos de geração de REM analisados, proporcionada pelo conhecimento da densidade espectral de potência dos sinais dos usuários primários.

A partir dos resultados obtidos nos cenários analisados, foi possível observar, em geral, os seguintes comportamentos:

- Conforme esperado, na medida em que o número médio de usuários secundários cooperativos presentes na região de interesse aumenta, a variância dos pontos $\left(T_{f a}, T_{d}\right)$, refletida no tamanho das nuvens de pontos obtidas, diminui, indicando uma melhora no desempenho dos métodos de geração de REM;

- As curvas do tipo ROC obtidas ( $\operatorname{curvas} \bar{T}_{f a}$ versus $\bar{T}_{d}$ ) permitem determinar, conhecido o valor da densidade de usuários secundários, o valor do nível de quantização $\ell$ mais adequado para o ponto de operação do método de geração de REM proposto;

- Quando o nível de quantização $\ell$ é igual a 0,5, o desempenho do método de geração de REM proposto, para o Caso 2 nos dois cenários analisados, é bem próximo do desempenho do método em [11];

- O pequeno aumento percentual na Taxa Espacial de Detecção média proporcionado pelo método em [11], em relação ao método proposto, se faz ao custo de um aumento percentual bem maior na Taxa Espacial de Falso Alarme média, que se reflete numa redução não desprezível da capacidade do HDFSS;

- Os resultados obtidos indicam que quando o sinal do usuário primário tem DEP plana (amostras descorrelatadas), é possível se trabalhar com Taxas Espaciais de Falso Alarme menores (maior capacidade do HDFSS) do que as taxas mínimas obtidas quando os sinais dos UPs têm DEP de dois polos;

- Com relação ao número de enlaces FS presentes na área de serviço do HDFSS, observou-se que, embora os valores de $\bar{T}_{d}$ nos dois cenários estejam bem próximos, existe um aumento nos valores de $\bar{T}_{f a}$ do Cenário 2 (3 enlaces FS) em relação àqueles do Cenário 1 (apenas 1 enlace FS). Isto sugere que a capacidade utilizada pelo sistema HDFSS diminui com o aumento do número de usuários primários operando na região de interesse;

- No terceiro caso considerado em cada um dos cenários, foi avaliada a situação onde, embora o sinal produzido pelas transmissões dos usuários 
primários tenha uma densidade espectral de potência de dois polos, o DBC gera o Mapa de Ambiente de Rádio considerando que a densidade espectral de potência dos sinais transmitidos pelos usuários primários é plana. Observou-se, nesta situação, que a variância $\left(T_{f a}, T_{d}\right)$, (refletida no tamanho das nuvens de pontos) aumenta, indicando uma pequena queda no desempenho da geração de REM, em relação ao caso em que o DBC considera que os sinais transmitidos pelos usuários primários têm DEP de dois polos.

\section{Trabalhos Futuros}

- Analise do desempenho do método de geração de REM proposto considerando outros tipos de densidade espectral de potência para o sinal produzido pelas transmissões de usuários primários.

- Analise quantitativa mais abrangente da redução de capacidade no HDFSS resultante do aumento do número de usuários primários na área de serviço do HDFSS.

- Considerar HDFSS utilizando satélites em orbitas não-geoestacionárias

- Estudos visando a proposição de outros métodos de geração de REM, por exemplo, métodos onde a quantidade de informação enviada ao DBC é maior (lembrar que existe um compromisso entre a quantidade de informação enviada e a qualidade do REM).

- Analise do problema de compartilhamento de frequências entre o sistema HDFSS e outro tipo de serviços terrestres. 


\section{Referências bibliográficas}

[1] P. G. Moschopoulos, The distribution of the sum of independent gamma random variables, Annals of the Institute of Statistical Mathematics, vol. 37, no. 1 , pp. 541-544, 1985.

[2] S. P. T. Force, Spectrum policy task force report, FCC ET Docket, vol. 2, p. 135, 2002.

[3] E. FCC, Docket No 03-222 Notice of proposed rule making and order, 2003.

[4] D. McHenry and D. McCloskey, Spectrum occupancy report for New York city during the republican convention, 2004.

[5] M. Marcus, J. Burtle, B. Franca, A. Lahjouji, and N. McNeil, Federal communications commission spectrum policy task force, Report of the Unlicensed Devices and Experimental Licenses Working Group, 2002.

[6] M. Höyhtyä, J. Kyröläinen, A. Hulkkonen, J. Ylitalo, and A. Roivainen, Application of cognitive radio techniques to satellite communication, in Dynamic Spectrum Access Networks (DYSPAN), 2012 IEEE International Symposium on. IEEE, 2012, pp. 540-551.

[7] S. Maleki, S. Chatzinotas, B. Evans, K. Liolis, J. Grotz, A. Vanelli-Coralli, and N. Chuberre, Cognitive spectrum utilization in Ka bandmultibeam satellite communications, IEEE Communications Magazine, 2015.

[8] S. Maleki, S. Chatzinotas, J. Krause, K. Liolis, and B. Ottersten, Cognitive Zone for Broadband Satellite Communications in 17.3-17.7 GHz Band, IEEE Wireless Communications Letters, vol. 4, no. 3, pp. 305-308, 2015.

[9] S. K. Sharma, S. Chatzinotas, and B. Ottersten, Satellite cognitive communications: Interference modeling and techniques selection, in Advanced Satellite Multimedia Systems Conference (ASMS) and 12th Signal Processing for Space Communications Workshop (SPSC), 2012 6th. IEEE, 2012, pp. 111-118. 
[10] M. Höyhtyä, Frequency sharing between FSS and BSS satellites in the 17.3 18.4 GHz band, Advances in Wireless and Optical Communications (RTUWO), 2015.

[11] V. Icolari, D. Tarchi, A. Vanelli-Coralli, and M. Vincenzi, An energy detector based radio environment mapping technique for cognitive satellite systems, in GLOBECOM 2014.

[12] S. Maleki, S. Chatzinotas, S. K. Sharma, A. Guidotti, D. Tarchi, A. VanelliCoralli, W. Tang, B. G. Evans, J. Grotz, K. Liolis et al., Cognitive radio for Ka band satellite communications, in 32nd AIAA International Communications Satellite Systems Conference, 2014.

[13] W. Tang, P. Thompson, and B. Evans, Frequency sharing between satellite and terrestrial systems in the Ka band: A database approach, in ICC 2015.

[14] A. Guidotti, V. Icolari, D. Tarchi, A. Vanelli-Coralli, S. K. Sharma, E. Lagunas, S. Maleki, S. Chatzinotas, J. Grotz, J. Krause et al., Spectrum awareness and exploitation for cognitive radio satellite communications, in Networks and Communications (EuCNC), 2015 European Conference on. IEEE, 2015, pp. 16-20.

[15] S. K. Sharma, S. Maleki, S. Chatzinotas, J. Grotz, J. Krause, and B. Ottersten, Joint carrier allocation and beamforming for cognitive SatComs in Ka-band (17.3-18.1 GHz), in Communications (ICC), 2015 IEEE International Conference on. IEEE, 2015, pp. 873-878.

[16] E. Lagunas, S. K. Sharma, S. Maleki, S. Chatzinotas, and B. Ottersten, Resource allocation for cognitive satellite communications with incumbent terrestrial networks, IEEE Transactions on Cognitive Communications and Networking, vol. 1, no. 3, pp. 305-317, 2015.

[17] R. Umar and A. U. Sheikh, A comparative study of spectrum awareness techniques for cognitive radio oriented wireless networks, Physical Communication Journal 2013, vol. 9.

[18] R. Umar, A. U. Sheikh, and M. Deriche, Unveiling the hidden assumptions of energy detector based spectrum sensing for cognitive radios, IEEE Communications Surveys \& Tutorials, vol. 16, 2014.

[19] Y. Zhao, L. Morales, J. Gaeddert, K. K. Bae, J.-S. Um, and J. H. Reed, Applying radio environment maps to cognitive wireless regional area networks, in DySPAN 2007. 
[20] Guidelines for implementation of high-density applications in the fixed-satellite service in frequency bands identified for these applications, Resolution 143 (WRC-03), Geneva, 2003.

[21] Draft U.S. Proposal on WRC-03 Agenda Item 1.25, WRC-2003 Advisory Committee IWG-4, Geneva, 2001.

[22] Technical characteristics of high-density fixed-satellite service earth stations operating with geostationary satellite orbit fixed-satellite service networks in the 20/30 GHz bands, Question ITU-R 266/4, 2001.

[23] Maximum emission levels and associated requirements of high density fixedsatellite service earth stations transmitting towards geostationary fixedsatellite service space stations in the $30 \mathrm{GHz}$ range, Recommendation ITU-R S.1594, Geneva, 2002.

[24] Technical and operational features characterizing high-density applications in the fixed-satellite service, Recommendation ITU-R S.1783, Geneva, 2007.

[25] ITU, Radio Regulations 2012, Radiocommunication Sector. ITU-R.

[26] K. Miya, Satellite communications technology, 2nd ed. KDD Engineering and Consulting Inc, 1985, pp 424-425.

[27] J. Mendez R and J. M. Fortes, A detection probability based radio environment mapping technique for cognitive high density fixed satellite systems, in Computing and Communication Workshop and Conference (CCWC), 2018 IEEE 8th Annual. IEEE, 2018, pp. 779-785.

[28] F. F. Digham, M.-S. Alouini, and M. K. Simon, On the energy detection of unknown signals over fading channels, IEEE Transactions on communications, vol. 55, no. 1, pp. 21-24, 2007.

[29] H. Urkowitz, Energy detection of unknown deterministic signals, Proceedings of the IEEE, vol. 55, no. 4, pp. 523-531, 1967.

[30] V. I. Kostylev, Energy detection of a signal with random amplitude, in ICC 2002.

[31] A. Mariani, A. Giorgetti, and M. Chiani, Effects of noise power estimation on energy detection for cognitive radio applications, IEEE Transactions on Communications, vol. 59, no. 12, pp. 3410-3420, 2011.

[32] J. D. Gibson, Mobile communications handbook, 3rd ed. CRC press, 2013. 
[33] J. Mendez Rangel and J. M. Fortes, Mapa de Ambiente de Rádio baseado na Probabilidade de Detecção para uso em Sistemas Cognitivos de Alta densidade via Satélite, Simposio Brasileiro de Telecomunicações e Processamento de Sinais - SBrT2018, pp. 2892-2897, Setembro 2018.

[34] A. Mariani, A. Giorgetti, and M. Chiani, Energy detector design for cognitive radio applications, in WDD Conference, 2010.

[35] D. Torrieri, Principles of spread-spectrum communication systems, 2nd ed. Springer, 2005.

[36] R. G. Gallager, Stochastic processes: theory for applications. Cambridge University Press, 2013.

[37] D. Tse and P. Viswanath, Fundamentals of Wireless Communication. Cambridge University Press, 2005.

[38] F. F. Digham, M.-S. Alouini, and M. K. Simon, On the energy detection of unknown signals over fading channels, in ICC 2003, vol. 5 . leee, 2003, pp. 3575-3579.

[39] A. Ghasemi and E. S. Sousa, Collaborative spectrum sensing for opportunistic access in fading environments, in DySPAN 2005.

[40] Z. Ye, G. Memik, and J. Grosspietsch, Energy detection using estimated noise variance for spectrum sensing in cognitive radio networks, in WCNC 2008.

[41] K. Arshad, M. A. Imran, and K. Moessner, Collaborative spectrum sensing optimisation algorithms for cognitive radio networks, International Journal of Digital Multimedia Broadcasting, 2010.

[42] H. Wang, X. Su, Y. Xu, S. Zhou, and J. Wang, Optimal cooperative energy spectrum sensing in cognitive radio network, Frontiers of Electrical and Electronic Engineering Journal, vol. 5, 2010.

[43] J. P. d. A. Albuquerque, J. M. P. Fortes, and W. A. Finamore, Probabilidade, variáveis aleatórias e processos estocásticos, Editora PUC-Rio e Editora Interciência, Rio de Janeiro, Brasil, 2008.

[44] L. A. Aguirre, Introdução à identificação de sistemas-Técnicas lineares e nãolineares aplicadas a sistemas reais. Editora UFMG, 2004.

[45] I. S. Gradshteyn and I. M. Ryzhik, Table of integrals, series, and products. Academic press, 1965. 
[46] F. Aurenhammer, R. Klein, and D.-T. Lee, Voronoi diagrams and Delaunay triangulations. World Scientific Publishing Company, 2013.

[47] H. L. Van Trees, Detection, estimation and modulation theory, part l:, detection, estimation and linear modulation theory. Wiley, 1968.

[48] Reference radiation patterns for fixed wireless system antennas for use in coordination studies and interference assessment in the frequency range from $100 \mathrm{MHz}$ to about $86 \mathrm{GHz}$, Recommendation ITU-R F.699, Geneva, 2018.

[49] S. N. Chiu, D. Stoyan, W. S. Kendall, and J. Mecke, Stochastic geometry and its applications. John Wiley \& Sons, 2013.

[50] System parameters and considerations in the development of criteria for sharing or compatibility between digital fixed wireless systems in the fixed service and systems in other services and other sources of interference, Recommendation ITU-R F.758, Geneva, 2015. 
A

\section{Soma de variáveis aleatórias com função densidade de proba- bilidade exponencial e estatisticamente independentes}

As seções a seguir resumem dois dos procedimentos passiveis de serem utilizados na determinação da função densidade de probabilidade da soma de variáveis aleatórias com função densidade de probabilidade exponencial e estatisticamente independentes. Em outras palavras o problema consiste em determinar a função densidade de probabilidade da variável aleatória

$$
v=\sum_{i=1}^{M} y_{i}
$$

no caso em que as variáveis aleatórias $\left\{y_{i}, i=1, \ldots, M\right\}$ são estatisticamente independentes, com função densidade de probabilidade dada por

$$
p_{y_{i}}(Y)=c_{i} e^{-c_{i} Y} u(Y) \quad ; \quad c_{i}>0
$$

com $u(Y)$ denotando a função degrau unitário definida em (3-108).

A função caraterística das variáveis aleatórias $\left\{y_{i}, i=1, \ldots, M\right\}$, com função densidade de probabilidade exponencial é dada

$$
\mathcal{M}_{y_{i}}(\nu)=E\left[e^{j \nu y_{i}}\right]=\frac{c_{i}}{c_{i}-j \nu} \quad ; \quad i=1,2, \ldots, M
$$

(com $j$ representando a raíz quadrada de -1 ), como as variáveis aleatórias $\left\{y_{i}, i=1, \ldots, M\right\}$ são estatisticamente independentes, a função caraterística da variável aleatória $v$ em (A-1) é dada por

$$
\mathcal{M}_{v}(\nu)=\prod_{i=1}^{M} \mathcal{M}_{y_{i}}(\nu)=\prod_{i=1}^{M} \frac{c_{i}}{c_{i}-j \nu}
$$

A função densidade de probabilidade da variável aleatória $v$ é então dada pela integral

$$
p_{v}(V)=\frac{1}{2 \pi} \int_{-\infty}^{\infty} \mathcal{M}_{v}(\nu) e^{-j \nu V} d \nu
$$

com $c_{i}$ dado por (3-107).

\section{A.1}

\section{Procedimento 1: utilizando o método dos resíduos}

A integral em (A-5) pode ser calculada por resíduos sendo, em geral dada por 
Apêndice A. Soma de variáveis aleatórias com função densidade de

$$
p_{v}(V)=\left(-\sum_{\ell=1}^{L} r_{\ell}\right) u(V),
$$

onde $L$ é o número de singularidades da função

$$
f(x)=\left(\prod_{i=1}^{M} \frac{c_{i}}{c_{i}-x}\right) e^{-x V},
$$

no semiplano direito e $r_{\ell}$ é o resíduo desta função na $\ell$-ésima singularidade. Se a função tem um polo de ordem $m$ em $x_{0}$, correspondente à $\ell$-ésima singularidade, tem-se

$$
r_{\ell}=\frac{1}{(m-1) !} \lim _{x \rightarrow x_{0}} \frac{d^{m-1}}{d x^{m-1}}\left(x-x_{0}\right)^{m} f(x) .
$$

No caso particular em que a função $f(x)$ tem apenas $L$ polos de primeira ordem em $\left\{c_{i}, i=1, \ldots, M\right\}$, então $L=M$ e $p_{v}(V)$ se escreve

$$
p_{v}(V)=\sum_{\ell=1}^{M} A_{\ell} e^{-c_{\ell} V}
$$

onde

$$
A_{\ell}=\left(\prod_{i=1}^{M} c_{i}\right)\left(\prod_{\substack{i=1 \\ i \neq \ell}}^{M} c_{i}-c_{\ell}\right)^{-1}
$$

\section{A. 2}

Procedimento 2: utilizando um caso particular da série convergente proposta em [1]

Conforme indicado em [1], a integral em (A-5) pode, alternativamente, ser calculada utilizando-se a série convergente definida em [1]. O caso particular da série para este trabalho é dada por

$$
p_{v}(V)=C \sum_{k=0}^{\infty} \delta_{k} \frac{V^{M+k-1}\left(c^{*}\right)^{M+k}}{\Gamma(M+k)} \exp \left(-c^{*} V\right) u(V)
$$

onde

$$
c^{*}=\max _{i}^{-1} c_{i}
$$

$\mathrm{e}$

$$
C=\prod_{i=1}^{M} \frac{c_{i}}{c^{*}} .
$$

Em (A-11), os coeficientes $\delta_{k}$ são dados por

$$
\delta_{k}= \begin{cases}1 & ; k=0 \\ \frac{1}{k} \sum_{i=1}^{k} i \gamma_{i} \delta_{k-i} & ; k=1,2, \ldots\end{cases}
$$

onde

$$
\gamma_{k}=\sum_{i=1}^{M} \frac{\left(1-c_{i} / c^{*}\right)^{k}}{k} ; \quad k=1,2,3 \ldots
$$


B

\section{Estimadores utilizados pelos USs e no DBC}

\section{B.1}

Estimador com base na observação da variável de decisão $v$ (sinais de UPs com densidade espectral de potência genérica)

Nesta Seção, é obtida a Equação a ser satisfeita pelo estimador subótimo da potência média $\beta$ da envoltória complexa do sinal interferente produzido pelas transmissões dos usuários primários quando este sinal é caracterizado por um processo estocástico passa-faixa gaussiano ESA de média nula com densidade espectral de potência genérica e conhecida. Considera-se que esta estimativa é feita com base no valor assumido pela variável de decisão $v$ definida em (3-93), sendo, neste caso, dada por (3-148), ou seja,

$$
\hat{\beta}_{1}(V)=\max _{B}^{-1}\left[C \sum_{k=0}^{\infty} \delta_{k} \frac{V^{M+k-1} \exp \left(-\frac{V}{2 \sigma_{n}^{2} B \lambda^{*}}\right)}{\Gamma(M+k)\left(2 \sigma_{n}^{2} B \lambda^{*}\right)^{M+k}} u(V)\right]
$$

Note que (B-1) pode ainda ser escrita como

$$
\hat{\beta}_{1}(V)=\max _{B}^{-1}\left[A(V) \exp \left(-\frac{V}{2 \sigma_{n}^{2} B \lambda^{*}}\right) \sum_{k=0}^{\infty} M_{k}(V) \frac{1}{B^{M+k}} u(V)\right]
$$

onde

$$
\begin{gathered}
A(V)=\frac{C V^{M-1}}{\left(2 \sigma_{n}^{2} \lambda^{*}\right)^{M}} \\
M_{k}(V)=\frac{\delta_{k} V^{k}}{\Gamma(M+k)\left(2 \sigma_{n}^{2} \lambda^{*}\right)^{k}}
\end{gathered}
$$

A Equação, a ser resolvida para a obtenção de $\hat{\beta}_{1}(V)$ é então obtida considerando-se a condição

$$
\frac{\partial}{\partial B}\left[A(V) \exp \left(-\frac{V}{2 \sigma_{n}^{2} B \lambda^{*}}\right) \sum_{k=0}^{\infty} M_{k}(V) \frac{1}{B^{M+k}}\right]=0 \quad ; \quad V \geq 0
$$

Efetuando-se a derivada em (B-5), obtém-se,

$$
\begin{aligned}
A(V) & {\left[\exp \left(-\frac{V}{2 \sigma_{n}^{2} B \lambda^{*}}\right) \frac{V}{2 \sigma_{n}^{2} \lambda^{*}} \frac{1}{B^{2}} \sum_{k=0}^{\infty} M_{k}(V) \frac{1}{B^{M+k}}+\right.} \\
& \left.\exp \left(-\frac{V}{2 \sigma_{n}^{2} B \lambda^{*}}\right) \sum_{k=0}^{\infty} M_{k}(V)(M+k)\left(\frac{1}{B}\right)^{M+k-1}\left(-\frac{1}{B^{2}}\right)\right]=0
\end{aligned}
$$


ou ainda,

$$
A(V) \exp \left(-\frac{V}{2 \sigma_{n}^{2} B \lambda^{*}}\right) \frac{1}{B^{M+2}}\left[\frac{V}{2 \sigma_{n}^{2} \lambda^{*}} \sum_{k=0}^{\infty} \frac{M_{k}(V)}{B^{k}}-\sum_{k=0}^{\infty} \frac{M_{k}(V)}{B^{k-1}}(M+k)\right]=0 .
$$

Considerando-se que

$$
A(V) \exp \left(-\frac{V}{2 \sigma_{n}^{2} B \lambda^{*}}\right) \frac{1}{B^{M+2}} \neq 0
$$

obtém-se finalmente a equação

$$
\sum_{k=0}^{\infty} \frac{M_{k}(V)}{B^{k-1}}\left(\frac{V}{2 \sigma_{n}^{2} \lambda^{*} B}-M\right)-\sum_{k=0}^{\infty} k \frac{M_{k}(V)}{B^{k-1}}=0,
$$

que deve ser resolvida em relação a $B$. Observe que, nas situações em que a segunda parcela da expressão acima é desprezível relativamente à primeira parcela, a Equação tem uma solução fechada, dada por,

$$
B=\frac{V}{M 2 \sigma_{n}^{2} \lambda^{*}} \text {. }
$$

Entretanto, testes feitos a partir de resultados numéricos indicaram que esta premissa não é verdadeira na grande maioria dos casos. Assim, a solução de (B-9) tem que ser obtida numericamente, o que pode requerer algoritmos complexos (note que (B-9) tem múltiplas raízes).

\section{B.2}

\section{Estimador com base na observação da variável de decisão $v$ (sinais de UPs com densidade espectral de potência plana)}

Nesta Seção, é obtida a Equação a ser satisfeita pelo estimador subótimo da potência média $\beta$ da envoltória complexa do sinal interferente produzido pelas transmissões dos usuários primários quando este sinal é caracterizado por um processo estocástico passa-faixa gaussiano ESA de média nula com densidade espectral de potência plana. Considera-se que esta estimativa é feita com base no valor assumido pela variável de decisão $v$ definida em (3-163), sendo, neste caso, dada por (3-192), ou seja,

$$
\hat{\beta}_{1}(V)=\max _{B}^{-1}\left[\frac{1}{\left(2 \sigma_{n}^{2} B\right)^{M}} \frac{V^{M-1}}{\Gamma(M)} \exp \left(-\frac{V}{2 \sigma_{n}^{2} B}\right) u(V)\right]
$$

Note que (B-11) pode ainda ser escrita como

$$
\hat{\beta}_{1}(V)=\max _{B}^{-1}\left[K \exp \left(-\frac{V}{2 \sigma_{n}^{2} B}\right)\left(\frac{1}{B}\right)^{M} u(V)\right]
$$

onde

$$
K=\frac{1}{\left(2 \sigma_{n}^{2}\right)^{M}} \frac{V^{M-1}}{\Gamma(M)}
$$


A equação, a ser resolvida para a obtenção de $\hat{\beta}_{1}(V)$ é então obtida considerando-se a condição

$$
\frac{\partial}{\partial B}\left[K \exp \left(-\frac{V}{2 \sigma_{n}^{2} B}\right)\left(\frac{1}{B}\right)^{M} u(V)\right]=0 \quad ; \quad V \geq 0
$$

Efetuando-se a derivada em (B-14), obtém-se,

$K\left[\exp \left(-\frac{V}{2 \sigma_{n}^{2} B}\right) \frac{V}{2 \sigma_{n}^{2}} \frac{1}{B^{2}}\left(\frac{1}{B}\right)^{M}+\exp \left(-\frac{V}{2 \sigma_{n}^{2} B}\right) M\left(\frac{1}{B}\right)^{M-1}\left(-\frac{1}{B^{2}}\right)\right]=0$

ou ainda,

$$
K \exp \left(-\frac{V}{2 \sigma_{n}^{2} B}\right) \frac{1}{B^{2} B^{M-1}}\left[\frac{1}{B} \frac{V}{2 \sigma_{n}^{2}}-M\right]=0
$$

Considerando-se que

$$
K \exp \left(-\frac{V}{2 \sigma_{n}^{2} B}\right) \frac{1}{B^{2} B^{M-1}} \neq 0
$$

obtém-se a equação

$$
\frac{1}{B} \frac{V}{2 \sigma_{n}^{2}}-M=0
$$

que deve ser resolvida em relação a $B$. Assim a Equação tem uma solução fechada, dada por

$$
\hat{\beta}_{1}(V)=\frac{V}{M 2 \sigma_{n}^{2}} .
$$

\section{B.3}

\section{Utilização do estimador de potência para sinais com DEP plana em situações onde a DEP dos sinais é não plana}

\section{B.3.1}

\section{Estimação feita nos usuários secundários}

Para avaliar a qualidade do estimador subótimo definido em (3-190) em situações onde o sinal dos UPs tem DEP genérica, as curvas do tipo ROC (taxa espacial de detecção versus taxa espacial de falso alarme) obtidas utilizandose este estimador foram comparadas àquelas obtidas com o valor real do parâmetro $\beta$ (disponível na simulação). Visando quantificar esta comparação são definidos dois parâmetros: a variação relativa da taxa espacial média de detecção e a variação relativa da taxa espacial média de falso alarme. Estes parâmetros são definidos respectivamente por

$$
\Delta \bar{T}_{d}=\frac{\bar{T}_{d_{\hat{\beta}}}-\bar{T}_{d_{\beta}}}{\bar{T}_{d_{\beta}}}
$$

e

$$
\Delta \bar{T}_{f a}=\frac{\bar{T}_{f a_{\hat{\beta}}}-\bar{T}_{f a_{\beta}}}{\bar{T}_{f a_{\beta}}}
$$


onde $\bar{T}_{d_{\beta}}$ e $\bar{T}_{f a_{\beta}}$ denotam as taxas espaciais médias de detecção e de falso alarme obtidas ao se utilizar o valor real do parâmetro $\beta$, e $\bar{T}_{d_{\hat{\beta}}}$ e $\bar{T}_{f a_{\hat{\beta}}}$ denotam as taxas espacias médias de detecção e de falso alarme obtidas utilizando-se a estimativa $\hat{\beta}$ em (3-190). Note que estas últimas taxas espaciais médias são indicadas por asteríscos nas figuras 5.10 a $5.12,5.14$ a 5.16, 5.18 a 5.20, 5.25 a $5.27,5.29$ a 5.31 e 5.33 a 5.35 .

Em cada um dos cenários e casos abordados na Seção 5.4, os valores de $\Delta \bar{T}_{d}$ e $\Delta \bar{T}_{f a}$ foram calculados para cada um dos quatro níveis de quantização considerados: $\ell=0,5, \ell=0,6, \ell=0,7 \mathrm{e} \ell=0,8$. As médias aritméticas $\Delta_{d}$ (dos 4 valores de $\left.\Delta \bar{T}_{d}\right)$ e $\Delta_{f a}\left(\operatorname{dos} 4\right.$ valores de $\left.\Delta \bar{T}_{f a}\right)$ obtidas são apresentadas na Tabela B.1.

Tabela B.1: Média aritmética dos valores de $\Delta \bar{T}_{d}$ e $\Delta \bar{T}_{f a}$ para cada um dos casos e cenários abordados na Seção 5.4

\begin{tabular}{|l|c|c|c|c|c|c|c|}
\cline { 3 - 8 } \multicolumn{2}{c|}{} & \multicolumn{2}{c|}{450 USs } & \multicolumn{2}{c|}{900 USs } & \multicolumn{2}{c|}{1350 USs } \\
\cline { 3 - 8 } \multicolumn{2}{c|}{} & $\Delta_{d}$ & $\Delta_{f a}$ & $\Delta_{d}$ & $\Delta_{f a}$ & $\Delta_{d}$ & $\Delta_{f a}$ \\
\hline \multirow{3}{*}{ Cenário 1 } & Caso 1 & 0,000134 & 0,242752 & $-0,00636$ & 0,175006 & $-0,00496$ & 0,144646 \\
\cline { 2 - 8 } & Caso 2 & 0,004347 & 0,093593 & $-0,00281$ & 0,076514 & $-0,00386$ & 0,064347 \\
\cline { 2 - 8 } & Caso 3 & 0,007019 & 0,10073 & $-0,00274$ & 0,07689 & $-0,00414$ & 0,064131 \\
\hline \multirow{3}{*}{ Cenário 2 } & Caso 1 & $-0,00797$ & 0,12101 & $-0,00525$ & 0,08128 & $-0,00363$ & 0,06167 \\
\cline { 2 - 8 } & Caso 2 & $-0,00147$ & 0,07600 & $-0,00388$ & 0,05905 & $-0,00411$ & 0,04760 \\
\cline { 2 - 8 } & Caso 3 & $-0,00111$ & 0,07646 & $-0,00379$ & 0,05973 & $-0,00412$ & 0,04709 \\
\hline
\end{tabular}

Observe que, de uma maneira geral, as médias aritméticas das variações relativas das taxas espaciais médias de detecção ficaram, em módulo, abaixo de 0,01 e as médias aritméticas das variações relativas das taxas espaciais médias de falso alarme ficaram, em módulo, abaixo de 0,1. Apenas no Cenário 1 , Caso 1 , observou-se valores de $\Delta_{f a}$ mais significativos, da ordem de 0,242 (450 USs), 0,175 (900 USs) e 0,144 (1350 USs). Mesmo assim, observe que, em praticamente todos os casos, a utilização do estimador em (3-190) produziu resultados um pouco conservadores (menores valores de $\bar{T}_{d_{\hat{\beta}}}$ e maiores valores de $\bar{T}_{f a_{\hat{\beta}}}$, quando comparados a $\bar{T}_{d_{\beta}}$ e $\bar{T}_{f a_{\beta}}$, respectivamente).

Note ainda que, conforme esperado, os valores de $\Delta_{d}$ e $\Delta_{f a}$ diminuem à medida em que o número de USs cooperativas aumenta. Os resultados apresentados na Tabela B.1 ilustram o pequeno efeito causado pela utilização do estimador subótimo em (3-190) no processamento feito pelos usuários secundários, justificando a sua utilização no método de geração de REM proposto. 


\section{B.3.2}

\section{Estimação feita no Centro de Base de Dados}

Nesta seção, é analisado o efeito de utilizar o estimador em (B-19), definido para o caso em que o sinal interferente produzido pelas transmissões de usuários primários tem densidade espectral de potência plana em situações em que a densidade espectral de potência tem dois polos (ver 5.1).

Inicialmente foi considerado que o nível de potência da envoltória complexa do sinal produzido pelas transmissões de usuários primários assume um valor $\beta=B \mathrm{~dB}(\mathrm{~W} / \mathrm{MHz})$, que varia em um intervalo de $B=$ [-190 dB(W/MHz) : $-100 \mathrm{~dB}(\mathrm{~W} / \mathrm{MHz})]$. Assim, foram considerados 10 valores dentro desse intervalo e para cada um desses valores foram geradas $N=10000$ amostras $\left\{V_{j}, j=1, \ldots, N\right\}$ da variável decisão $v$ dada por (3-93), a partir destes valores e utilizando a expressão em (B-19), foram obti$\operatorname{dos} N=10000$ valores para a estimativa do parâmetro $\left\{\hat{\beta}_{j_{d B}}, j=1, \ldots, N\right\}$, implicando em 10000 valores do erro de estimação dado por

$$
e_{j_{d B}}=\hat{\beta}_{j_{d B}}-B_{d B} \quad ; \quad j=1, \ldots, N
$$

Uma análise estatística dos $N$ valores do erro de estimação obtidos para cada valor definido $\beta=B \mathrm{~dB}(\mathrm{~W} / \mathrm{MHz})$ conduziu aos resultados apresentados na tabela B.2.

Tabela B.2: Estatísticas do erro de estimação para vários valores de $\beta=B$ $\mathrm{dB}(\mathrm{W} / \mathrm{MHz})$

\begin{tabular}{|c|c|c|c|c|c|c|}
\hline$B_{d B}$ & $m_{e_{\mathrm{dB}}}$ & $\sigma_{e_{\mathrm{dB}}}^{2}$ & $\sigma_{e_{\mathrm{dB}}}$ & $P\left(\left|e_{j_{\mathrm{dB}}}\right|<0,1\right)$ & $P\left(\left|e_{j_{\mathrm{dB}}}\right|<0,5\right)$ & $P\left(\left|e_{j_{\mathrm{dB}}}\right|<1\right)$ \\
\hline-190 & $-0,0022$ & 0,0774 & 0,2782 & 0,2786 & 0,9280 & 0,9998 \\
\hline-180 & $-0,0076$ & 0,0767 & 0,2770 & 0,2857 & 0,9312 & 0,9995 \\
\hline-170 & $-0,0095$ & 0,0787 & 0,2806 & 0,2819 & 0,9253 & 0,9995 \\
\hline-160 & $-0,0093$ & 0,0806 & 0,2839 & 0,2756 & 0,9213 & 0,9997 \\
\hline-150 & $-0,0090$ & 0,0771 & 0,2777 & 0,2744 & 0,9300 & 0,9995 \\
\hline-140 & $-0,0085$ & 0,0775 & 0,2784 & 0,2813 & 0,9280 & 0,9998 \\
\hline-130 & $-0,0068$ & 0,0761 & 0,2759 & 0,2827 & 0,9322 & 0,9995 \\
\hline-120 & $-0,0081$ & 0,0767 & 0,2769 & 0,2785 & 0,9298 & 0,9997 \\
\hline-110 & $-0,0051$ & 0,0771 & 0,2776 & 0,2875 & 0,9284 & 0,9996 \\
\hline-100 & $-0,0137$ & 0,0784 & 0,2800 & 0,2792 & 0,9255 & 1,0000 \\
\hline
\end{tabular}

Na tabela B.2, $m_{e_{\mathrm{dB}}}$ representa a média do erro de estimação dada por

$$
m_{e_{\mathrm{dB}}}=\frac{1}{N} \sum_{j=1}^{N} e_{j_{\mathrm{dB}}},
$$

$\sigma_{e_{\mathrm{dB}}}^{2}$ representa a variância do erro de estimação dada por 


$$
\sigma_{e_{\mathrm{dB}}}^{2}=\frac{1}{N} \sum_{j=1}^{N}\left(e_{j_{\mathrm{dB}}}-m_{e_{\mathrm{dB}}}\right)^{2}
$$

e $\sigma_{e_{\mathrm{dB}}}$ representa o desvio padrão, dado por

$$
\sigma_{e_{\mathrm{dB}}}=\sqrt{\sigma_{e_{\mathrm{dB}}}^{2}}
$$

A partir dos resultados na Tabela B.2 observou-se que a razão $\left|\frac{\sigma_{e_{\mathrm{dB}}}}{B_{d B}}\right|$ pertinente ao erro de estimação é muito pequena. A probabilidade do valor absoluto do erro ser menor a $0,5 \mathrm{~dB}$ é maior que 0,9 e a probabilidade de do valor absoluto do erro ser menor que $1 \mathrm{~dB}$ tende a 1 . Assim estes resultados validam o uso do estimador em (B-19) no caso em que o sinal interferente produzido pelas transmissões de usuários primários tem densidade espectral de potência com dois polos. Portanto, o resultado de esta análise foi considerado no desenvolvimento do Capítulo 5. 